\title{
Updated checklist of marine fishes (Chordata: Craniata) from Portugal and the proposed extension of the Portuguese continental shelf
}

\author{
Miguel CARNEIRO ${ }^{1,5}$, Rogélia MARTINS ${ }^{2,6}$, Monica LANDI ${ }^{*}, 3,7$ \& Filipe O. COSTA ${ }^{4,8}$ \\ ${ }^{1,2}$ DIV-RP (Modelling and Management Fishery Resources Division), Instituto Português do Mar \\ e da Atmosfera, Av. Brasilia 1449-006 Lisboa, Portugal. \\ E-mail: mcarneiro@,ipma.pt, rmartins@ipma.pt \\ ${ }^{3,4}$ CBMA (Centre of Molecular and Environmental Biology), Department of Biology, \\ University of Minho, Campus de Gualtar, 4710-057 Braga, Portugal. \\ E-mail: mlandi@bio.uminho.pt, fcosta@bio.uminho.pt \\ *corresponding author: mlandi@bio.uminho.pt \\ ${ }^{5}$ urn:1sid:zoobank.org:author:90A98A50-327E-4648-9DCE-75709C7A2472 \\ ${ }^{6}$ urn:lsid:zoobank.org:author:1EB6DE00-9E91-407C-B7C4-34F31F29FD88 \\ ${ }^{7}$ urn:1sid:zoobank.org:author:6D3AC760-77F2-4CFA-B5C7-665CB07F4CEB \\ ${ }^{8}$ urn:1sid:zoobank.org:author:48E53CF3-71C8-403C-BECD-10B20B3C15B4
}

\begin{abstract}
The study of the Portuguese marine ichthyofauna has a long historical tradition, rooted back in the $18^{\text {th }}$ Century. Here we present an annotated checklist of the marine fishes from Portuguese waters, including the area encompassed by the proposed extension of the Portuguese continental shelf and the Economic Exclusive Zone (EEZ). The list is based on historical literature records and taxon occurrence data obtained from natural history collections, together with new revisions and occurrences. It comprises a total of 1191 species, distributed among 3 superclasses, 4 classes, 42 orders, 212 families and 617 genera. If considering only the EEZ and present territorial waters, this list represents an increase of 230 species $(27.8 \%)$ and of 238 species $(29.0 \%)$, when compared to the information available in FishBase (2012) and in the last checklist of marine and estuarine fishes of Portugal (1993), respectively. The order Perciformes shows the highest diversity, with 54 families, 162 genera and 299 species. Stomiidae (80 species), Myctophidae (71 species) and Macrouridae (37 species) are the richest families. From the listed species, 734 are present off mainland Portugal, 857 off the Azores and 766 off Madeira. Within the limits of the examined area, three species are reported for the first time in mainland Portugal and twenty-nine records are identified as doubtful. A total of 133 species have been recorded from the extended Portuguese continental shelf ( 2 off mainland Portugal, 117 off the Azores and 14 off Madeira), two of which are common to the Azores and Madeira extensions. Biogeographically, the Atlantic group is the most important ( 548 species $-46.01 \%$ ), followed by the Lusitanian group ( 256 species $-21.49 \%$ ), the African group ( 71 species $-5.96 \%$ ), the Boreal group (34 species - 2.85\%), the Mediterranean group (31 species $-2.60 \%$ ), the Macaronesian group (21 species - 1.76\%), the Atlantic/African group (19 species - 1.60\%) and the Mediterranean/African and the Arctic groups, each with only 1 species $(0.08 \%)$. Regarding the preferences for vertical habitat, the demersal fishes are the most important group (305 species $-25.61 \%$ ), followed by the mesopelagic group (228 species - 19.14\%), the bathypelagic group (164 species - 13.77\%), the benthopelagic group (147 species - 12.34\%), the bathydemersal group (115 species $-9.66 \%$ ), the reef-associated group ( 88 species $-7.39 \%)$, the pelagic group (74
\end{abstract}


species $-6.21 \%)$, the epipelagic group (58 species $-4.87 \%)$ and 1 species $(0.08 \%)$ of the benthic group. The oceanic habitat is the best represented group comprising 446 species $(37.45 \%)$, followed by the shelf group (199 species - 16.71\%), the slope group (164 species - 13.77\%), the inner shelf group (89 species $-7.47 \%)$, the coastal group ( 70 species $-5.88 \%)$, the outer shelf group $(29$ species $-2.43 \%)$ and the oceanic/shelf group ( 7 species $-0.59 \%$ ).

Key words. North East Atlantic, ichthyofauna, biodiversity, biogeography, Economic Exclusive Zone.

Carneiro M., Martins R., Landi M. \& Costa F.O. 2014. Updated checklist of marine fishes (Chordata: Craniata) from Portugal and the proposed extension of the Portuguese continental shelf. European Journal of Taxonomy 73: 1-73. http://dx.doi.org/10.5852/ejt.2014.73

\section{Introduction}

Portugal has the third largest Exclusive Economic Zone (EEZ) of the EU and the twentieth largest EEZ in the world, with a total of $1.727 .408 \mathrm{~km}^{2}$ divided between mainland Portugal $\left(327.667 \mathrm{~km}^{2}\right)$, the Archipelago of the Azores $\left(953.633 \mathrm{~km}^{2}\right.$ ) and the Archipelago of Madeira (446.108 $\left.\mathrm{km}^{2}\right)$. In 2005, a task group was created with the mission to prepare a proposal for the extension of the EEZ beyond 200 nautical miles, to be presented to the United Nations Commission on the Limits of the Continental Shelf (CLCS). With this proposal, over $2.500 .000 \mathrm{~km}^{2}$ may potentially be added to the EEZ, therefore amounting to a total Portuguese area of $4.227 .408 \mathrm{~km}^{2}$.

The Portuguese EEZ includes a variety of geographic zones. The northeastern Atlantic Ocean may be divided into two main biogeographic regions: the Lusitanian (west of the British Isles, Bay of Biscay, Iberian coast as far as Gibraltar) and northern European seas (including North Sea and Baltic Sea). The Portuguese mainland, with a coastline of approximately $850 \mathrm{~km}$, is included in the Lusitanian biogeographical region. A clearly distinct area is also the Macaronesian Islands region. The Macaronesian biogeographical region includes volcanic islands in the Atlantic Ocean, namely the Archipelago of the Azores, the Archipelago of Madeira, the Canary Islands and the Cabo Verde Islands.

The variety of ecosystems in this vast area and the geographic position of Portugal determine and affect the abundance and distribution of marine organisms. As a result, Portugal's EEZ is a dynamic biodiversity hotspot, where marine fishes from many different adjacent source areas converge (Costa et al. 2012). The marine icthyofauna of Portugal is probably one of the richest among all European countries and it appears particularly well suited to monitor changes in the marine fish species composition. Annotated species checklists constitute invaluable tools to help taking snapshots of the biodiversity characterizing a geographic area and assessing its spatial and temporal dynamics.

The annotation of the Portuguese marine ichthyofauna has a long historical tradition, rooted back to 1771, when the first checklist was compiled (Table 1). The expeditions carried out during the $18^{\text {th }}$ and $19^{\text {th }}$ centuries represented a unique opportunity for compiling new and updated lists, and since then the number of species recorded has increased.

Here we provide an updated checklist of the Portuguese ichthyofauna, including the area of the proposed extension of the Portuguese continental shelf, which builds upon the two most comprehensive checklists of marine fishes from Portugal, published in 1954 and 1993 (Albuquerque 1954-1956; Magalhães \& Rogado 1993). We also integrate data and accessory information aquired from later checklists with a regional scope, namely for the archipelagos of the Azores and Madeira. This checklist presents information as correctly and exhaustive as possible, although possible errors cannot be excluded. The authors cannot be made responsible for any errors, any misuse of data or any erroneous information reported in the citations. 
Table 1. Annotated list of historical publications reporting on marine fish species from Portugal.

\begin{tabular}{|c|c|c|c|}
\hline Date & N. species & Comment & Source \\
\hline 1771 & 60 & $\begin{array}{l}\text { Domenico Vandelli, naturalist and chemist, } \\
\text { published an elementary fish list. }\end{array}$ & Vandelli (1771) \\
\hline 1797 & 83 & $\begin{array}{l}\text { Domenico Vandelli published an updated list of } \\
\text { fish species. }\end{array}$ & Vandelli (1797) \\
\hline 1850 & 79 & $\begin{array}{l}\text { Charles Bonnet, a mining engineer, published a } \\
\text { book which included a list of fish species. }\end{array}$ & Bonnet (1850) \\
\hline 1867 & 84 & $\begin{array}{l}\text { The naturalist Felix de Brito Capello published } \\
\text { his first fish list. }\end{array}$ & Capello (1867a) (1867b) \\
\hline $1868-1876$ & 254 & $\begin{array}{l}\text { During these years Cappello added several } \\
\text { appendices to the list. }\end{array}$ & $\begin{array}{l}\text { Capello (1868) (1869a) (1869b) } \\
(1873)(1876)\end{array}$ \\
\hline 1880 & 267 & $\begin{array}{l}\text { The naturalist J.V. Barbosa du Bocage concluded } \\
\text { and published, posthumously, the work of his } \\
\text { colleague and friend F. B. Capello. }\end{array}$ & Capello (1880) \\
\hline $1888-1909$ & 209 & $\begin{array}{l}\text { Balthazar Osório published appendices and } \\
\text { addenda of } 209 \text { species to the "Catálogo dos } \\
\text { peixes de Portugal" of F. B. Capello. }\end{array}$ & $\begin{array}{l}\text { Osório (1888) (1895) (1896) } \\
(1905)(1909)\end{array}$ \\
\hline 1888 & 316 & $\begin{array}{l}\text { The publication "Éxpeditions scientifiques du } \\
\text { Travailleur et du Talisman pendant les années } \\
\text { 1880, 1881, 1882, } 1883 \text { - Poissons" included } \\
\text { a list with fish captured off the Portuguese } \\
\text { mainland and Madeira. }\end{array}$ & $\begin{array}{l}\text { Vaillant \& Milne-Edwards } \\
\text { (1880) }\end{array}$ \\
\hline $1885-1915$ & 197 & $\begin{array}{l}\text { During } 29 \text { oceanographic campaigns directed } \\
\text { by Prince Albert I of Monaco, mostly in the } \\
\text { northeastern Atlantic (Azores), } 197 \text { fish species } \\
\text { were annotated. }\end{array}$ & Porteiro (2009) \\
\hline 1896-1906 & 306 & $\begin{array}{l}\text { D. Carlos de Bragança carried out oceanographic } \\
\text { campaigns along the Atlantic Portuguese coast. } \\
\text { From these campaigns several fish collections } \\
\text { were obtained, including } 2700 \text { specimens. } \\
\text { A. A. Girard, with the collaboration of the } \\
\text { King, organized and prepared the results } \\
\text { for publication. In 1941, the naturalist B. C. } \\
\text { Gonçalves published "Colecção Oceanográfica } \\
\text { de D. Carlos I-Catálogo dos Peixes". }\end{array}$ & Gonçalves (1941) \\
\hline 1911 & 340 & $\begin{array}{l}\text { The naturalist A. F. Seabra edited and published } \\
\text { "Catalogue Systématique des Vertébrés du } \\
\text { Portugal-Poissons". }\end{array}$ & Seabra (1911) \\
\hline 1935 & 324 & $\begin{array}{l}\text { The naturalist A. Nobre published "Fauna } \\
\text { Marinha de Portugal". }\end{array}$ & Nobre (1935) \\
\hline 1954-1956 & 674 & $\begin{array}{l}\text { R. M. Albuquerque published "Peixes de } \\
\text { Portugal e Ilhas Adjacentes-Chaves para a sua } \\
\text { determinação", including } 655 \text { marine species. }\end{array}$ & Albuquerque (1954-1956) \\
\hline 1948-1971 & 388 & $\begin{array}{l}\text { During these years G. E. Maul published } \\
\text { several marine fish monographs "Monografias } \\
\text { dos peixes do Museu Municipal do Funchal" } \\
\text { (Madeira). }\end{array}$ & $\begin{array}{l}\text { Maul (1948a) (1948b) }(1948 c) \\
(1949)(1951 a)(1951 b)(1952 a) \\
(1952 b)(1954 a)(1954 b)(1955) \\
(1956 a)(1956 b)(1957)(1959) \\
(1961)(1962 a)(1962 b)(1965) \\
(1971 a)(1971 b)\end{array}$ \\
\hline
\end{tabular}




\begin{tabular}{|c|c|l|l|}
\hline 1993 & 820 & $\begin{array}{l}\text { List of marine and estuarine fishes of mainland } \\
\text { Portugal, Azores and Madeira. }\end{array}$ & Magalhães \& Rogado (1993) \\
\hline 1997 & 510 & $\begin{array}{l}\text { List of marine fishes from the coastal waters } \\
\text { (Azores). }\end{array}$ & Santos et al. (1997) \\
\hline 2007 & 18 & $\begin{array}{l}\text { M. E. Costa published a list of Chondrichthyes } \\
\text { fish of South Portugal. }\end{array}$ & Costa (2007) \\
\cline { 2 - 4 } & 101 & $\begin{array}{l}\text { J. Gomes and S. Olim published a list of } \\
\text { Actinopterygii fish of South Portugal. }\end{array}$ & Gomes \& Olim (2007) \\
\hline 2008 & 226 & $\begin{array}{l}\text { List of marine fishes from the coastal waters } \\
\text { (Madeira). }\end{array}$ & Wirtz et al. (2008) \\
\hline 2010 & 543 & $\begin{array}{l}\text { Marine Fish (Chondrichthyes, Actinopterygii) } \\
\text { (Azores). }\end{array}$ & Porteiro et al. (2010) \\
\hline 2012 & 77 & $\begin{array}{l}\text { Annotated list of demersal fishes which occur in a } \\
\text { part of the area of the Azores EEZ. This list adds } \\
\text { 3 species to previous checklists of the Azores. }\end{array}$ & Menezes et al. (2012) \\
\hline
\end{tabular}

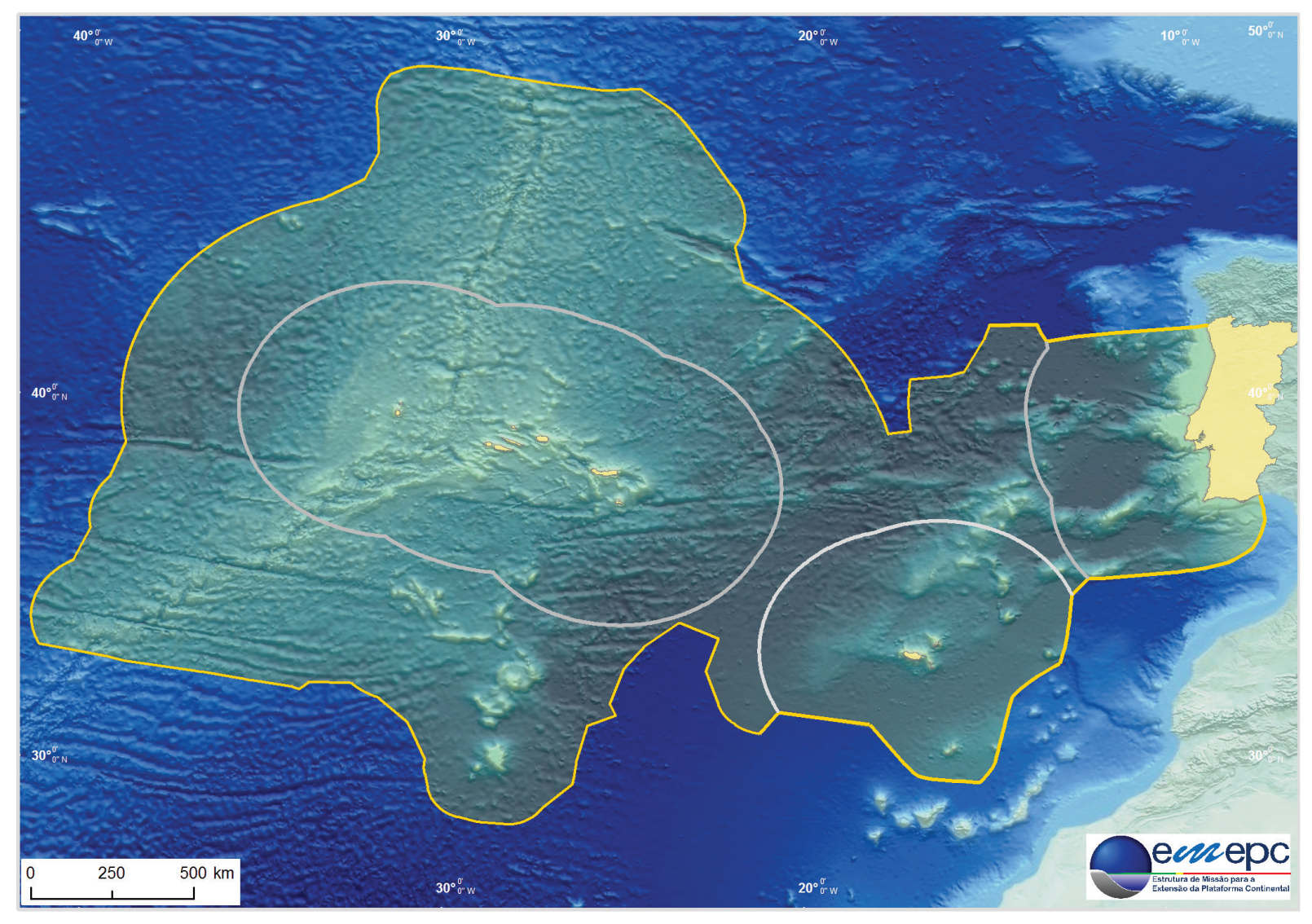

Fig. 1. Map of the study area, the Portuguese EEZ, that includes the territorial waters and the area proposed for the extension of the Portuguese continental shelf (source: EMEPC-Mission Structure for the Extension of the Continental Shelf). 
Table 2. List of electronic databases and collections databases consulted in this study.

\begin{tabular}{|c|c|c|c|}
\hline Acronym & Name & Author and/or access link & Accessed since \\
\hline AVG & $\begin{array}{l}\text { Aquário Vasco da Gama. Oceanographic } \\
\text { Collection of D. Carlos I, Lisboa, } \\
\text { Portugal }\end{array}$ & http://aquariovgama.marinha.pt & 2 March 2011 \\
\hline BMNH & Natural History Museum, London, U.K. & http://www.nhm.ac.uk & 2 March 2011 \\
\hline BOLDSYSTEMS & BOLD: The Barcode of Life Data System & $\begin{array}{l}\text { Ratnasingham \& Hebert (2007), } \\
\text { http://www.barcodinglife.org }\end{array}$ & 5 February 2012 \\
\hline CAS & $\begin{array}{l}\text { The Catalog of Fishes. California } \\
\text { Academy of Sciences San Francisco, } \\
\text { California, U.S.A. }\end{array}$ & $\begin{array}{l}\text { Eschmeyer (2013), } \\
\text { http://researcharchive.calacademy.org, } \\
\text { http://collections.calacademy.org }\end{array}$ & 2 March 2011 \\
\hline CLOFETA & $\begin{array}{l}\text { Checklist of the Fishes of the Eastern } \\
\text { Tropical Atlantic }\end{array}$ & Quéro et al. (1990) & 20 February 2012 \\
\hline CLOFNAM & $\begin{array}{l}\text { Check-list of the fish of the northeastern } \\
\text { Atlantic and of the Mediterranean }\end{array}$ & Hureau \& Monod (1979) & \\
\hline EMODnet & $\begin{array}{l}\text { European Marine Observation and Data } \\
\text { Network. Vlaams Instituut voor de Zee }\end{array}$ & $\begin{array}{l}\text { Hernandez (2013), } \\
\text { http://bio.emodnet.eu }\end{array}$ & 14 February 2013 \\
\hline FISHBASE & Global Information System on Fishes & $\begin{array}{l}\text { Froese \& Pauly (2012), } \\
\text { http://www.fishbase.org }\end{array}$ & 5 February 2012 \\
\hline FNAM & $\begin{array}{l}\text { Fishes of the northeastern Atlantic and } \\
\text { Mediterranean }\end{array}$ & Whitehead et al. $(1984,1986)$ & \\
\hline GBIF & Global Biodiversity Information Facility & http://data.gbif.org & 2 March 2011 \\
\hline GNI & Global Names Index & $\begin{array}{l}\text { Patterson et al. (2010), } \\
\text { http://gni.globalnames.org }\end{array}$ & 13 February 2013 \\
\hline ITIS & $\begin{array}{l}\text { Integrated Taxonomic Information } \\
\text { System }\end{array}$ & http://www.itis.gov & 2 March 2011 \\
\hline MB & $\begin{array}{l}\text { Museu Bocage, Zoologia e Antropologia } \\
\text { in Museu Nacional de História Natural } \\
\text { Universidade de Lisboa, Lisboa, Portugal }\end{array}$ & http://www.mnhn.ul.pt & 2 March 2011 \\
\hline $\mathrm{MCM}$ & $\begin{array}{l}\text { Museu Carlos Machado, Ponta Delgada, } \\
\text { Azores, Portugal }\end{array}$ & http://museucarlosmachado.azores.gov.pt & 2 March 2011 \\
\hline $\mathrm{MCZ}$ & $\begin{array}{l}\text { Museum of Comparative Zoology, } \\
\text { Harvard University, Ichthyology } \\
\text { Department, Cambridge, Massachusetts, } \\
\text { U.S.A. }\end{array}$ & http://www.mcz.harvard.edu & 2 March 2011 \\
\hline MHNF & $\begin{array}{l}\text { Museu de História Natural do Funchal, } \\
\text { Câmara; Municipal do Funchal, Funchal, } \\
\text { Madeira, Portugal }\end{array}$ & http://www1.cm-funchal.pt & 2 March 2011 \\
\hline MNHN & $\begin{array}{l}\text { Muséum National d'Histoire Naturelle, } \\
\text { Systématique et Évolution, Laboratoire } \\
\text { d'Ichthyologie Générale et Appliquée, } \\
\text { Paris, France }\end{array}$ & http://coldb.mnhn.fr & 2 March 2011 \\
\hline MOM & $\begin{array}{l}\text { Musée Oceanographique du Mónaco, } \\
\text { Mónaco }\end{array}$ & http://www.oceano.mc & 2 March 2011 \\
\hline NARMS & $\begin{array}{l}\text { North Atlantic Register for Marine } \\
\text { Species }\end{array}$ & $\begin{array}{l}\text { Vanden Berghe et al. (2005), } \\
\underline{\text { http://www.vliz.be }}\end{array}$ & 8 January 2012 \\
\hline NMNH & $\begin{array}{l}\text { National Museum of Natural History, } \\
\text { Division of Fishes Collections, } \\
\text { Washington D.C. }\end{array}$ & $\begin{array}{l}\text { Williams (2013), } \\
\text { http://collections.mnh.si.edu }\end{array}$ & 2 March 2011 \\
\hline OBIS & $\begin{array}{l}\text { Ocean Biogeographic Information } \\
\text { System }\end{array}$ & http://iobis.org & 2 March 2011 \\
\hline ROM & $\begin{array}{l}\text { Royal Ontario Museum, Department } \\
\text { of Natural History, Toronto, Ontario, } \\
\text { Canada }\end{array}$ & http://www.rom.on.ca & 2 March 2011 \\
\hline WoRMS & World Register of Marine Species & $\begin{array}{l}\text { WoRMS Editorial Board (2013), } \\
\text { http://www.marinespecies.org }\end{array}$ & 2 March 2011 \\
\hline
\end{tabular}




\section{Material and methods}

In drafting of the present checklist, we included historical records reporting on the occurrence of marine fish species from Portugal and published between the $18^{\text {th }}$ and the early $21^{\text {st }}$ centuries (Table 1 ). In addition to the publications listed in Table 1, other sources were consulted, such as electronic databases and records from museum collections where the specimens captured in the study area are deposited (Table 2).

The geographic areas considered here are: the territorial waters, the Portuguese Economic Exclusive Zone-EEZ, and the proposed area for the extension of the Portuguese continental shelf (PECS) (Fig. 1). The species with occurences in the proposed area for the extension are indicated as "in the PECS area".

We followed Nelson's (2006) classification system for taxonomic categories ${ }^{1}$, Froese \& Pauly (2012) for the scientific nomenclature ${ }^{2}$, and we used FAO names based on the Aquatic Science and Fisheries Information System (ASFIS) for the majority of the English common names. The taxonomic categories considered in the checklist were: superclass, class, order, family and species. Within each family, species were sorted alphabetically.

For each species, we provide the English common name according to FAO's global designation, or the English vernacular in case no name was provided by FAO. "No common name" indicates that the common name is not available. Occurrences within a geographic area were marked as: mainland Portugal (1), the Archipelago of the Azores (2), and the Madeira Archipelago (3). The use of one or more of these symbols, (1) (2) (3), indicates the presence of the same species in each of the mentioned geographic areas. Local names, when available in registers and documents, are reported followed by symbols for the species' relative geographic area of occurrence. Taking into account the extension of the study area, the number of fishing communities covered, and the diversity and richness of local fish names used in a certain area, we decided to indicate, for each species, the most frequently used vernacular names in Portuguese. Thus, for a given geographic area and species more than one common name can be provided.

The most important references for Portuguese vernacular fish names are Albuquerque (1954-1956), Castro (1967), Sanches $(1986,1989)$ and the list of common names of the Portuguese legislation (Annex I and II of the "Portaria $n^{\circ} 587 / 2006$ " - List of Authorized Commercial Names for Fishery and Aquaculture Products, and "Declaração de Rectificação n ${ }^{\circ}$ 52/2006").

The species present in the collection of the Museu de História Natural - Museu Bocage ${ }^{3}$, University of Lisbon, in the collection of the Science Museum of the University of Coimbra, and in "Oceanographic Collection of D. Carlos I" - Aquário Vasco da Gama, are marked with an asterisk (*). The species archived in the collection of the Department of Oceanography and Fisheries of the University of the Azores (Horta) and in the Museu Carlos Machado (São Miguel) are marked with a diamond $(\diamond)$. The species stored in the collection of the Museu de História Natural do Funchal are marked with a square $(\square)$.

As criteria for inclusion, we considered all species recorded in the EEZ plus the Portuguese territorial waters, and the proposed extension area of the Portuguese continental shelf. All records of occurrence from the PECS are indicated. Furthermore, all species considered native to one of the three EEZ's (mainland Portugal, Azores and Madeira), but without records, are indicated.

\footnotetext{
${ }^{1}$ With the exception of the families Arhynchobatidae (Aschliman et al. 2012; Naylor et al. 2012), Howellidae (Prokofiev 2006), Phycidae and Gadidae (Nolf 2013).

${ }^{2}$ In addition, other sources (electronic databases) were also consulted, such as: ITIS (Integrated Taxonomic Information System); CAS (California Academy of Sciences, Eschmeyer 2013); GNI (Global Names Index; Patterson et al. 2010).

${ }^{3}$ The Museu Bocage (MB) was completely destroyed by fire in March 1978 and consequently all the types of fishes preserved there to that date were lost.
} 
For the biogeographical distribution we referred to Ellis et al. (2008). In terms of horizontal habitat preference, fish were broadly classified as: coastal, shelf, inner and outer shelf, slope, oceanic and oceanic/shelf (adapted from Ellis et al. 2008). Considering the fish distribution in the vertical habitat we used the following terminology: benthic, benthopelagic, mesopelagic, pelagic, epipelagic, demersal, bathydemersal, and reef-associated (adapted from Ellis et al. 2008).

In the list, NM stands for nautical miles.

\section{Results}

\section{Global appraisal of species richness, biogeographic groups, and habitat distribution}

The checklist comprises a total of 1191 species, representing 3 superclasses, 4 classes, 42 orders, 212 families and 617 genera (Table 3). The order Perciformes shows the highest diversity, with 54 families, 162 genera and 299 species. Of all the families, Stomiidae ( 80 species), Myctophidae (71 species) and Macrouridae (37 species) were the richest (Table 3).

The highest number of species was detected off the Azores (857), followed by Madeira (766), and the Portuguese mainland (734). A total of 133 species occurrences were included for the PECS area (2 in the Portuguese mainland, 117 off the Azores and 14 in Madeira), two of which are common to the Azores and Madeira extensions. For the first time we report three species observed off mainland Portugal (Bajacalifornia megalops (Lütken, 1898), Fistularia petimba Lacepède, 1803 and Scombrolabrax heterolepis Roule, 1921) and, for the whole study area, thirty records are annotated as doubtful.

Table 3. Number of valid families, genera and species of fish from Portugal included in the checklist, according to Nelson's (2006) classification.

\begin{tabular}{|l|c|c|c|l|c|c|c|}
\hline Order & Families & Genera & Species & Order & Families & Genera & Species \\
\hline Myxiniformes & 1 & 1 & 1 & Stomiiformes & 5 & 38 & 118 \\
Petromyzontiformes & 1 & 2 & 2 & Ateleopodiformes & 1 & 1 & 1 \\
Chimaeriformes & 2 & 3 & 6 & Aulopiformes & 10 & 24 & 49 \\
Orectolobiformes & 1 & 1 & 1 & Myctophiformes & 2 & 24 & 74 \\
Lamniformes & 5 & 7 & 10 & Lampriformes & 6 & 7 & 8 \\
Carcharhiniformes & 5 & 11 & 28 & Polymixiiformes & 1 & 1 & 1 \\
Hexanchiformes & 2 & 3 & 4 & Gadiformes & 6 & 40 & 73 \\
Echinorhiniformes & 1 & 1 & 1 & Ophidiiformes & 5 & 26 & 35 \\
Squaliformes & 6 & 15 & 27 & Batrachoidiformes & 1 & 1 & 1 \\
Squatiniformes & 1 & 1 & 2 & Lophiiformes & 14 & 30 & 64 \\
Torpediniformes & 1 & 1 & 3 & Mugiliformes & 1 & 4 & 6 \\
Pristiformes & 1 & 1 & 1 & Atheriniformes & 1 & 1 & 3 \\
Rajiformes & 3 & 9 & 21 & Beloniformes & 4 & 8 & 16 \\
Myliobatiformes & 3 & 9 & 12 & Stephanoberyciformes & 6 & 16 & 31 \\
Elopiformes & 1 & 1 & 1 & Beryciformes & 5 & 7 & 10 \\
Albuliformes & 2 & 6 & 14 & Zeiformes & 5 & 7 & 8 \\
Anguilliformes & 10 & 33 & 54 & Gasterosteiformes & 5 & 9 & 20 \\
Saccopharyngiformes & 4 & 4 & 13 & Scorpaeniformes & 8 & 20 & 35 \\
Clupeiformes & 2 & 5 & 7 & Perciformes & 54 & 162 & 297 \\
Argentiniformes & 6 & 39 & 63 & Pleuronectiformes & 7 & 22 & 37 \\
Salmoniformes & 1 & 2 & 3 & Tetraodontiformes & 6 & 14 & 28 \\
\hline
\end{tabular}


As a result of the update of the two most comprehensive checklists of marine fishes from Portugal published in 1954 and 1993 (Albuquerque 1954-1956; Magalhães \& Rogado 1993), the Portuguese ichthyofauna included in the three EEZ's (mainland Portugal, Azores and Madeira) increased by 230 species $(27.8 \%)$ or 238 species (29.0\%), compared to Fishbase (2012) and to the last checklist of marine and estuarine fishes of Portugal (1993), respectively.

In terms of biogeographical affinities, 9 of the 13 groups referred to by Ellis et al. (2008) were identified, namely the Atlantic, the Lusitanian, the African, the Boreal, the Mediterranean, the Macaronesian, the Atlantic/African, the Mediterranean/African and the Arctic groups. Among these, the Atlantic, the Lusitanian and the African groups are the largest represented, with a total of $46.01 \%, 21.49 \%$ and $5.96 \%$ respectively, when EEZ and the proposed area of extension are considered. The remaining groups are residual (Table 4). Because 209 species currently lack biogeographical classification, they were included in the uncertain group.

Regarding the vertical habitat preferred by fish, all 9 groups referred by Ellis et al. (2008) were identified, namely the demersal, the mesopelagic, the bathydemersal, the bathypelagic, the benthopelagic, the reefassociated, the pelagic, the epipelagic, and the benthic group (Table 5). In what concerns the horizontal habitat, all 7 groups suggested by Ellis et al. (2008) were characterized. The oceanic is the most important group, followed by the shelf group, the slope group, the inner shelf group, the coastal group, the outer shelf group, and the oceanic/shelf group (Table 6). Because 11 species currently lack vertical habitat classification and 187 species lack horizontal habitat classification, they were included in the uncertain group and an unknown group respectively.

Table 4. Percentage of species by biogeographic group. Number of species is provided within parentheses for each area, including the EEZ and the area corresponding to the proposed extention of the continental shelf (PECS).

\begin{tabular}{|c|c|c|c|c|c|c|c|c|c|c|}
\hline Species Number & 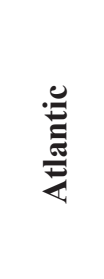 & 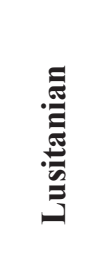 & 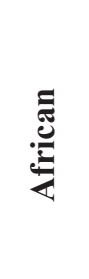 & $\begin{array}{l}\overline{\mathscr{J}} \\
\stackrel{0}{0}\end{array}$ & 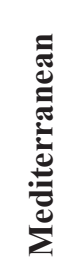 & 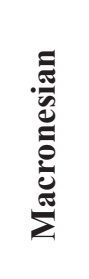 & 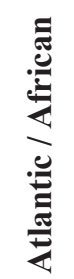 & 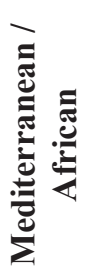 & 苞 & \\
\hline Total EEZ $(\mathrm{n}=1058)$ & 41.65 & 20.82 & 5.63 & 2.43 & 2.18 & 1.76 & 1.51 & 0.08 & 0.08 & 12.77 \\
\hline Total PECS $(\mathrm{n}=133)$ & 4.37 & 0.67 & 0.34 & 0.42 & 0.42 & - & 0.08 & - & - & 4.79 \\
\hline Mainland Portugal EEZ $(\mathrm{n}=732)$ & 45.37 & 33.24 & 5.45 & 4.22 & 2.72 & 0.54 & 0.82 & 0.14 & - & 7.23 \\
\hline Mainland Portugal PECS $(n=2)$ & 0.27 & - & - & - & - & - & - & - & - & - \\
\hline Azores EEZ $(\mathrm{n}=740)$ & 49.94 & 15.40 & 4.55 & 1.17 & 1.05 & 2.10 & 1.75 & - & 0.12 & 10.37 \\
\hline Azores PECS $(\mathrm{n}=117)$ & 5.37 & 0.82 & 0.35 & 0.47 & 0.47 & - & 0.12 & - & - & 5.95 \\
\hline Madeira EEZ $(n=752)$ & 53.39 & 20.37 & 6.79 & 0.91 & 1.17 & 2.22 & 1.70 & - & - & 11.62 \\
\hline Madeira PECS $(n=14)$ & 0.39 & 0.39 & 0.13 & 0.13 & 0.13 & - & - & - & - & 0.66 \\
\hline
\end{tabular}


Table 5. Proportion of fish species according to preferred vertical habitat, distributed by the total study area, EEZ plus territorial waters, and the area corresponding to the proposed extension of the Portuguese continental shelf (PECS).

\begin{tabular}{|c|c|c|c|c|c|c|c|c|c|c|}
\hline & 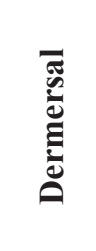 & 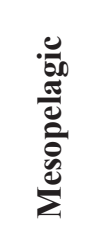 & 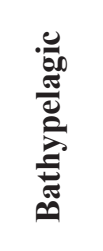 & 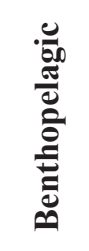 & 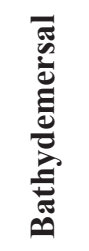 & 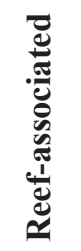 & 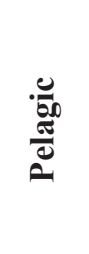 & 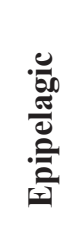 & 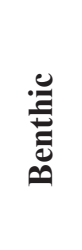 & \\
\hline Total $(\mathrm{n}=1191)$ & 25.61 & 19.14 & 13.77 & 12.34 & 9.66 & 7.39 & 6.21 & 4.87 & 0.08 & 0.93 \\
\hline $\operatorname{EEZ}(\mathrm{n}=1058)$ & 24.27 & 17.13 & 9.57 & 11.25 & 8.73 & 6.88 & 5.37 & 4.87 & 0.08 & 0.77 \\
\hline PECS $(n=133)$ & 1.34 & 2.02 & 4.20 & 1.09 & 0.92 & 0.50 & 0.84 & - & - & 0.17 \\
\hline
\end{tabular}

Table 6. Proportion of fish species by preferred horizontal habitat, distributed by the total study area, EEZ plus territorial waters, and the area corresponding to the proposed extension of the Portuguese continental shelf (PECS).

\begin{tabular}{|c|c|c|c|c|c|c|c|c|}
\hline & 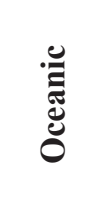 & 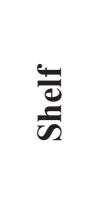 & $\frac{\ddot{a}}{\ddot{a}}$ & 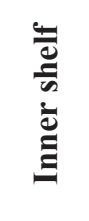 & $\begin{array}{l}\overline{5} \\
\text { Uूँ } \\
\tilde{E}\end{array}$ & $\begin{array}{l}\frac{\bar{\Xi}}{0} \\
\frac{0}{0} \\
\overline{0}\end{array}$ & 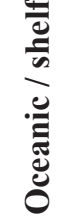 & 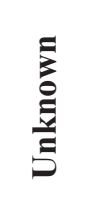 \\
\hline Total $(n=1191)$ & 37.45 & 16.71 & 13.77 & 7.47 & 5.88 & 2.43 & 0.59 & 15.70 \\
\hline $\operatorname{EEZ}(\mathrm{n}=1058)$ & 32.91 & 16.12 & 12.26 & 7.30 & 5.88 & 2.35 & 0.42 & 11.68 \\
\hline PECS (n =133) & 4.53 & 0.59 & 1.51 & 0.17 & - & 0.08 & 0.17 & 4.03 \\
\hline
\end{tabular}

\section{Annotated Checklist}

Superclass Myxinomorphi

Class Myxini

Order Myxiniformes

Family Myxinidae

* Myxine glutinosa Linnaeus, 1758 - Hagfish; Enguia-de-casulo (1)

Superclass Petromyzontomorphi

Class Petromyzontida

Order Petromyzontiformes

Family Petromyzontidae

Lampetra fluviatilis (Linnaeus, 1758) - River lamprey; Lampreia-do-rio (1)

* Petromyzon marinus Linnaeus, 1758 - Sea lamprey; Lampreia-do-mar(1) (3) 
Superclass Gnathostomata

Class Chondrichthyes

Sub-Class Holocephali

Order Chimaeriformes

Family Rhinochimaeridae

Rhinochimaera atlantica Holt \& Byrne, 1909 - Straightnose rabbitfish; (1) (2)

The species $R$. atlantica is regarded as being present off mainland Portugal, based on its geographical distribution (Ebert \& Stehmann 2013).

\section{Family Chimaeridae}

* $\square$ Chimaera monstrosa Linnaeus, 1758 - Rabbit-fish; Ratazana (1), Quimera (2) (3)

$\square \quad$ Hydrolagus affinis (de Brito Capello, 1868) - Smalleyed rabbitfish; Ratazana-da-fundura (1), (2) (3) Hydrolagus lusitanicus Moura, Figueiredo, Bordalo-Machado, Almeida \& Gordo, 2005 - No common name; (1)

Hydrolagus mirabilis (Collett, 1904) - Large-eyed rabbitfish; (1) (2)

The species H. mirabilis is regarded as being present off mainland Portugal, based on its geographical distribution (Ebert \& Stehmann 2013).

Hydrolagus pallidus Hardy \& Stehmann, 1990 - Ghost shark; (2)

\section{Sub-Class Elasmobranchii \\ Order Orectolobiformes \\ Family Rhincodontidae}

Rhincodon typus Smith, 1829 - Whale shark; Tubarão-baleia (1) (2), Pintado(2), (3)

Order Lamniformes

Family Odontaspididae

Odontaspis ferox (Risso, 1810) - Smalltooth sand tiger; Tubarão-areia, (2) (3)

$\square \quad$ Odontaspis noronhai (Maul, 1955) - Bigeye sand tiger shark; (3)

Family Mitsukurinidae

* Mitsukurina owstoni Jordan, 1898 - Goblin shark; Tubarão-demónio(1), (3)

Family Alopiidae

* Alopias superciliosus Lowe, 1841 - Bigeye thresher; Tubarão-raposo-olhudo(1), Tubarão-raposo (2), (3)

* $\square$ Alopias vulpinus (Bonnaterre, 1788) - Thresher; Tubarão-raposo (1) (2), Romano or Romão (2), Peixerato (3)

\section{Family Cetorhinidae}

* $\quad$ Cetorhinus maximus (Gunnerus, 1765) - Basking shark; Tubarão-frade (1) (2), Peixe-frade(2) (3)

Family Lamnidae

* Carcharodon carcharias (Linnaeus, 1758) - Great white shark; Tubarão-de-São-Tomé(1), Tubarãobranco (2), (3) 
* Isurus oxyrinchus Rafinesque, 1810 - Shortfin mako; Tubarão-anequim(1), Rinquim(2), Marracho(2) (3), Anequim (3)

Isurus paucus Guitart, 1966 - Longfin mako; Tubarão-anequim-de-gadanha (1), Marrajo-negro(2)

* Lamna nasus (Bonnaterre, 1788) - Porbeagle; Tubarão-sardo (1), Marracho(2) (3)

The species L. nasus is indicated as native to Madeira (Compagno 1984a), although Wirtz et al. (2008) considered the presence of this species off Madeira as doubtful.

\section{Order Carcharhiniformes \\ Family Scyliorhinidae}

$\square \quad$ Apristurus laurussonii (Saemundsson, 1922) - Iceland catshark; (2) (3)

Apristurus manis (Springer, 1979) - Ghost catshark; Tubarão-gato-fantasma(2)

Apristurus profundorum (Goode \& Bean, 1896) - Deep-water catshark; (2)

* Galeus atlanticus (Vaillant, 1888) - Atlantic sawtail cat shark; (1)

* Galeus melastomus Rafinesque, 1810 - Blackmouth catshark; Leitão(1) (3), (2)

The presence of this species off the Azores needs further evaluation (Santos et al. 1997). According to Ebert \& Stehmann (2013), the occurrence of G. melastomus off the Azores is possible.

Galeus murinus (Collett, 1904) - Mouse catshark; (1) (2)

The species G. murinus is regarded as being present off mainland Portugal, based on its geographical distribution (Ebert \& Stehmann 2013).

* $\diamond$ Scyliorhinus canicula (Linnaeus, 1758) - Small-spotted catshark; Pata-roxa(1), (2) (3)

* Scyliorhinus stellaris (Linnaeus, 1758) - Nursehound; Pata-roxa-gata (1), (2)

Family Pseudotriakidae

* Pseudotriakis microdon de Brito Capello, 1868 - False catshark; Tubarão-mona(1), Mamôna(2), Mona (3)

\section{Family Triakidae}

* Galeorhinus galeus (Linnaeus, 1758) - Tope shark; Cação (1) (2) (3), Perna-de-moça (1)

Mustelus asterias Cloquet, 1821 - Starry smooth-hound; Cação-pintado (1), (3)

* $\square$ Mustelus mustelus (Linnaeus, 1758) - Smooth-hound; Cação-liso(1), (2), Caneja (3)

The presence of this species off the Azores needs further documentation (Santos et al. 1997). According to Ebert \& Stehmann (2013), the occurrence of M. mustelus off the Azores is possible.

Mustelus punctulatus Risso, 1827 - Blackspotted smooth-hound; (1)

Family Carcharhinidae

Carcharhinus brachyurus (Günther, 1870) - Copper shark; Tubarão-cobre (1)

Carcharhinus brevipinna (Müller \& Henle, 1839) - Spinner shark; Tubarão-tecelão (1), (2)

Arruda (1997) considered the occurrence of this species off the Azores as doubtful. Quéro et al. (2003) indicated the presence of the species off Portugal, without referring to the geographical area of occurrence. According to Ebert \& Stehmann (2013), the presence of C. brevipinna off southern Portugal is possible.

Carcharhinus falciformis (Müller \& Henle, 1839) - Silky shark; Tubarão-luzidio (1), (2) (3)

The occurrence of this species off Madeira needs confirmation (Wirtz et al. 2008). Ebert \& Stehmann (2013) indicated its presence off Madeira, based on known geographical distribution. Arruda (1997) considered the presence of C. falciformis off the Azores doubtful.

$\square \quad$ Carcharhinus galapagensis (Snodgrass \& Heller, 1905) - Galapagos shark; Tubarão-dos-Galápos(2), (3) According to Ebert \& Stehmann (2013), the species Carcharhinus obscurus is very closely related to this species (C. galapagensis) and they are difficult to distinguish from each other. Recent molecular studies suggest that these two species may in fact be a single one, with one of the two forms being found far from 
landmasses (C. galapagensis) and the other one (C. obscurus) occurring in association with continental shelves and upper slopes (G. Naylor, pers comm. in Ebert \& Stehmann 2013). Studies are currently ongoing to determine the relationship between these two species.

Carcharhinus leucas (Müller \& Henle, 1839) - Bull shark; Tubarão-buldogue, (2)

$\square \quad$ Carcharhinus limbatus (Müller \& Henle, 1836) - Blacktip shark; Tubarão-de-pontas-negras (1), (2), Tubarão or Anequim (3)

Arruda (1997) considered a doubtful presence of C. limbatus off the Azores. Quéro et al. (2003) indicated the presence of the species in Portugal, without mentioning the geographical area of occurrence. Ebert \& Stehmann (2013) indicated the presence of the species to southern mainland Portugal, based on its geographical distribution.

$\square \quad$ Carcharhinus longimanus (Poey, 1861) - Oceanic whitetip shark; Tubarão-de-pontas-brancas (1) (2), Marracho (2), (3)

- Carcharhinus obscurus (Lesueur, 1818) - Dusky shark; Tubarão-faqueta(1), (2), Faqueta (3)

According to Ebert \& Stehmann (2013) the records of this species from the Azores might concern $C$. galapagensis. Details are given above for the species $C$. galapagensis.

Carcharhinus plumbeus (Nardo, 1827) - Sandbar shark; Tubarão-corre-costa (1), (2) (3)

Arruda (1997) considered a doubtful presence of this species off the Azores. According to Ebert \& Stehmann (2013) the occurrence of C. plumbeus in Madeira is possible.

$\diamond \quad$ Galeocerdo cuvier (Péron \& Lesuer, 1822) - Tiger shark; Tubarão-tigre(2), (3)

Ebert \& Stehmann (2013) indicated the presence of the species in Madeira based on its geographical distribution.

* $\square$ Prionace glauca (Linnaeus, 1758) - Blue shark; Tintureira (1) (2) (3), Tubarão-azul(2)

Rhizoprionodon acutus (Rüppell, 1837) - Milk shark; Tubarão-bicudo(3)

The species R. acutus is indicated as native to Madeira (Compagno 1984b), but its presence was not confirmed by any geographic records. Wirtz et al. (2008) considered it very doubtful for Madeira.

\section{Family Sphyrnidae}

Sphyrna lewini (Griffith \& Smith, 1834) - Scalloped hammerhead; Tubarão-martelo-recortado (1) (2) (3) Sphyrna mokarran (Rüppell, 1837) - Great hammerhead; Tubarão-martelo-gigante (1)

The species $S$. mokarran is indicated as present off mainland Portugal, based on its geographical distribution (Ebert \& Stehmann 2013).

* $\square$ Sphyrna zygaena (Linnaeus, 1758) - Smooth hammerhead; Tubarão-martelo (1) (2), Cornuda or Peixe-martelo(2), Cornuda (3)

Order Hexanchiformes

Family Chlamydoselachidae

* Chlamydoselachus anguineus Garman, 1884 - Frilled shark; Tubarão-cobra (1), (2) (3)

Family Hexanchidae

* Heptranchias perlo (Bonnaterre, 1788)-Sharpnose sevengill shark; Boca-doce (1), Bico-doce (1) (2) (3), Albafar-bravo(2)

Hexanchus griseus (Bonnaterre, 1788) - Bluntnose sixgill shark; Tubarão-albafar(1), Albafar(1) (2) (3), Tubarão-albafar-bravo (2)

Hexanchus nakamurai Teng, 1962 - Bigeyed sixgill shark; (1)

This species is regarded as being present off mainland Portugal based on its geographical distribution (Ebert \& Stehmann 2013). 
Order Echinorhiniformes

Family Echinorhinidae

* Echinorhinus brucus (Bonnaterre, 1788) - Bramble shark; Tubarão-prego (1), Peixe-prego (2)

Order Squaliformes

Family Squalidae

According to Ebert \& Stehmann (2013), three species of the genus Squalus are recognized in the North Atlantic (S. acanthias, S. blainvillei and S. megalops), but S. blainvillei and S. megalops may represent a species complex. The taxonomic arrangement of Squalus species is provisional, pending the review and resolution of the $S$. blainvillei and $S$. megalops complexes.

* Squalus acanthias Linnaeus, 1758 - Piked dogfish; Galhudo-malhado(1), (2) (3)

The presence of this species off the Azores needs further confirmation (Santos et al. 1997). According to Ebert \& Stehmann (2013) its occurrence off the Azores is probable.

* $\square$ Squalus blainvillei (Risso, 1827) - Longnose spurdog; Galhudo (1), (2)

Arruda (1997) considered the presence of this species off the Azores as doubtful.

Squalus megalops (Macleay, 1881) - Shortnose spurdog; Galhudo-de-focinho-curto (1)

The species S. megalops is regarded as being present to the south of mainland Portugal, based on its geographical distribution (Ebert \& Stehmann 2013).

Family Centrophoridae

Muñoz-Chapuli \& Ramo (1989) reviewed the systematics of Centrophorus from the eastern North Atlantic and recognized four different species: C. squamosus, C. granulosus, C. lusitanicus and $C$. niaukang. However, recent studies indicated that $C$. niaukang may be restricted to the western IndoPacific (Ebert \& Stehmann 2013). The taxonomic arrangement of Centrophorus species is provisional, and a comprehensive systematic review of Centrophorus, including detailed descriptions of external morphological, anatomical and molecular characters (Muñoz-Chapuli \& Ramo 1989), are necessary both to elucidate the variation and interrelationships of Centrophorus species and to rectify several outstanding problems within the genus. According to Ebert \& Stehmann (2013), the species Centrophorus uyato Rafinesque, 1810, is not actually a Centrophorus species, but rather a Squalus of uncertain identity.

* Centrophorus granulosus (Bloch \& Schneider, 1801) - Gulper shark; Barroso(1)(2), Quelma(2), Ramudo (3)

* Centrophorus lusitanicus Bocage \& Capello, 1864 - Lowfin gulper shark; (1), Ramudo(3)

$\square \quad$ Centrophorus niaukang Teng, 1959 - Taiwan gulper shark; (3)

* Centrophorus squamosus (Bonnaterre, 1788) - Leafscale gulper shark; Lixa (1), Lixa-de-escama (2), Xara-branca (3)

* Deania calcea (Lowe, 1839) - Birdbeak dogfish; Sapata(1) (2) (3), Pífaro, Pife or Tutia(2)

Deania hystricosa (Garman, 1906) - Rough longnose dogfish; (1) (2) (3)

* Deania profundorum (Smith \& Ratcliffe, 1912) - Arrowhead dogfish; (1), Sapata or Sapa-branca(2), (3)

Family Etmopteridae

Centroscyllium fabricii (Reinhardt, 1825) - Black dogfish; (1) (2)

$\square \quad$ Etmopterus princeps Collet, 1904 - Great lanternshark; (1), Lixinha-da-fundura-grada (2), (3)

* Etmopterus pusillus (Lowe, 1839) - Smooth lanternshark; Xarinha-preta (1), Lixinha-da-fundura or Quelmazinha (2), Gata-preta or Xara-preta (3) 
* Etmopterus spinax (Linnaeus, 1758) - Velvet belly; Lixinha-da-fundura (1) (2) (3), Quelmazinha (2), Lixinha (3)

\section{Family Somniosidae}

* Centroscymnus coelolepis Barbosa du Bocage \& de Brito Capello, 1864 - Portuguese dogfish; Carocho (1) (2), Tubarão-português (2), Xara-preta (3)

$\square \quad$ Centroscymnus owstonii Garman, 1906 - Roughskin dogfish; (1) (3), Xara-preta-de-natura(2) The species Centroscymnus cryptacanthus Regan, 1906 is a synonym of C. owstonii (Compagno 2003).

* Centroselachus crepidater (Bocage \& Capello, 1864) - Longnose velvet dogfish; Sapata-preta (1) (2), Sapata-de-natura (3) Scymnodalatias garricki Kukuev \& Konovalenko, 1988 - Azores dogfish; (2)

* Scymnodon ringens Barbosa du Bocage \& de Brito Capello, 1864 - Knifetooth dogfish; Arreganhada (1), Boca (3)

$\square$ Somniosus microcephalus (Bloch \& Schneider, 1801) - Greenland shark; Tubarão-daGronelândia (1) (2), (3)

Somniosus rostratus (Risso, 1827) - Little sleeper shark; Pailona (1), (2) (3)

$\square \quad$ Zameus squamulosus (Günther, 1877) - Velvet dogfish; Arreganhada-de-focinho-comprido(1) (2), Arreganhada(2), (3)

The species Scymnodon obscurus (Vaillant, 1888) is a synonym of Z. squamulosus (Compagno 2003).

\section{Family Oxynotidae}

* Oxynotus centrina (Linnaeus, 1758) - Angular roughshark; Peixe-porco (1)

Oxynotus paradoxus Frade, 1929 - Sailfin roughshark; Peixe-porco-de-vela (1), (2) (3)

The species $O$. paradoxus is regarded as being present in Madeira, based on its geographical distribution (Ebert \& Stehmann 2013).

\section{Family Dalatiidae}

* $\square$ Dalatias licha (Bonnaterre, 1788) - Kitefin shark; Gata (1) (3), Gata-lixa or Gato (2), Trabolha (3) Isistius plutodus Garrick \& Springer, 1964 - Largetooth cookiecutter shark; (2) This species is reported to occur off the Azores, in the PECS area (95 NM southwest of the Olympus Knoll), SAMC, SAMS-SHARKS-006797, $28.5333^{\circ} \mathrm{W}, 43.9667^{\circ} \mathrm{N}$.

$\square \quad$ Squaliolus laticaudus Smith \& Radcliffe, 1912 - Spined pygmy shark; Tubarão-anão(2), (3)

Order Squatiniformes

Family Squatinidae

Squatina oculata Bonaparte, 1840 - Smoothback angelshark; Anjo-de-malhas (1)

* Squatina squatina (Linnaeus, 1758) - Angelshark; Anjo (1)

Order Torpediniformes

Family Torpedinidae

* $\square$ Torpedo (Torpedo) marmorata Risso, 1810 - Spotted torpedo; Tremelga-marmoreada (1), Tormentim or Tremedeira (3)

* Torpedo (Tetronarce) nobiliana Bonaparte, 1835 - Electric ray; Tremelga-negra (1), Arraia or Tremelga (2), Tormentim, Dormideira or Tremedeira (3)

* $\square$ Torpedo (Torpedo) torpedo (Linnaeus, 1758) - Common torpedo; Tremelga-de-olhos (1), (2), Tormentim, Dormideira or Tremedeira (3) 
The presence of this species off the Azores needs further documentation (Santos et al. 1997).

Order Pristiformes

Family Pristidae

Pristis pristis (Linnaeus, 1758) - Common sawfish; Espadarte-serra (1), Espadarte(3)

Order Rajiformes

Family Rhinobatidae

Rhinobatos (Glaucostegus) cemiculus Geoffroy Saint-Hilaire, 1817 - Blackchin guitarfish; Violabarba-negra (1)

* Rhinobatos (Rhinobatos) rhinobatos (Linnaeus, 1758) - Common guitarfish; Viola (1)

Family Arhynchobatidae

Bathyraja pallida (Forster, 1967) - Pale ray; (2)

Bathyraja richardsoni (Garrick, 1961) - Richardson's ray; (2)

Family Rajidae

Amblyraja radiata (Donovan, 1808) - Starry ray; Raia-repregada (1) (2)

The species $A$. radiata is reported to occur off the Azores, in the PECS area (70 NM southwest of Albany

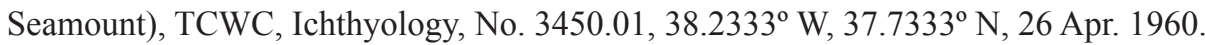

* Dipturus batis (Linnaeus, 1758) - Blue skate; Raia-oirega, Airoga, Arrai or Raia

Based on morphological analyses and on molecular phylogenetic relationships, Iglésias et al. (2010) revealed the existence of four distinct species in the northeastern Atlantic (namely D. cf. flossada, D. cf. intermedia, D. oxyrinchus and D. nidarosiensis). The records of D. batis off mainland Portugal, off the Azores and off Madeira can only be reclassified upon the formal revision of the Dipturus species from the northeastern Atlantic, and taking into consideration the resurrections and the fully documented re-descriptions of both Dipturus sp. cf. flossada (Risso, 1826) and of Dipturus sp. cf. intermedia (Parnell, 1837). Therefore, the species $D$. batis was not counted in the total number of species in the current list.

$* \diamond \square$ Dipturus oxyrinchus (Linnaeus, 1758) - Longnosed skate; Raia-bicuda (1), Arrai(2), Raia (2) (3)

* Leucoraja circularis (Couch, 1838) - Sandy ray; Raia-de-São-Pedro(1) Leucoraja fullonica (Linnaeus, 1758) - Shagreen ray; Raia-pregada (1) (2), Arraia or Raia (2), Raia (3)

* Leucoraja naevus (Müller \& Henle, 1841) - Cuckoo ray; Raia-de-dois-olhos (1) Neoraja iberica Stehmann, Séret, Costa \& Baro, 2008 - Iberian pygmy skate; Raia-pigméiaibérica (1)

* Raja asterias Delaroche, 1809 - Starry ray; Raia-pintada(1)

* Raja brachyura Lafont, 1873 - Blonde ray; Raia-pontuada (1) (2), Arraia or Raia (2), Raia (3)

* $\square$ Raja clavata Linnaeus, 1758 - Thornback ray; Raia-lenga (1) (2), (3)

$\square \quad$ Raja maderensis Lowe, 1839 - Madeira ray; Raia-da-Madeira(1), Arraia(2), Raia(2) (3)

* Raja microocellata Montagu, 1818 - Small-eyed ray; Raia-zimbreira(1), Arrai or Raia(2) The presence of this species off the Azores needs further documentation (Santos et al. 1997).

* Raja miraletus Linnaeus, 1758 - Brown ray; Raia-de-quatro-olhos (1), (3)

* $\square$ Raja montagui Fowler, 1910 - Spotted ray; Raia-manchada (1)

* Raja undulata Lacepède, 1802 - Undulate ray; Raia-curva (1) Rajella bathyphila (Holt \& Byrne, 1908) - Deep-water-ray; (2) Rajella bigelowi (Stehmann, 1978) - Bigelow's ray; (1), Arraia or Raia(2) The species $R$. bigelowi is regarded as being present off mainland Portugal, based on its geographical distribution (Ebert \& Stehmann 2013).

* $\square$ Rostroraja alba (Lacepède, 1803) - Bottlenosed skate; Raia-tairoga (1), (3) 


\section{Order Myliobatiformes \\ Family Dasyatidae}

$\diamond$ Dasyatis centroura (Mitchill, 1815) - Roughtail stingray; Uge-de-cardas(1), Ratão(2), (3)

* Dasyatis pastinaca (Linnaeus, 1758) - Common stingray; Uge(1) (2), Rato-do-mar(2), Ratão (2) (3)

$\square \quad$ Pteroplatytrygon violacea (Bonaparte, 1832) - Pelagic stingray; Uge-violeta (1), Ratão or Raiapelágica(2), (3)

$\diamond \square$ Taeniura grabata (Geoffroy Saint-Hilaire, 1817) - Roundstingray; Ratão(2) (3)

Family Gymnuridae

$\square \quad$ Gymnura altavela (Linnaeus, 1758) - Spiny butterfly ray; Uge-manta (1), Raia or Andorinha-do$\operatorname{mar}(3)$

Gymnura hirundo (Lowe, 1843) - Madeira butterfly ray; Raia-borboleta (3)

Compagno (1999) considered the validity of this species as doubtful.

Family Myliobatidae

$\square \quad$ Manta birostris (Walbaum, 1792) - Giant manta; Manta (1) (3), Urjamanta or Marona(2), Urjamanta (3)

- Mobula mobular (Bonnaterre, 1788) - Devil fish; Jamanta (1) (2) (3), Jimanta, Uge or Uja(2), Urjamanta (3)

Mobula tarapacana (Philippi, 1892) - Chilean devil ray; Manta-cornuda (2), (3)

* Myliobatis aquila (Linnaeus, 1758) - Common eagle ray; Ratão-águia (1), Arreião or Ratão(2), Raia(3)

Pteromylaeus bovinus (Geoffroy Saint-Hilaire, 1817) - Bull ray; Ratão-bispo (1), Raia (3)

Rhinoptera marginata (Geoffroy Saint-Hilaire, 1817) - Lusitanian cownose ray; Gavião-do-mar(1), (2) The presence of this species off the Azores needs further confirmation (Santos et al. 1997). The species $R$. marginata is regarded as being present off southern mainland Portugal, based on its geographical distribution (Ebert \& Stehmann 2013).

\section{Class Actinopterygii \\ Order Acipenseriformes \\ Family Acipenseridae}

* Acipenser sturio Linnaeus, 1758 - Sturgeon; Esturjão, Esturgião, Peixe-rei, Soilho or Sôlho-rei The last known records of sturgeon in Portugal refer to the presence of small specimens (20-30 cm long), during the early 1980s, in the lower Guadiana (Almaça 1988). The species is considered extinct (Rogado et al. 2005). In Portugal there are specimens of $A$. sturio only in museums and in captivity. For these reasons, $A$. sturio was not counted in the total number of species in the current list.

Order Elopiformes

Family Megalopidae

$\diamond \square$ Megalops atlanticus Valenciennes, 1847 - Trapon; Trapão-do-Atlântico(1), Trapão(1) (2), Peixeprata (2), (3)

Order Albuliformes

Family Halosauridae

$\square \quad$ Aldrovandia affinis (Günther, 1877) - No common name; (1) (2) (3)

Aldrovandia gracilis Goode \& Bean, 1896 - No common name; (3) 
This species occurs off Madeira, in the PECS area (Josephine Bank), BMNH, No. 1995.8.4.67-69, 14.5177 W, $36.7683^{\circ} \mathrm{N}, 12$ Apr. 1972.

Aldrovandia oleosa Sulak, 1977 - No common name; (2)

The presence of the species A. oleosa is reported off the Azores, in the PECS area (Atlantis Seamout); (Shcherbachev et al. 1985) AMK 4 - Academician Mstislav Keldysh, FISH 1556546, 29.9800 W, 34.4500 $\mathrm{N}$, depth 1240-1320 m.

$\square \quad$ Aldrovandia phalacra (Vaillant, 1888) - Hawaiian halosaurid fish; (1) (2)

Aldrovandia rostrata (Günther, 1878) - No common name; (2)

The occurrence of $A$. rostrata is reported off the Azores, in the PECS area (Great Meteor Tablemount), SDSC, No. $15910,28.2833^{\circ} \mathrm{W}, 36.8500^{\circ} \mathrm{N}$; FISH $1556510,28.2800^{\circ} \mathrm{W}, 29.8500^{\circ} \mathrm{N}$ (Shcherbachev et al. 1985).

Halosauropsis macrochir (Günther, 1878) - Abyssal halosaur; (1) (2) (3)

$\square \quad$ Halosaurus jonhsonianus Vaillant, 1888 - Halosaur; (1) (2)

* Halosaurus ovenii Johnson, 1864 - Halosaur; (1) (2) (3)

\section{Family Notacanthidae}

Leptocephalus giganteus Castle, 1959 - No common name; (1) (3)

According to Froese \& Pauly (2012), Coloconger giganteus (Castle, 1959) is a senior synonym of $L$. giganteus. The WoRMS Editorial Board (2013) indicates C. giganteus as an accepted species. The presence of C. giganteus is reported in the Madeiran EEZ (40 NM south of Madeira Is.), BMNH 1994.11.1.3922, 17.0 ${ }^{\circ}$ W, 32. $0^{\circ} \mathrm{N}$, and off mainland Portugal EEZ (16 NM NE of the Carlos Ribeiro Gap), BMNH. 1994.11.1.3931, $10.0^{\circ} \mathrm{W}, 32.0^{\circ} \mathrm{N}$. According to Smith (1989), L. giganteus is probably the larva of a Notacanthus species. Moser \& Charter (1996) indicated it as a larva of Notacanthus chemitzii Bloch, 1788. According to Eschmeyer (2013), L. giganteus is uncertain in the family Notacanthidae. This record should be considered provisional and confirmation is still needed.

* Notacanthus bonaparte Risso, 1840 - Shortfin spiny eel; (1) (2) (3)

The occurrence of this species is reported off the Azores, in the PECS area (113 NM and 152 NM WNW of Freen Trough), MAR-ECO, No. 4768, $29.5359^{\circ} \mathrm{W}, 42.9329^{\circ} \mathrm{N}, 2004.07 .09$; MAR-ECO, No. 6241, $28.5520^{\circ}$ $\mathrm{W}, 43.0340^{\circ} \mathrm{N}, 2004$.

* Notacanthus chemnitzii Bloch, 1788 - Spiny eel; (1) (3)

Polyacanthonotus africanus (Gilchrist \& von Bonde, 1924) - No common name; (2)

Polyacanthonotus challengeri (Vaillant, 1888) - No common name; (2) (3)

Polyacanthonotus rissoanus (De Filippi \& Verany, 1857) - Smallmouth spiny eel; (1) (2)

Order Anguilliformes

Family Anguillidae

* $\quad \square$ Anguilla anguilla (Linnaeus, 1758) - European eel; Enguia-europeia (1), Eiró(1) (2) (3), Iró (3) Anguilla rostrata (Lesueur, 1817) - American eel; Enguia-americana(2)

Family Chlopsidae

$\square \quad$ Chlopsis bicolor Rafinesque, 1810 - Bicouloured false moray; Congrinho-bicolor(2)

Family Muraenidae

According to Smith (2012) the classification of moray eels is still a work in progress, and has not yet resulted in a stable phylogeny. The family is imperfectly known since the definitions and boundaries of genera are still uncertain. Moreover, new species are continually being discovered and described.

Anarchias euryurus (Lea, 1913) - No common name; Peixe-lobo(2), (3)

$\square \quad$ Anarchias longicauda (Peter, 1877) - Bluenose moray; (3) 
Anarchias similis (Lea, 1913) - Pygmy moray; (2)

$\square \quad$ Enchelycore anatina (Lowe, 1838) - Fangtooth moray; Moreia-víbora or Víbora(2), Moreiaserpente (3)

Gymnothorax afer Bloch, 1795 - Dark moray; Moreão-escuro, (2)

The presence of this species off the Azores needs verification (Santos et al. 1997).

$\square \quad$ Gymnothorax bacalladoi Böhlke \& Brito, 1987 - Bacallado's moray; (3)

$\square \quad$ Gymnothorax maderensis (Johnson, 1862) - Sharktooh moray; (2), Moreia-da-Madeira or Moreão(3)

$\diamond \quad$ Gymnothorax miliaris (Kaup, 1856) - Goldentail moray; Moreia-dourada, (2)

$\square \quad$ Gymnothorax polygonius Poey, 1875 - Polygon moray; Moreão (3)

$\square \quad$ Gymnothorax unicolor (Delaroche, 1809) - Brown moray; Moreão-castanho (1), Moreão or Moreiacastanha (2), Moreão (3)

$\square \quad$ Gymnothorax vicinus (Castelnau, 1855) - Purplemouth moray; Moreão-amarelo, (2) (3)

$\square \quad$ Muraena augusti (Kaup, 1856) - No common name; Moreia-preta (2) (3), Moreão (2)

* $\square$ Muraena helena Linnaeus, 1758 - Mediterranean moray; Moreia (1) (2) (3), Moreia-pintada(2)

Family Synaphobranchidae

․ Dysomma brevirostre (Facciolà, 1887) - Pignosed arrowtooth eel; (1) (3)

Dysommina proboscideus (Lea, 1913) - No common name; (2)

Histiobranchus australis (Regan, 1913) - No common name; (2) (3)

Histiobranchus bathybius (Günther, 1877) - Deep-water arrowtooth eel; (1) (3), Moreão(2)

$\square \quad$ Ilyophis blachei Saldanha \& Merrett, 1982 - No common name; (2)

Ilyophis brunneus Gilbert, 1891 - Muddy arrowtooth eel; (2) (3)

$\square \quad$ Simenchelys parasitica Gill, 1879 - Snubnosed eel; Fluta(1), (2) (3)

- Synaphobranchus affinis Günther, 1877 - No common name; (1), Moreão-do-golfo (2), (3)

$\square \quad$ Synaphobranchus brevidorsalis Günther, 1887 - Shortdorsal cutthroat eel; (2)

Synaphobranchus dolichorhynchus (Lea, 1913) - No common name; (2)

This species is reported to occur off the Azores, in the PECS area (85 NM northwest of the Marsala Seamount), Scientific results of the Michael Sars North Atlantic Deep-Sea Expedition 1910, Sta. 53, 33.0166 $\mathrm{W}, 34.9833^{\circ}$ N, depth $150 \mathrm{~m}$. Known from larval specimens, adults not known (Eschmeyer 2013).

* $\square$ Synaphobranchus kaupii Johnson, 1862 - Kaup's arrowtooth eel; Moreão-de-natura (1) (3), Congrinho or Moreão(2), Moreia-de-natura (3)

Family Ophichthidae

Apterichtus anguiformis (Peters, 1877) - Slender finless eel; (3)

$\square \quad$ Aptherichthus caecus (Linnaeus, 1758) - European finless eel; Congrinho-da-areia(2), (3)

* Dalophis imberbis (Delaroche, 1809) - Armless snake eel; (1)

Echelus myrus (Linnaeus, 1758) - Painted eel; Cobra-de-orelhas (1), (3)

Myrophis plumbeus (Cope, 1871) - No common name; (2)

The presence of M. plumbeus is reported off the Azores, in the PECS area (155 NM southwest of the Georgiy

Zima Seamount), BMNH, No. 1994.11.1.1413, 20.3016 $\mathrm{W}, 39.8900^{\circ} \mathrm{N}$.

* $\square$ Ophisurus serpens (Linnaeus, 1758) - Serpent eel; Cobra-do-mar(1), (3)

Family Derichthyidae

Derichthys serpentinus Gill, 1884 - Narrownecked oceanic eel; (1) (2)

Nessorhamphus ingolfianus (Schmidt, 1912) - Duckbill oceanic eel; (1) (2) (3) 
Family Nemichthyidae

Avocettina infans (Günther, 1878) - Avocet snipp eel; Cobra-centopeia (2) (3)

Labichthys carinatus Gill \& Ryder, 1883 - No common name; (2)

The species L. carinatus is reported to occur to the south of the Azores, in the PECS area (46 NM SSW of the Oceanographer Fracture Zone, 62 NM SSW of the Konstantinov Ridge and 114 NM south of the Lucky Strike Seamount), MAR-ECO HamPelFish, No. $34845372,32.0169^{\circ}$ W, $35.4000^{\circ}$ N, 24 Apr. 1979; BMNH, No. $1994.11 .1 .1493,31.5000^{\circ} \mathrm{W}, 32.5450^{\circ} \mathrm{N}$; MAR-ECO HamPelFish, No. $34844931,35.4830^{\circ} \mathrm{W}, 34.3499^{\circ}$ N, 28 Apr. 1979.

Nemichthys curvirostris (Strömman, 1896) - Boxer snipe eel; (2) (3)

$* \square$ Nemichthys scolopaceus Richardson, 1848 - Slender snipe eel; Cobra-de-bico(1), (2) (3)

\section{Family Congridae}

$\square \quad$ Ariosoma balearicum (Delaroche, 1809) - Bandtooth conger; Congro-das-Baleares (1), (2) (3)

Bathyuroconger vicinus (Vaillant, 1888) - Large-toothed conger; (1)

* $\square$ Conger conger (Linnaeus, 1758) - European conger; Congro (1) (2) (3)

Conger triporiceps Kanazawa, 1958 - Manytooth conger; (3)

$\square \quad$ Gnathophis codoniphorus Maul, 1972 - No common name; (2)

The species G. codoniphorus is regarded as being present off the Azores (Maul 1972; Bauchot \& Saldanha 1986), in the PECS area (Great Meteor Tablemount), SDSC, No. 22978, 28.3966 ${ }^{\circ} \mathrm{W}, 29.8366^{\circ} \mathrm{N}, 1967.07 .22$; SDSC, No. 22979, 28.6667 $\mathrm{W}, 30.0499^{\circ} \mathrm{N}, 25$ Jul. 1967.

* $\square$ Gnathophis mystax (Delaroche, 1809) - Thinlip conger; Coreano(1), (2) (3)

$\square \quad$ Heteroconger longissimus Günther, 1870 - Garden eel; Enguia-de-jardim(3)

Paraconger macrops (Günther, 1870) - Blackspot conger; Congro-da-areia or Ortiga (2), Congrode-natura (3)

Pseudophichthys splendens (Lea, 1913) - Purplemouthed conger; (2)

Family Nettastomatidae

$* \diamond \square$ Facciolella oxyrhyncha (Bellotti, 1883) - Facciola's sorcerer; Cobra-cabeça-de-pato (1), (2) (3)

* Nettastoma melanurum Rafinesque, 1810 - Blackfin sorcerer; Cobra-bico-de-pato (1), (2) (3)

* Venefica proboscidea (Vaillant, 1888) - Whipsnout sorcerer; (1)

Family Serrivomeridae

$\square \quad$ Serrivomer beanii Gill \& Ryder, 1883 - Bean's sawtoothed eel; Cobra-couraça (1), (2) (3)

Serrivomer brevidentatus Roule \& Bertin, 1929 - Black sawtoothed eel; (1) (2) (3)

Serrivomer lanceolatoides (Schmidt, 1916) - Short-tooth sawpalate; (2)

Order Saccopharyngiformes

Family Cyematidae

Cyema atrum Günther, 1878 - Bobtail eel; Cobra-de-leme(1), (2) (3)

Family Saccopharyngidae

* Saccopharynx ampullaceus (Harwood, 1827) - Gluper eel; Enguia-pelicano (1), (2) (3) Saccopharynx harrisoni Beebe, 1932 - No common name; (3) Saccopharynx hjorti Bertin, 1938 - No common name; (2) 
This species is present off the Azores (86 NM northeast of the Marsala Seamount), and it is also regarded as being present off the Azores by Arruda (1997), although outside its EEZ. URNM, ZMB 6041, 33.0166 W, $34.9833^{\circ} \mathrm{N}, 9$ Jun. 1910.

Saccopharynx paucovertebratis Nielsen \& Bertelsen, 1985 - No common name; (3)

Saccopharynx ramosus Nielsen \& Bertelsen, 1985 - No common name; (2) (3)

The species $S$. ramosus is reported in the south of the Azores, but outside the EEZ ( 90 NM east of the Great Meteor Tablemount), Holotype, BMNH, No. 1983.11.19.2, $26.7166^{\circ} \mathrm{W}, 30.3066^{\circ} \mathrm{N}$.

Saccopharynx thalassa Nielsen \& Bertelsen, 1985 - No common name; (3)

Saccopharynx trilobatus Nielsen \& Bertelsen, 1985 - No common name; (2)

The species S. trilobatus is reported to occur southwest of the Azores (Arruda, 1997), in the PECS area (75

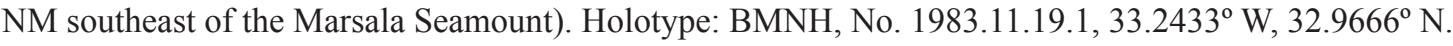

Family Eurypharyngidae

Eurypharynx pelecanoides Vaillant, 1882 - Pelican eel; Peixe-pelicano(1),(2) (3)

Family Monognathidae

The species Monognathus jesperseni Bertin, 1936 is regarded as being occurring off Portugal. One specimen was caught outside the Portuguese EEZ and also outside the proposed extension of the Portuguese continental shelf $\left(17.0016^{\circ} \mathrm{W}, 41.8630^{\circ} \mathrm{N}\right.$, Discovery Stn. 9801 \# 82(9)). For these reasons, M. jesperseni was not included in the total number of species in the current list. The specimen is deposited in the Natural History Museum, BMNH 1987.2.3.2.

Monognathus bertini Bertelsen \& Nielsen, 1987 - No common name; (1)

Monognathus boehlkei Bertelsen \& Nielsen, 1987 - No common name; (3)

Monognathus herringi Bertelsen \& Nielsen, 1987 - No common name; (3)

Monognathus nigeli Bertelsen \& Nielsen, 1987 - No common name; (2)

The species M. nigeli is reported to occur in the south of the Azores (Quéro et al. 2003), but outside EEZ (85

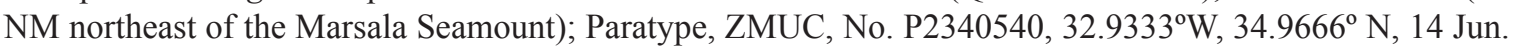
1981.

Order Clupeiformes

Family Engraulidae

* $\square$ Engraulis encrasicolus (Linnaeus, 1758) - European anchovy; Biqueirão (1), (2) (3)

Family Clupeidae

* Alosa alosa (Linnaeus, 1758) - Allis shad; Sável11

* Alosa fallax (Lacepède, 1803) - Twaite shad; Savelha (1), (3)

* $\square$ Sardina pilchardus (Walbaum, 1792) - European pilchard; Sardinha (1) (2) (3), Petinga (2)

* Sardinella aurita Valenciennes, 1847 - Round sardinella; Sardinela-lombuda (1), (3)

$\square \quad$ Sardinella maderensis (Lowe, 1838) - Madeiran sardinella; Sardinela-da-Madeira or Sardinelapalheta (1), Arenque (2) (3)

* Sprattus sprattus (Linnaeus, 1758) - European sprat; Espadilha (1), (2)

Order Argentiniformes

Family Argentinidae

* Argentina sphyraena Linnaeus, 1758 - Argentine; Argentina-branca (1), (2) (3) 
The occurrence of this species is reported off Madeira, in the PECS area (Josephine Bank), FISH 1556601 (Shcherbachev et al. 1985).

$\square \quad$ Glossanodon leioglossus (Valenciennes, 1848) - Smalltoothed argentine; Argentina-dourada (1), (2) (3)

The species G. leioglossus is reported to occur off the Azores, in the PECS area (Great Meteor Tablemount), SDSC, No. 22959, $28.4833^{\circ} \mathrm{W}, 29.8258^{\circ} \mathrm{N}, 18$ Jul. 1967; SDSC, No. 22960, $28.6399^{\circ} \mathrm{W}, 30.0849^{\circ} \mathrm{N}, 26 \mathrm{Jul}$. 1967.

\section{Family Opisthoproctidae}

Bathylychnops brachyrhynchus (Parr, 1937) - No common name; (2) The presence of the species B. brachyrhynchus is reported off the Azores, in the PECS area (62 NM north of the Kings Trough), ISH, No. 2638-1979, 22.2670 W, 44.9000 N, 5 Mar. 1979.

Bathylychnops exilis Cohen, 1958 - Javelin spookfish; (2)

Dolichopteryx longipes (Vaillant, 1888) - Brown-snout spookfish; (2) (3)

Opisthoproctus grimaldii Zugmayer, 1911 - Mirrorbelly; (1), Peixe-sola (2), (3)

Opisthoproctus soleatus Vaillant, 1888 - Barrel-eye; (1), Peixe-sola (2), (3)

$\square \quad$ Rhynchohyalus natalensis (Gilchrist \& von Bonde, 1924) - No common name; (2) (3)

The presence of this species is reported to the south and west of the Azores, in the PECS area (61 NM northeast of the Albany Seamount, 45 NM southwest of the Oceanographer Fracture Zone and 105 NM northwest of the Atlantis Seamount); MCZ, No. 66429, $35.9666^{\circ}$ W, $39.5000^{\circ}$ N, 27 Sep. 1964; ISH, No.

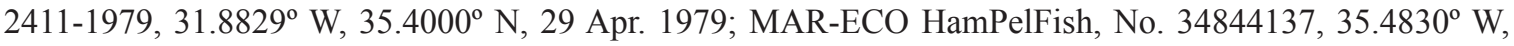
$34.3499^{\circ} \mathrm{N}, 28$ Apr. 1979.

Family Microstomatidae

Bathylagichthys greyae (Cohen, 1958) - Grey's deepsea smelt; (1) (2) (3) Bathylagus euryops Goode \& Bean, 1896 - Goiter blacksmelt; (1) (2) (3)

- Dolicholagus longirostris (Maul, 1948) - Longsnout blacksmelt; (1) (2) (3) Melanolagus bericoides (Borodin, 1929) - No common name; (1) (2) (3)

$\square \quad$ Microstoma microstoma (Risso, 1810) - Slender argentine; (2) (3) Nansenia atlantica Blache \& Rossignol, 1962 - No common name; (2) Nansenia groenlandica (Reinhardt, 1840) - Greenland argentine; (1) (2) Nansenia iberica Matallanas, 1985 - No common name; (2) The species $N$. iberica is present off the Azores, in the PECS area (106 NM SSW of the Olympus Knoll and at the Olympus Knoll), ISH, No. 967-1982, 28.4419 ${ }^{\circ}$ W, $43.6969^{\circ}$ N, 19 Jun. 1982; ISH, No. 465-1982, $27.6800^{\circ} \mathrm{W}, 45.4000^{\circ} \mathrm{N}, 11$ Jun. 1982.

Nansenia longicauda Kawaguchi \& Butler, 1984 - No common name; (3) Nansenia tenera Kawaguchi \& Butler, 1984 - No common name; (2)

\section{Family Platytroctidae}

Barbantus curvifrons (Roule \& Angel, 1931) - Palebelly searsid; (1) (2) (3)

The presence of $B$. curvifrons is reported to the south and northeast of the Azores in the PECS area (Plato Seamount and 157 NM southwest of the Georgiy Zima Seamount), SOC, Discovery No. 740618_8 FIS_066001, $20.1633^{\circ} \mathrm{W}, 40.0107^{\circ} \mathrm{N}, 3$ Oct. 1970; SOC, Discovery No. 1022202_85_FIS_066001, 30.0850 ${ }^{\circ}$ W, $33.0975^{\circ} \mathrm{N}, 29$ Oct. 1980.

Holtbyrnia anomala Krefft, 1980 - Bighead searsid; (2)

$\square \quad$ Holtbyrnia macrops Maul, 1957 - Bigeye searsid; (2) (3)

Maulisia argipalla Matsui \& Rosenblatt, 1979 - Palegold searsid; (2) (3)

- Maulisia mauli Parr, 1960 - Maul's searsid; (2) (3)

Maulisia microlepis Sazonov \& Golovan, 1976 - Smallscale searsid; (2) 
Mentodus facilis (Parr, 1951) - No common name; (3)

Mentodus mesalirus (Matsui \& Rosenblatt, 1987) - No common name; (2)

Mentodus rostratus Günther, 1878 - No common name; (2) (3)

Normichthys operosus Parr, 1951 - Multipore searsid; (1) (2)

Persparsia kopua (Phillipps, 1942) - No common name; (1)

Platytroctes apus Günther, 1878 - Legless searsid; (1) (2) (3)

Sagamichthys schnakenbecki (Krefft, 1953) - Schnakenbeck's searsid; (1) (2) (3)

- Searsia koefoedi Parr, 1937 - Koefoed's searsid; (1) (2) (3)

Family Bathylaconidae

Bathylaco nigricans Goode \& Bean, 1896 - Black warrior; (2) (3)

Herwigia kreffti (Nielsen \& Larsen, 1970) - Krefft's smooth-head; (2)

Family Alepocephalidae

Alepocephalus agassizii Goode \& Bean, 1883 - Agassiz' slickhead; (2)

Alepocephalus australis Barnard, 1923 - Small scaled brown slickhead; (2)

* $\square$ Alepocephalus bairdii Goode \& Bean, 1879 - Baird's slickhead; Celindra (1), (2) (3)

Alepocephalus productus Gill, 1883 - Smalleye smooth-head; (1) (2)

* Alepocephalus rostratus Risso, 1820 - Risso's smooth-head; Celindra(1) (3), (2)

Asquamiceps hjorti (Koefoed, 1927) - No common name; (2)

Asquamiceps velaris Zugmayer, 1911 - Fanfin smooth-head; (1)

* Bajacalifornia megalops (Lütken, 1898) - Bigeye smooth-head; (1) (2)

New record for the Portuguese mainland waters, the first specimen of B. megalops (Fig. 2), (280 mm TL and $104.68 \mathrm{~g}$ ) was caught by fishermen from Peniche, on the $7^{\text {th }}$ of April 2008 (39 $\left.30^{\prime} 3.23^{\prime \prime} \mathrm{N}, 9^{\circ} 30^{\prime} 32.37^{\prime \prime} \mathrm{W}\right)$. Bold Systems Sample ID-MLFPI111, available on the Barcode of Life Data Systems (BOLD; under the project titled "Fish of Portugal and Italy [MLFPI]": http://www.barcodinglife.org/).

Bathyprion danae Marshall, 1966 - Fangtooth smooth-head; (2) (3)

Bathytroctes macrolepis Günther, 1887 - Koefoed's smooth-head; (2)

Bathytroctes michaelsarsi Koefoed, 1927 - Michael Sars' smooth-head; (1) (2)

Bathytroctes microlepis Günther, 1878 - Smallscale smooth-head; (1) (2) (3)

Conocara fiolenti Sazonov \& Ivanov, 1979 - Fiolenti's smooth-head; (2)

Conocara macropterum (Vaillant, 1888) - Longfin smooth-head; (1), Celindra (2)

Conocara murrayi (Koefoed, 1927) - Murray's smooth-head; (1) (2)

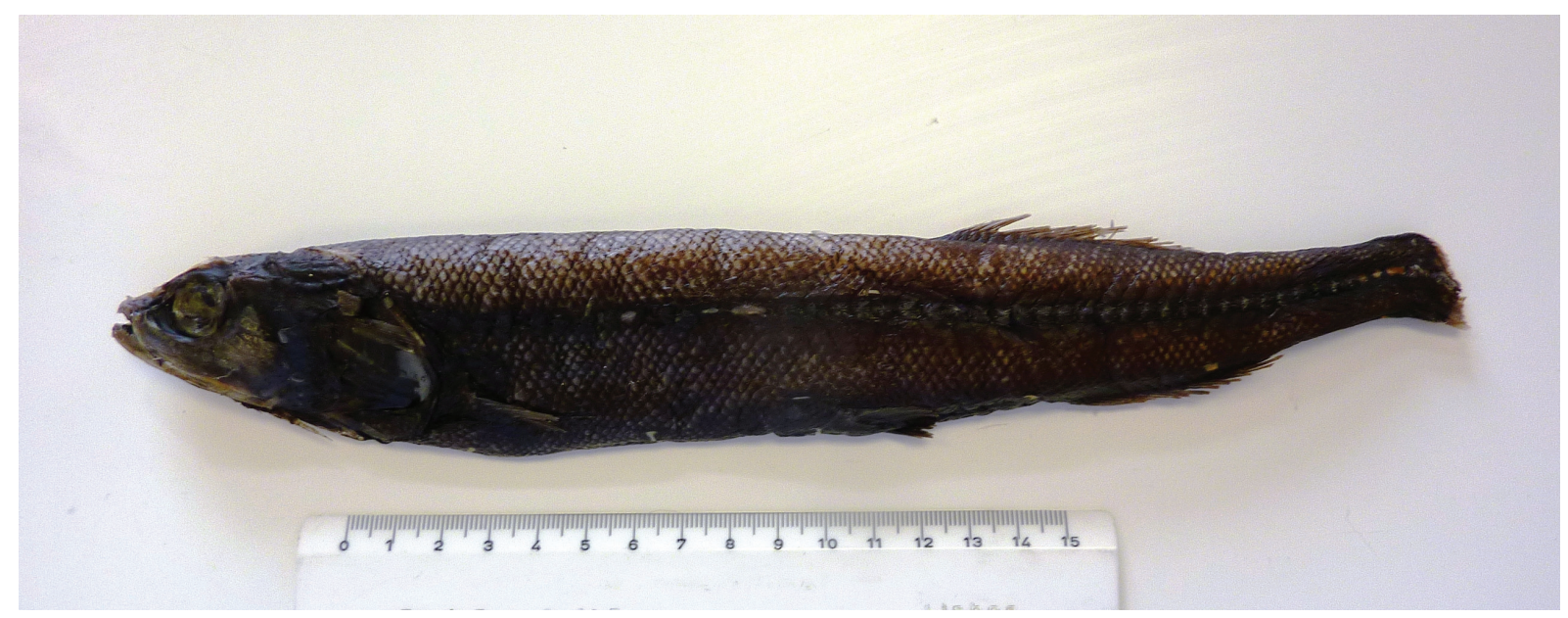

Fig. 2. Bajacalifornia megalops (Lütken, 1898). 
Conocara werneri Nybelin, 1947 - Werner's smooth-head; (1)

Einara edentula (Alcock, 1892) - Toothless smooth-head; (2) (3)

Einara macrolepis (Koefoed, 1927) - Loosescale smooth-head; (2) (3)

Leptochilichthys agassizii Garman, 1899 - Agassiz' smooth-head, (1)

Leptoderma macrophthalmum Byrkjedal, Poulsen \& Galbraith, 2011 - No common name; (2)

Mirognathus normani Parr, 1951 - Norman's smooth-head; (1)

Narcetes erimelas Alcock, 1890 - No common name; (2)

Narcetes stomias (Gilbert, 1890) - Blackhead salmon; (2)

Photostylus pycnopterus Beebe, 1933 - Starry smooth-head; (1) (2) (3)

Rinoctes nasutus (Koefoed, 1927) - Abyssal smooth-head; (2)

Rouleina attrita (Vaillant, 1888) - Softskin smooth-head; (1) (2)

$\square \quad$ Rouleina maderensis Maul, 1948 - Madeira smooth-head; (2) (3)

Talismania mekistonema Sulak, 1975 - Threadfin smooth-head; (2)

* Xenodermichthys copei (Gill, 1884) - Bluntsnout smooth-head; (1) (2) (3)

\section{Order Salmoniformes}

Family Salmonidae

Oncorhynchus mykiss (Walbaum, 1792) - Rainbow trout; Truta-arco-íris(1) (2) (3)

O. mykiss is an alien and invasive species introduced in Portugal. This species is primarily a freshwater fish, although sea-run populations, often known as steelhead, exist in some areas. However, populations of $O$. mykiss are capable of migrating to and surviving in the sea (Jonsson 2011).

* Salmo salar Linnaeus, 1758 - Atlantic salmon; Salmão-do-Atlântico or Salmão (1)

* Salmo trutta Linnaeus, 1758 - Sea trout; Truta-marisca (1)

Order Stomiiformes

Family Diplophidae

$\diamond \quad$ Diplophos taenia Günther, 1873 - No common name; (1) (2) (3)

The presence of this species is reported to the south and west of the Azores, in the PECS area (30 NM west of the Plato Seamount, $60 \mathrm{NM}$ south of the Pico Fracture Zone and $48 \mathrm{NM}$ north of the Oceanographer Fracture Zone), SOC, Discovery No. 1022207_84_FIS_218002, $30.0225^{\circ}$ W, $33.07830^{\circ}$ N, 30 Oct. 1980 ; SOC, Discovery No. 1022216_84_FIS_218002, $30.0357^{\circ} \mathrm{W}, 33.0900^{\circ} \mathrm{N}, 31$ Oct. 1980; SOC, Discovery No. 1022207_84_FIS_218002, 30.0225 $5^{\circ} \mathrm{W}, 33.0783^{\circ} \mathrm{N}, 30$ Oct. 1980; SOC, Discovery No. 1022221_84 FIS $218002, \overline{3} 0.0241^{\circ} \mathrm{W}, 33.0716^{\circ} \mathrm{N}, 30$ Oct. 1980 ; SOC, Discovery No. 1022217 85_FIS $218002,30.0625^{\circ}$ W, $33.0633^{\circ} \mathrm{N}, 31$ Oct. 1980 ; SOC, Discovery No. $1022218 \quad 86$ FIS $218002,30.0892^{\circ} \bar{W}, 33.0383^{\circ} \mathrm{N}, 31$ Oct. 1980; SOC, Discovery No. 102220585 FIS $218002,30.1291^{-} \mathrm{W}, 33.1458^{\circ} \mathrm{N}, 30$ Oct. 1980 ; SOC, Discovery No. 1022206 86_FIS_218002, $30.1566^{\circ} \mathrm{W}, 33.1724^{\circ} \mathrm{N}, 30$ Oct. 1980 ; MCZ, No. 62618, $35.0350^{\circ}$

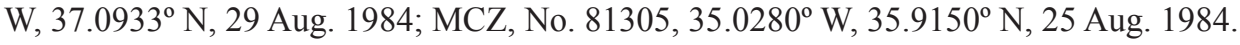

Family Gonostomatidae

Bonapartia pedaliota Goode \& Bean, 1896 - No common name; (1) (2) (3)

Cyclothone acclinidens Garman, 1899 - Benttooth bristlemouth; (1) (2) (3)

Cyclothone alba Brauer, 1906 - Bristlemouth; (2) (3)

* Cyclothone braueri Jespersen \& Tåning, 1926 - Garrick; (1) (2) (3)

Cyclothone livida Brauer, 1902 - Bristlemouth; (1) (2) (3)

Cyclothone microdon (Günther, 1878) - Bristlemouth; (1) (2) (3)

Cyclothone obscura Brauer, 1902 - No common name; (3)

Cyclothone pallida Brauer, 1902 - Bristlemouth; (1) (2) (3)

Cyclothone parapallida Badcock, 1982 - No common name; (3)

Cyclothone pseudopallida Mukhacheva, 1964 - Slender bristlemouth; (1) (2) (3) 
Cyclothone signata Garman, 1899 - Showy bristlemouth; (1) (2) Gonostoma atlanticum Norman, 1930 - No common name; (3)

* Gonostoma denudatum Rafinesque, 1810 - Bristlemouth; (1) (2) (3)

Gonostoma elongatum Günther, 1878 - Elongated bristlemouth fish; (1) (2) (3)

$\square \quad$ Manducus maderensis (Johnson, 1890) - No common name; (3)

Margrethia obtusirostra Jespersen \& Tåning, 1919 - Bristlemouth; (1) (2) (3)

Sigmops bathyphilus (Vaillant, 1884) - Bristlemouth; (1) (2) (3)

Family Sternoptychidae

$\square \quad$ Argyripnus atlanticus Maul, 1952 - No common name; (2) (3)

* Argyropelecus aculeatus Valenciennes, 1850 - Hatchetfish; Pai-velho (1) (2), (3) Argyropelecus affinis Garman, 1899 - No common name; Pai-velho(2) (3)

$\square \quad$ Argyropelecus gigas Norman, 1930 - Hatchetfish; (1), Pai-velho (2) (3)

* Argyropelecus hemigymnus Cocco, 1829 - Hatchetfish; Pai-velho(1) (2) (3)

* Argyropelecus olfersii (Cuvier, 1829) - Hatchetfish; (1), Pai-velho(2) (3) Argyropelecus sladeni Regan, 1908 - Sladen's hatchet fish; (2) (3) Maurolicus amethystinopunctatus Cocco, 1838 - Pearlsides; (1) (2) (3) Maurolicus muelleri (Gmelin, 1789) - Silvery lightfish; (1) (2) (3) Polyipnus polli Schultz, 1961 - No common name; (2) (3)

$\square \quad$ Sternoptyx diaphana Hermann, 1781 - Diaphanous hatchetfish; (1) (2), Pai-velho(2) (3) Sternoptyx pseudobscura Baird, 1971 - Highlight hatchetfish; (1) (2) (3) Valenciennellus tripunctulatus (Esmark, 1871) - No common name; (1) (2) (3)

Family Phosichthyidae

Ichthyococcus ovatus (Cocco, 1838) - Lightfish; (1) (2) (3)

Pollichthys mauli (Poll, 1953) - No common name; (2)

The presence of this species is recorded off the Azores, in the PECS area (208 NM southwest of the Georgiy Zima Seamount, 74 NM west of the Freen Trough, 70 NM southwest of the Albany Seamount, 61 NM southwest of the Hayes Fracture Zone and 110 NM south of the Marsala Seamount), MCZ, No. 140261, $20.7333^{\circ} \mathrm{W}, 41.0833^{\circ} \mathrm{N}$, 24 Jul. 1972; MAR-ECO 2004, No. 33278599, $27.7000^{\circ} \mathrm{W}, 42.8269^{\circ} \mathrm{N}$, 27 Jun. 2004; MCZ, No. $140405,38.0000^{\circ}$ W, $37.6666^{\circ}$ N, 8 Aug. 1931; MAR-ECO HamPelFish, No. 34850288 , $39.5670^{\circ} \mathrm{W}, 33.0169^{\circ} \mathrm{N}, 27$ Apr. 1979; SOC, Discovery No. 827000_8_FIS_644001, 34.3716 ${ }^{\circ} \mathrm{W}, 32.0575^{\circ}$ N, 1 Mar. 1973.

* Polymetme corythaeola (Alcock, 1898) - No common name; (1) (2) (3) P. corythaeola is reported to occur off the Azores, in the PECS area (Great Meteor Tablemount), SDSC, No. 22957, 28.6667 $\mathrm{W}, 30.0499^{\circ} \mathrm{N}, 25 \mathrm{Jul} .1967$.

Polymetme thaeocoryla Parin \& Borodulina, 1990 - No common name; (2) (3)

There are occurrences of the species P. thaeocoryla near Madeira, in the PECS area (Josephine Bank) (Froese \& Pauly 2012; Parin \& Borodulina 1990) and in the south of the Azores, in the PECS area (Great Meteor Tablemount) (Froese \& Pauly 2012; Parin \& Borodulina 1990).

$\square \quad$ Vinciguerria attenuata (Cocco, 1838) - Lightfish; (1) (2) (3)

Vinciguerria nimbaria (Jordan \& Williams, 1895) - Oceanic lightfish; (1) (2) (3)

$\square \quad$ Vinciguerria poweriae (Cocco, 1838) - Power's deep-water bristle-mouth fish; (1) (2) (3)

Family Stomiidae

Aristostomias grimaldii Zugmayer, 1913 - No common name; (2) (3)

Aristostomias lunifer Regan \& Trewavas, 1930 - No common name; (2) (3) 
The presence of this species is reported at the Azores, in the PECS area (60 NM northwest of the Marsala Seamount and 53 NM WSW of the Hayes Fracture Zone), ISH, No. 2049-1979, 35.4000 W, 34.3330 $\mathrm{N}, 26$

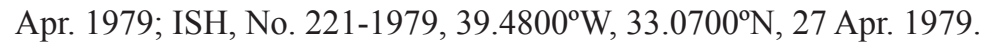

Aristostomias tittmanni Welsh, 1923 - Loosejaw; (2) (3)

Aristostomias xenostoma Regan \& Trewavas, 1930 - No common name; (2)

There are recorded occurrences of A. xenostoma off the Azores, in the PECS area (158 NM southwest of the Georgiy Zima Seamount and 13 specimens in the area between Konstantinov Ridge and South Atlantis Seamount), MNHN, Paratype No. $1938-0124,20.0000^{\circ} \mathrm{W}, 40.0000^{\circ} \mathrm{N}$.

Astronesthes atlanticus Parin \& Borodulina, 1996 - No common name; (3)

Astronesthes cyaneus (Brauer, 1902) - No common name; (2)

The presence of this species is reported off the Azores, in the PECS area (51 NM SSW of the Konstantinov Ridge), SOC, Discovery No. 1023214_85_FIS_013010,31.4666 W, 32.7649º N, 13 Nov. 1980.

$\square \quad$ Astronesthes gemmifer Goode \& Bean, 1896 - Snaggletooth; (1) (2) (3)

Astronesthes indicus Brauer, 1902 - No common name; (3)

Astronesthes leucopogon Regan \& Trewavas, 1929 - No common name; (1) (2) (3)

Astronesthes macropogon Goodyear \& Gibbs, 1970 - No common name; (3)

Astronesthes micropogon Goodyear \& Gibbs, 1970 - Snaggletooth; (2) (3)

$\square \quad$ Astronesthes neopogon Regan \& Trewavas, 1929 - Snaggletooth; (1) (2) (3)

Parin \& Borodulina (2000) indicated the species Astronesthes cyclophotus Regan \& Trewavas, 1929 as a synonym of A. neopogon. Porteiro (2005) indicated A. cyclophotus as a valid species, but the Catalog of Fishes (Eschmeyer 2013) and Fishbase (Froese \& Pauly 2012) continue to indicate the species A. cyclophotus as synonym of A. neopogon.

Astronesthes niger Richardson, 1845 - Snaggletooth; (1) (2) (3)

Astronesthes similus Parr, 1927 - No common name; (2)

This species occurs off the Azores, in the PECS area (63 NM southwest of the Hayes Fracture Zone), ISH,

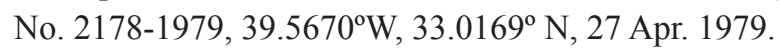

Astronesthes zharovi Parin \& Borodulina, 1998 - No common name; (2)

There are occurrences of this species off the Azores, in the PECS area (52 NM SSW of the Konstantinov Ridge), SOC, Discovery No. 1023215_86_FIS_013006, 31.4608 ${ }^{\circ}$ W, $32.7425^{\circ}$ N, 13 Nov. 1980; SOC, Discovery No. 1023217_86_FIS_013006, 31.4750 ${ }^{\circ} \mathrm{W}, 32.5180^{\circ} \mathrm{N}, 14$ Nov. 1980.

Bathophilus brevis Regan \& Trewavas, 1930 - No common name; (3)

B. brevis occurs to the southwest of the Azores, at $1000 \mathrm{~m}$ (Fowler 1936, without indicating the geographical coordinates).

Bathophilus digitatus (Welsh, 1923) - No common name; (2) (3)

Bathophilus longipinnis (Pappenheim, 1912) - No common name; (2) (3)

Bathophilus nigerrimus Giglioli, 1882 - Scaleless dragonfish; (2) (3)

Bathophilus pawneei Parr, 1927 - No common name; (2)

The species B. pawneei is reported to occur off the Azores, in the PECS area (Plato Seamount and 63 NM SSW of the Konstantinov Ridge), SOC, Discovery No. 1022207_84_FIS_067006, 30.0220 W, $33.0780^{\circ} \mathrm{N}$,

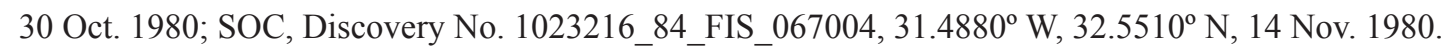

Bathophilus proximus Regan \& Trewavas, 1930 - No common name; (2)

This species is recorded from the Azores, in the PECS area (37 NM southwest of the Konstantinov Ridge), SOC, Discovery No. 1024104_84_FIS_067008, 31.8025으, $33.1858^{\circ} \mathrm{N}, 18$ Nov. 1980.

Bathophilus vaillanti (Zugmayer, 1911) - Scaleless dragonfish; (1) (2) (3)

Borostomias antarcticus (Lönnberg, 1905) - Snaggletooth; (1) (2)

Borostomias elucens (Brauer, 1906) - No common name; (2) (3)

B. elucens is recorded off the Azores, in the PECS area (Plato Seamount), SOC, Discovery No. 1022203_86 FIS_076001, $30.1158^{\circ} \mathrm{W}, 33.1208^{\circ} \mathrm{N}, 29$ Oct. 1980.

Borostomias mononema (Regan \& Trewavas, 1929) - No common name; (3)

Chauliodus danae Regan \& Trewavas, 1929 - Dana viperfish; (1) (2) (3)

* Chauliodus sloani Bloch \& Schneider, 1801 - Sloane’s viperfish; Demónio or Peixe-demónio (1) (2) (3) 
Chirostomias pliopterus Regan \& Trewavas, 1930 - Scaleless dragonfish; (1) (2) (3)

$\square \quad$ Echiostoma barbatum Lowe, 1843 - No common name; (1) (2) (3)

Eustomias braueri Zugmayer, 1911 - No common name; (1) (2) (3)

Eustomias contiguus Gomon \& Gibbs, 1985 - No common name; (2) (3)

This species is recorded from the Azores, in the PECS area (59 NM northwest of the Marsala Seamount, 52 NM southwest and 87 NM northwest of the Hayes Fracture Zone), ZMH, Holotype No. 137943, 35.3670 W, $34.3499^{\circ}$ N; USNM, No. 322759, 35.4830 ${ }^{\circ}$ W, $34.3500^{\circ}$ N, 28 Apr. 1979; ZMH, Paratype No. 137942, $39.5666^{\circ} \mathrm{W}, 35.0333^{\circ} \mathrm{N}$; ZMH, Paratype No. $138424,39.4833^{\circ} \mathrm{W}, 33.0666^{\circ} \mathrm{N}$.

Eustomias dubius Parr, 1927 - No common name; (2)

This species is reported to occur off the Azores, in the PECS area (30 NM SSW of the Konstantinov Ridge),

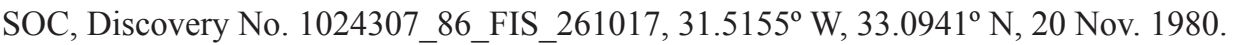

Eustomias enbarbatus Welsh, 1923 - No common name; (2)

The presence of E. enbarbatus is confirmed from the Azores, in the PECS area (40 NM southwest of the Oceanographer Fracture Zone), USNM, No. 379984, 35.4830 W, 34.3500 N, 28 Apr. 1979; USNM, No. $372074,35.3670^{\circ} \mathrm{W}, 34.3500^{\circ} \mathrm{N}, 28$ Apr. 1979.

Eustomias filifer (Gilchrist, 1906) - No common name; (2) (3)

There are records of E. filifer from the Azores, in the PECS area (53 NM NNW of the Maxwell Fracture Zone, Olympus Knoll and 46 NM southwest of the Oceanographer Fracture Zone), MAR-ECO - HamPelFish, No. $34847890,27.5330^{\circ} \mathrm{W}, 48.5880^{\circ} \mathrm{N}, 14$ Jun. 1982; MAR-ECO - HamPelFish, No. 34847888, $27.8029^{\circ} \mathrm{W}$, $45.6669^{\circ} \mathrm{N}$, 11 Jun. 1982; MAR-ECO - HamPelFish, No. 34847889, $27.6870^{\circ} \mathrm{W}, 45.3969^{\circ} \mathrm{N}, 11$ Jun. 1982; MAR-ECO - HamPelFish, No. 34847889, 27.6870 ${ }^{\circ}$ W, 45.3699 ${ }^{\circ}$ N, 11 Jun. 1982; USNM, No. 322960, $35.4830^{\circ} \mathrm{W}, 34.3499^{\circ} \mathrm{N}, 28$ Apr. 1979.

Eustomias fissibarbis (Pappenheim, 1912) - No common name; (2) (3)

The presence of this species is reported from the Azores, in the PECS area (47 NM southwest of the Oceanographer Fracture Zone), MAR-ECO - HamPelFish, No. 34847895, 35.4830 $\mathrm{W}, 34.3499^{\circ} \mathrm{N}, 28 \mathrm{Apr}$. 1979.

Eustomias furcifer Regan \& Trewavas, 1930 - Scaleless dragonfish; (2)

Eustomias lipochirus Regan \& Trewavas, 1930 - No common name; (2) (3)

E. lipochirus is reported to occur off the Azores, in the PECS area (56 NM southwest of the Hayes Fracture

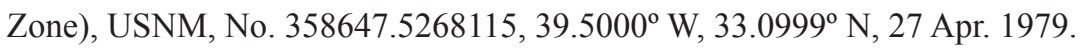

Eustomias longibarba Parr, 1927 - No common name; (2) (3)

E. longibarba is reported off the Azores, in the PECS area (45 NM southwest of the Oceanographer Fracture Zone), MAR-ECO - HamPelFish, No. 348447902, 35.4830 $\mathrm{W}, 34.3499^{\circ}$ N, 28 Apr. 1979.

Eustomias macronema Regan \& Trewavas, 1930 - No common name; (3)

Eustomias macrurus Regan \& Trewavas, 1930 - Yellowstem dragonfish; (2)

Eustomias monodactylus Regan \& Trewavas, 1930 - No common name; (2)

This species is recorded off the Azores, in the PECS area (60 NM southwest of the Hayes Fracture Zone), USNM, No. 322963, 39.5670 $\mathrm{W}, 33.0170^{\circ} \mathrm{N}, 27$ Apr. 1979.

Eustomias obscurus Vaillant, 1884 - Scaleless dragonfish; (1) (2) (3)

Eustomias parri Regan \& Trewavas, 1930 - No common name; (2)

Eustomias paucifilis Parr, 1927 - No common name.

The species E. paucifilis was regarded as being present to the southwest of the Azores, at a depth of $2000 \mathrm{~m}$ (Fowler 1936, without indication of the geographical coordinates). In later years, there was one record in the southwest of the Azores (Post 1987), but the specimen was caught outside the EEZ and outside the proposed extension of the Portuguese continental shelf. Because of uncertainties in its distribution, this species was not considered for the estimation of the total number of species in the checklist.

Eustomias radicifilis Borodin, 1930 - No common name; (2)

The species is reported off the Azores, in the PECS area (108 NM SSW of the Marsala Seamount), SOC, Discovery No. 827000_8_FIS_261016, 34.3716 W, 32.0575º N, 2 Mar. 1973.

Eustomias satterleei Beebe, 1933 - No common name; (2)

This species is recorded off the Azores, in the PECS area (44 NM southwest of the Oceanographer Fracture Zone), MAR-ECO - HamPelFish, No. 34849205, 35.4830 W, 34.3499º N, 28 Apr. 1979. 
Eustomias schmidti Regan \& Trewavas, 1930 - No common name; (2) (3)

There are records of the species E. schmidti off the Azores, in the PECS area (59 NM WSW of the Hayes Fracture Zone and $33 \mathrm{NM}$ SSW of the Konstantinov Ridge), USNM, No. $292889,39.5670^{\circ} \mathrm{W}, 33.0170^{\circ}$

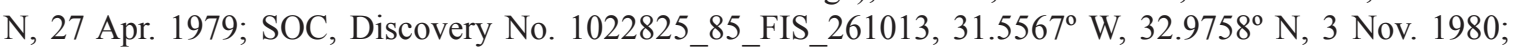
SOC, Discovery No. 1022824_84_FIS_261013, $31.5233^{\circ}$ W, $32.9400^{\circ} \mathrm{N}, 3$ Nov. 1980; SOC, Discovery No. 1022826 86_FIS_261013, 31.5900 W, 33.0141 ${ }^{\circ} \mathrm{N}, 3$ Nov. 1980.

Eustomias simplex Regan \& Trewavas, 1930 - No common name; (2) (3)

There are records of the species E. simplex to the south of the Azores, in the PECS area (43 NM southwest of the Oceanographer Fracture Zone and 35 NM southwest of the Konstantinov Ridge), USNM, No. 372019, $35.3670^{\circ} \mathrm{W}, 34.3500^{\circ} \mathrm{N}, 28$ Apr. 1979; USNM, No. 379335, 35.4830 $\mathrm{W}, 34.3500^{\circ} \mathrm{N}, 28$ Apr. 1979; SOC, Discovery No. 102280485 FIS_261010, $31.4583^{\circ}$ W, 33.0342 ${ }^{\circ}$ N, 1 Jan. 1980; SOC, Discovery No. 102280586 FIS $261010,31.4550^{\circ} \mathrm{W}, 33.0725^{\circ} \mathrm{N}, 1$ Nov. 1980.

Eustomias tetranema Zugmayer, 1913 - No common name; (2) (3)

Flagellostomias boureei (Zugmayer, 1913) - Scaleless dragonfish; (1) (2) (3)

Grammatostomias circularis Morrow, 1959 - No common name; (3)

Grammatostomias dentatus Goode \& Bean, 1896 - No common name; (1) (2) (3)

G. dentatus occurs off the Azores, in the PECS area (60 NM northwest of the Marsala Seamount), MAR-ECO

- HamPelFish, No. 34849221, 35.4830 W, 34.3499 N, 28 Apr. 1979.

Grammatostomias flagellibarba Holt \& Byrne, 1910 - No common name; (3)

Idiacanthus fasciola Peters, 1877 - Sawtailfish; (1) (2) (3)

Leptostomias gladiator (Zugmayer, 1911) - Scaleless dragonfish; (1) (2) (3)

Leptostomias haplocaulus Regan \& Trewavas, 1930 - No common name; (1) (2) (3)

Leptostomias longibarba Regan \& Trewavas, 1930 - No common name; (2)

$\square \quad$ Malacosteus niger Ayres, 1848 - Stoplight loosejaw; (1) (2) (3)

Melanostomias bartonbeani Parr, 1927 - Scaleless dragonfish; (1) (2) (3)

Melanostomias biseriatus Regan \& Trewavas, 1930 - No common name; (2) (3)

There are occurrences of the species M. biseriatus off the Azores, in the PECS area (39 NM southwest and 42 NM south of the Konstantinov Ridge), SOC, Discovery No. 1024117_84_FIS_518003, 31.8266 W, $33.1958^{\circ}$ N, 19 Nov. 1980; SOC, Discovery No. 1024405_84_FIS_518003, 31.2608 W, 32.8441 ${ }^{\circ}$ N, 21 Nov. 1980.

Melanostomias macrophotus Regan \& Trewavas, 1930 - No common name; (1) (2) (3)

Melanostomias melanopogon Regan \& Trewavas, 1930 - No common name; (2)

Melanostomias melanops Brauer, 1902 - No common name; (2) (3)

This species is recorded off the Azores, in the PECS area (Plato Seamount and 64 NM southwest of the Hayes Fracture Zone), USNM, No. 358822, $29.6000^{\circ}$ W, $33.0999^{\circ}$ N, 27 Apr. 1979; USNM, No. 358821, 39.567 W, $32.9830^{\circ}$ N, 27 Apr. 1979.

Melanostomias tentaculatus (Regan \& Trewavas, 1930) - No common name; (2) (3)

There are records of the species M. tentaculatus in the north and in the southwest of the Azores, in the PECS area (29 NM WNW of the Sherkis Seamount, 48 NM southwest of the Oceanographer Fracture Zone and 63 NM WSW of the Hayes Fracture Zone), MAR-ECO - HamPelFish, No. 34849776, 26.2469 ${ }^{\circ}$ W, $43.2420^{\circ}$ N, 9 Jun. 1982; USNM, No. 358831.5268546, 35.4000 W, $34.2999^{\circ}$ N, 28 Apr. 1979; USNM, No. $358662.5268130,39.5999^{\circ} \mathrm{W}, 33.0000^{\circ} \mathrm{N}, 27$ Apr. 1979.

Melanostomias valdiviae Brauer, 1902 - Valdivia black dragon fish; (1) (2) (3)

Neonesthes capensis (Gilchrist \& von Bonde, 1924) - Cape snaggletooth; (1) (2) (3)

Pachystomias microdon (Günther, 1878) - Smalltooth dragonfish; (1) (2)

Photonectes braueri (Zugmayer, 1913) - Scaleless dragonfish; (2) (3)

Photonectes dinema Regan \& Trewavas, 1930 - No common name; (2) (3)

Photonectes margarita (Goode \& Bean, 1896) - No common name; (2) (3)

Photonectes mirabilis Parr, 1927 - No common name; (2) (3)

The occurrence of this species is reported off the Azores, in the PECS area (58 NM WNW of the Marsala Seamount), MAR-ECO - HamPelFish, No. 34848521, 35.4000 W, 34.3330 N, 28 Apr. 1979.

Photonectes parvimanus Regan \& Trewavas, 1930 - No common name; (1) (2) (3) 
The species P. parvimanus is regarded as being present southwest of the Azores (Albuquerque 1954-1956) and there is one record off the Azores, in the PECS area (95 NM WNW of the Hyeres Seamount), MCZ, No. $132081,30.6166^{\circ} \mathrm{W}, 31.8333^{\circ} \mathrm{N}, 5$ Sep. 1973.

Photostomias atrox (Alcock, 1890) - No common name; (3)

Photostomias goodyeari Kenaley \& Hartel, 2005 - No common name; (2) (3)

Photostomias guernei Collett, 1889 - Loosejaw; (1) (2) (3)

Rhadinesthes decimus (Zugmayer, 1911) - Slender snaggletooth; (1) (2) (3)

Stomias boa boa (Risso, 1810) - Boa dragonfish; (1) (3)

Stomias boa ferox Reinhardt, 1842 - Scaly dragonfish; (1) (2) (3)

$\square \quad$ Stomias brevibarbatus Ege, 1918 - No common name; (1) (2) (3)

Stomias longibarbatus (Brauer, 1902) - No common name; (1) (2) (3)

Trigonolampa miriceps Regan \& Trewavas, 1930 - Threelight dragonfish; (2)

Order Ateleopodiformes

Family Ateleopodidae

Guentherus altivela Osório, 1917 - Jellynose; (1)

Order Aulopiformes

Family Aulopodidae

* $\square$ Aulopus filamentosus (Bloch, 1792) - Royal flagfin; Lagarto-do-mar(1) (3), Lagarto-do-alto or Peixelagarto (2), Lagarto (3)

Family Synodontidae

Synodus foetens (Linnaeus, 1766) - Inshore lizardfish; (2)

There are records of the species $S$. foetens off the Azores, in the PECS area (80 NM west of the Great Meteor Tablemount), ROM, No. 23913, 30.2500 W, 30.2916 N, 29 Jan. 1966.

Synodus myops (Forster, 1801) - Snakefish; Lagarto-focinho-rombo, (2)

* $\square$ Synodus saurus (Linnaeus, 1758) - Atlantic lizardfish; Lagarto-da-costa (1) (2) (3), Peixe-lagarto (2)

$\square \quad$ Synodus synodus (Linnaeus, 1758) - Diamond lizardfish; Lagarto-de-rolo (3)

Family Chlorophthalmidae

* Chlorophthalmus agassizi Bonaparte, 1840 - Shortnose greeneye; Olho-verde (1); Olho-vivo(2), (3)

Family Notosudidae

Ahliesaurus berryi Bertelsen, Krefft \& Marshall, 1976 - No common name; (1) (2) (3)

$\square \quad$ Scopelosaurus argenteus (Maul, 1954) - Waryfish; (1) (2) (3)

Scopelosaurus lepidus (Krefft \& Maul, 1955) - Waryfish; (1) (2) (3)

$\square \quad$ Scopelosaurus smithii Bean, 1925 - No common name; (2) (3)

Family Ipnopidae

Bathymicrops multispinis Nielsen \& Merrett, 1992 - No common name; (3)

There are occurrences of $B$. multispinis off Madeira in the PECS area (Madeira Plain), USNM, No. $344624.5250741,21.1250^{\circ} \mathrm{W}, 31.2483^{\circ} \mathrm{N}, 20$ Aug. 1990; BMNH, No. 1997.1.2.2, 21.1180 $\mathrm{W}, 31.2358^{\circ} \mathrm{N}$.

Bathymicrops regis Hjort \& Koefoed, 1912 - No common name; (2) (3)

There are records of this species to the south of the Azores, in the PECS area (15 NM west of the Great Meteor Tablemount), SDSC, No. 16042, 27.8999 $\mathrm{W}, 29.7999^{\circ}$ N, 29 Jun. 1982 (Shcherbachev et al. 1985). 
$\square \quad$ Bathypterois dubius Vaillant, 1888 - Spiderfish; (1), Peixe-tripé(2)

Bathypterois grallator (Goode \& Bean, 1886) - Tripodfish; (1), Peixe-tripé(2)

Bathypterois longipes Günther, 1878 - Abyssal spiderfish; (1), Peixe-tripé-abissal(2), (3)

Bathypterois phenax Parr, 1928 - Blackfin spiderfish; Peixe-tripé-de-barbatana-negra(2)

Bathytyphlops sewelli (Norman, 1939) - No common name; (2)

Family Scopelarchidae

Benthalbella infans Zugmayer, 1911 - Zugmayer's pearleye; (1) (2) (3)

Rosenblattichthys hubbsi Johnson, 1974 - Hubb's pearleye; (2)

The species R. hubbsi is reported off the Azores, in the PECS area (90 NM WNW of the Irving Seamount), MCZ, No. 52225, $30.6833^{\circ}$ W, $31.7333^{\circ}$ N, 6 Sep. 1973; MCZ, No. 52223, $30.6166^{\circ}$ W, 31.8333 N, 5 Sep. 1973.

$\square \quad$ Scopelarchus analis (Brauer, 1902) - Short fin pearleye; (1) (2) (3)

Scopelarchus guentheri Alcock, 1896 - Staring pearleye; (2)

Scopelarchus michaelsarsi Koefoed, 1955 - Bigfin pearleye; (2)

The species S. michaelsarsi is reported off the Azores, in the PECS area (34 NM southwest of the Konstantinov Ridge), MCZ, No. 71113, 30.6166 W, 31.8333 N, 5 Sep. 1973.

Family Evermannellidae

Coccorella atlantica (Parr, 1928) - Atlantic sabertooth; (2) (3)

Coccorella atrata (Alcock, 1894) - No common name; (2)

This species is reported off the Azores, in the PECS area (78 NM WSW of the Plato Seamount), SOC,

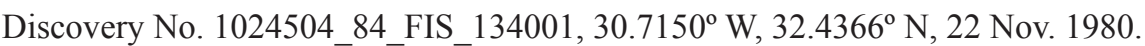

$\square \quad$ Evermannella balbo (Risso, 1820) - Balbo sabertooth; (1) (2) (3)

Evermannella melanoderma Parr, 1928 - India sabertooth; (1) (3)

This Atlantic species has the same English common name as E. indica Brauer, 1906. A revision of the genus Evermannella resulted in the distinction between the species (Swinney 1994). Therefore, all previous records of E. indica in the Atlantic are now known as E. melanoderna. In our opinion the common name should be modified.

\section{Family Alepisauridae}

Alepisaurus brevirostris Gibbs, 1960 - Short snouted lancetfish; Peixe-cavalo(2)

* $\square$ Alepisaurus ferox Lowe, 1833 - Long snouted lancetfish; Lírio-ferro (1), (2), Peixe-cavalo or Peixeágua (3)

Omosudis lowii Günther, 1887 - Omosudid; (1) (2) (3)

Family Paralepididae

- Anotopterus pharao Zugmayer, 1911 - Daggertooth; (1) (2) (3)

- Arctozenus risso (Bonaparte, 1840) - Spotted barracudina; (1) (2) (3)

Lestidiops affinis (Ege, 1930) - Barracudina; Barracudina (2) (3)

$\square \quad$ Lestidiops jayakari pseudosphyraenoides (Ege, 1918) - No common name; (1), Barracudina (2) (3) Lestidiops similis (Ege, 1933) - No common name; (2)

There are records of this species to the north and in the south of the Azores, in the PECS area (five specimens in the Mid-Atlantic Ridge between northwest of the Sherkis Seamount and NNW of the Maxwell Fracture Zone; 81 NM SSW of the Crumb Seamount and 46 NM southwest of the Oceanographer Fracture Zone), MARECO - HamPelFish, No. 34847972, $27.8020^{\circ} \mathrm{W}, 48.1500^{\circ}$ N, 14 Jun. 1982; MAR-ECO - HamPelFish, No. $348447970,27.3320^{\circ} \mathrm{W}, 47.0449^{\circ} \mathrm{N}, 12$ Jun. 1982; MAR-ECO - HamPelFish, No. 34847971, $27.2380^{\circ} \mathrm{W}$, $46.4900^{\circ}$ N, 12 Jun. 1982; MAR-ECO - HamPelFish, No. 34847969, 27.6870 W, $45.3969^{\circ}$ N, 11 Jun. 1982; 
MAR-ECO - HamPelFish, No. 34847968; $28.5820^{\circ} \mathrm{W}, 43.6049^{\circ}$ N, 10 Jun. 1982; MAR-ECO - HamPelFish, No. $34847964,23.4920^{\circ} \mathrm{W}, 42.1080^{\circ} \mathrm{N}, 2$ May 1979; MAR-ECO - HamPelFish, No. 34847552, $35.4830^{\circ} \mathrm{W}$, $34.3499^{\circ} \mathrm{N}, 28$ Apr. 1979.

Lestidiops sphyrenoides (Risso, 1820) - Barracudina; (1), Barracudina (2), (3)

$\square \quad$ Macroparalepis affinis Ege, 1933 - Barracudina; Barracudina(2), (3)

Macroparalepis brevis Ege, 1933 - No common name; (2)

$\square \quad$ Macroparalepis nigra (Maul, 1965) - No common name; (3)

$\square \quad$ Magnisudis atlantica (Krøyer, 1868) - Duckbill barracudine; (1), Barracudina(2), (3)

$\square \quad$ Paralepis brevirostris (Parr, 1928) - No common name; (2) (3)

$\square \quad$ Paralepis coregonoides Risso, 1820 - Lancet fish; (1), Barracudina(2), (3)

Paralepis elongata (Brauer, 1906) - No common name; (2)

The species P. elongata is reported to occur off the Azores, in the PECS area (105 NM SSW of the Konstantinov Ridge), SOC, Discovery No. 1023306_86_FIS_647004, 31.5842 ${ }^{\circ}$ W, $31.8633^{\circ}$ N, 14 Nov. 1980.

Paralepis speciosa Bellotti, 1878 - No common name; Barracudina(2), (3)

Sudis atrox Rofen, 1963 - Fierce pike smelt; (2)

There are records of this species in the south of the Azores, in the PECS area (80 NM west of the Hyères Seamount), MCZ, No. $67382,30.6166^{\circ} \mathrm{W}, 31.8333^{\circ} \mathrm{N}, 5$ Sep. 1973 ; MCZ, No. $67361,30.6833^{\circ} \mathrm{W}, 31.7333^{\circ}$ N, 6 Sep. 1973.

- Sudis hyalina Rafinesque, 1810 - Barracudina; (1), Barracudina (2), (3)

$\square \quad$ Uncisudis longirostra Maul, 1956 - No common name; (3)

Uncisudis quadrimaculata (Post, 1969) - Barracudina; Barracudina(2)

Family Bathysauridae

Bathysaurus ferox Günther, 1878 - Deep-sea lizardfish; (2) (3)

Bathysaurus mollis Günther, 1878 - No common name; (1) (3), Lagarto-da-costa or Peixe-lagarto(2)

Order Myctophiformes

Family Neoscopelidae

$\square \quad$ Neoscopelus macrolepidotus Johnson, 1863 - Large-scaled lantern fish; (2), Lanterna-vermelha(3) Neoscopelus microchir Matsubara, 1943 - No common name; (1) (3)

Scopelengys tristis Alcock, 1890 - Pacific blackchin; (3)

Family Myctophidae

Benthosema glaciale (Reinhardt, 1837) - Glacier lantern fish; Romeirinho (1), Divertido, Escolarinho or Lobisomem (2), (3)

Benthosema suborbitale (Gilbert, 1913) - Smallfin lanternfish; Romeirinho(1), Divertido, Escolarinho or Lobisomem (2), (3)

Bolinichthys indicus (Nafpaktitis \& Nafpaktitis, 1969) - Lanternfish; (1), Divertido, Escolarinho or Lobisomem (2), (3)

Bolinichthys longipes (Brauer, 1906) - No common name; (3)

Brauer (1906) described a new species as Myctophum (Lampanyctus) longipes, caught at $75 \mathrm{NM}$ southeast of Madeira (Valdivia, Stat. $26-31^{\circ} 59.050^{\prime} \mathrm{N}, 15^{\circ} 5.000^{\prime} \mathrm{W}$ ) during the German Deep-Sea Expedition of the "Valdivia", during 1898-1899. According to Haulley \& Duhamel (2009), the type specimen caught near Madeira (Myctophum longipes Brauer, 1906, accepted as B. longipes) is apparently referable to B. indicus. The occurrence of this species in Madeira, however, is questionable.

Bolinichthys photothorax (Parr, 1928) - Spurcheek lanternfish; (3)

The species B. photothorax is regarded as being present off Madeira (Haulley \& Duhamel 2009) (eastern Atlantic between $\left.33^{\circ} \mathrm{N}-35^{\circ} \mathrm{S}\right)$.

Bolinichthys pyrsobolus (Alcock, 1890) - No common name; (1) 
Bolinichthys supralateralis (Parr, 1928) - No common name; (2) (3)

Centrobranchus nigroocellatus (Günther, 1873) - No common name; Romeirinho(2), (3)

$\square \quad$ Ceratoscopelus maderensis (Lowe, 1839) - Madeira lantern fish; Peixinho-preto(1), Divertido, Escolarinho or Lobisomem (2), Romeirinho (3)

$\diamond \square$ Ceratoscopelus warmingii (Lütken, 1892) - Lanternfish; (1), Divertido, Escolarinho or Lobisomem (2), Romeirinho (3)

Diaphus adenomus Gilbert, 1905 - No common name; (3)

Diaphus bertelseni Nafpaktitis, 1966 - No common name; (1) (2), Romeirinho(3)

$\diamond$ Diaphus brachycephalus Tåning, 1928 - Short-headed lantern fish; (1), Divertido, Escolarinho or Lobisomem (2), Romeirinho (3)

$\diamond \square$ Diaphus dumerilii (Bleeker, 1856) - Lantern fish; Ferreiro(1), Divertido, Escolarinho or Lobisomem (2), (3)

Diaphus effulgens (Goode \& Bean, 1896) - Headlight fish; Divertido, Escolarinho or Lobisomem (2), (3)

Diaphus holti Tåning, 1918 - Small lantern fish; (1), Divertido, Escolarinho or Lobisomem (2), Romeirinho(3)

Diaphus lucidus (Goode \& Bean, 1896) - No common name; (2), Romeirinho (3)

There are records of this species to the south of the Azores, in the PECS area (37 NM and 103 NM SSW of the Konstantinov Ridge, 109 NM south of the Marsala Seamount, 43 NM SSW of the Oceanographer Fracture Zone and 58 NM southwest of the Hayes Fracture Zone), SOC, Discovery No. 1022804_85_FIS_214006, $314583^{\circ} \mathrm{W}, 33.0342^{\circ} \mathrm{N}, 1$ Nov. 1980 ; SOC, Discovery No. 1024405 84_FIS $214006,31.2608^{\circ} \mathrm{W}, 32,8441^{\circ}$ N, 21 Nov. 1980; SOC, Discovery No. 1024401_84_FIS_214006, $31.2608^{\circ}$ W, 32.8549 ${ }^{\circ}$ N, 20 Nov. 1980; SOC, Discovery No. 1023305 85_FIS_214006, $31.5675^{\circ} \mathrm{W}, 31.8924^{\circ} \mathrm{N}, 14$ Nov. 1980 ; SOC, Discovery No. 827000_8_FIS_214006, $34.3716^{\circ} \mathrm{W}, 32.0575^{\circ}$ N, 2 Mar. 1973; MAR-ECO - HamPelFish, No. 34846583, $35.3670^{\circ} \mathrm{W}, 34.3499^{\circ} \mathrm{N}, 28$ Apr. 1979; MAR-ECO - HamPelFish, No. $34846582,35.4830^{\circ} \mathrm{W}, 34.3499^{\circ} \mathrm{N}$,

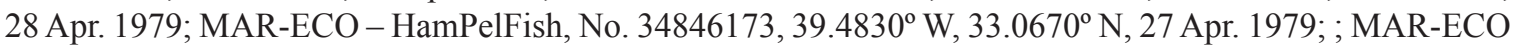

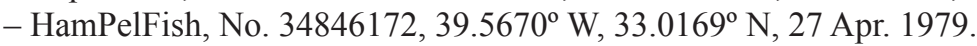

Diaphus luetkeni (Brauer, 1904) - No common name; (2) (3)

The presence of the species D. luetkeni is reported off the Azores, in the PECS area (160 NM southwest of the Georgiy Zima Seamount), SOC, Discovery No. 740614_8_FIS_214005, 20.1666 ${ }^{\circ}$ W, 39.9741 ${ }^{\circ}$ N, 3 Oct. 1970.

Diaphus metopoclampus (Cocco, 1829) - Spothead lantern fish; (1), Divertido, Escolarinho or Lobisomem (2), Romeirinho (3)

Diaphus mollis Tåning, 1928 - Lanternfish; Divertido, Escolarinho or Lobisomem(2), Romeirinho (3) Diaphus perspicillatus (Ogilby, 1898) - Transparent lantern fish; (2)

There are records of the species D. perspicillatus off the Azores, in the PECS area (56 NM southwest of the Hayes Fracture Zone, 40 NM SSW of the Konstatinov Ridge and 41 NM NNE of the Albany Seamount), SOC, Discovery No. 1022825 _85_FIS_ $214015,31.5567^{\circ}$ W, $32.9758^{\circ}$ N, 3 Nov. 1980; SOC, Discovery No. 1022826_86_FIS_214015, 31.0141 ${ }^{\circ} \mathrm{W}, 33.0141^{\circ} \mathrm{N}, 3$ Nov. 1980; MAR-ECO - HamPelFish, No. 34847868,

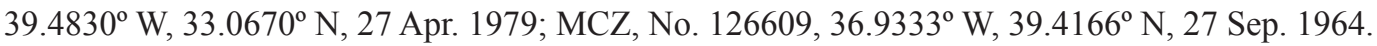

* Diaphus rafinesquii (Cocco, 1838) - White-spoted lantern fish; Ferreiro (1), Divertido, Escolarinho or Lobisomem (2), Romeirinho (3)

Diaphus splendidus (Brauer, 1904) - Horned lanternfish; (2)

The occurrence of this species is reported off the Azores, in the PECS area (63 NM southwest of the Hayes Fracture Zone), MAR-ECO - HamPelFish, No. 34844386, 39.5670 W, 33.0169º N, 27 Apr. 1979.

Diaphus subtilis Nafpaktitis, 1968 - No common name; (2), Romeirinho(3)

There are records of the species D. subtilis off the Azores, in the PECS area (30 NM SSW of the Konstatinov Ridge and 110 NM south of the Marsala Seamount), SOC, Discovery No. 1023202_85_FIS_214010,31.6016 W, $33.0525^{\circ} \mathrm{N}, 13$ Nov. 1980; SOC, Discovery No. 1023204_84_FIS_214010, $31.5625^{\circ} \mathrm{W}, 32.9842^{\circ} \mathrm{N}, 13$

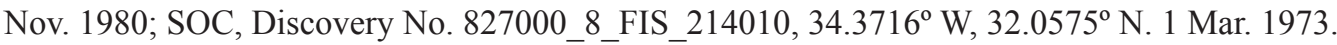

Diaphus termophilus Tåning, 1928 - Taaning's lantern fish; (3) 
Diogenichthys atlanticus (Tåning, 1928) - Lanternfish; (1), Divertido, Escolarinho or Lobisomem (2), Romeirinho (3)

$\square \quad$ Electrona risso (Cocco, 1829) - Electric lantern fish; (1), Divertido, Escolarinho or Lobisomem (2), Romeirinho (3)

$\square$ Gonichthys cocco (Cocco, 1829) - Lanternfish; (1), Divertido, Escolarinho or Lobisomem(2), Romeirinho (3)

․ Hygophum benoiti (Cocco, 1838)-Benoit's lantern fish; (1), Divertido, Escolarinho or Lobisomem (2), Romeirinho (3)

- Hygophum hygomii (Lütken, 1892) - Bermuda lantern fish; (1), Divertido, Escolarinho or Lobisomem (2), Romeirinho (3)

Hygophum macrochir (Günther, 1864) - No common name; (2) (3)

Hygophum reinhardtii (Lütken, 1892) - Lanternfish; (1), Divertido, Escolarinho or Lobisomem (2), Romeirinho (3)

Hygophum taaningi Becker, 1965 - Lanternfish; Divertido, Escolarinho or Lobisomem(2), Romeirinho (3)

Lampadena anomala Parr, 1928 - Lanternfish; Divertido, Escolarinho or Lobisomem(2), (3)

Lampadena chavesi Collett, 1905 - Lanternfish; Pirilampo(1), Divertido, Escolarinho or Lobisomem (2), Romeirinho (3)

Lampadena luminosa (Garman, 1899) - Luminous lanternfish; Pirilampo, (3)

Lampadena speculigera Goode \& Bean, 1896 - Lanternfish; (1), Divertido, Escolarinho or Lobisomem (2), Romeirinho (3)

- Lampadena urophaos atlantica Maul, 1969 - No common name; (2), Romeirinho (3)

Lampanyctus alatus Goode \& Bean, 1896 - No common name; (1) (2), Romeirinho (3)

There are records of this species to the south and in the west of the Azores, in the PECS area (Cruiser Tablemount, 40 NM southwest of the Konstantinov Ridge and 70 NM southwest of the Albany Seamount), SOC, Discovery No. 826500_8_FIS_461003, 27.1967 W, 32.0650 N, 27 Feb. 1973; SOC, Discovery No. 102320484 FIS $461003,31.5625^{\circ}$ W, $32.9842^{\circ}$ N, 13 Nov. 1980; SOC, Discovery No. 102282585 FIS $461003,31.5567^{\circ} \mathrm{W}, 32.9758^{\circ} \mathrm{N}, 3$ Nov. $1980 ; \mathrm{MCZ}$, No. $113874,-37.6000^{\circ} \mathrm{W}, 37.4833^{\circ} \mathrm{N}, 2$ Jul. 1969.

* Lampanyctus crocodilus (Risso, 1810) - Jewel lanternfish; (1), Divertido, Escolarinho or Lobisomem (2), Romeirinho (3)

Lampanyctus festivus Tåning, 1928 - Lanternfish; (1), Divertido, Escolarinho or Lobisomem(2), Romeirinho (3)

Lampanyctus intricarius Tåning, 1928 - Lanternfish; (1), Divertido, Escolarinho or Lobisomem(2), Romeirinho (3)

Lampanyctus macdonaldi (Goode \& Bean, 1896) - No common name; (1) (2)

Lampanyctus photonotus Parr, 1928 - Lanternfish; (1), Divertido, Escolarinho or Lobisomem(2), Romeirinho (3)

$\square \quad$ Lampanyctus pusillus (Johnson, 1890) - Lanternfish; (1), Divertido, Escolarinho or Lobisomem(2), Romeirinho (3)

Lepidophanes gaussi (Brauer, 1906) - Lanternfish; (1), Divertido, Escolarinho or Lobisomem(2), Romeirinho (3)

Lepidophanes guentheri (Goode \& Bean, 1896) - Lanternfish; Divertido, Escolarinho or Lobisomem (2), Romeirinho (3)

Lobianchia dofleini (Zugmayer, 1911) - Dofleini's lantern fish; (1), Divertido, Escolarinho or Lobisomem (2), Romeirinho (3)

Lobianchia gemellarii (Cocco, 1838) - Lanternfish; (1), Divertido, Escolarinho or Lobisomem(2), Romeirinho (3)

- Loweina interrupta (Tåning, 1928) - Lanternfish; (1), Divertido, Escolarinho or Lobisomem(2) Loweina rara (Lütken, 1892) - Lanternfish; Divertido, Escolarinho or Lobisomem (2), Romeirinho (3) Myctophum affine (Lütken, 1892) - Metallic lanternfish; (2) 
This species is reported off the Azores, in the PECS area (65 NM northeast of the Marsala Seamount), Station $53,33.0166^{\circ} \mathrm{W}, 34.9833^{\circ} \mathrm{N}$ (Murray \& Hjort 1912).

Myctophum nitidulum Garman, 1899 - Spotted lanterfish; Divertido, Escolarinho or Lobisomem (2), Romeirinho (3)

* Myctophum punctatum Rafinesque, 1810 - Spotted lanternfish; (1), Divertido, Escolarinho or Lobisomem (2), Romeirinho (3)

Myctophum selenops Tåning, 1928 - Wisner’s lantern fish; (2), Romeirinho(3)

There are records of M. selenops off the Azores, in the PECS area (110 NM northwest of the Atlantis Seamount, 45 NM SSW of the Oceanographer Fracture Zone and 54 NM southwest of the Hayes Fracture Zone), MARECO - HamPelFish, No. 34849353, 31.8829 W, $35.4000^{\circ}$ N, 29 Apr. 1979; MAR-ECO - HamPelFish, No. $34849350,35.4830^{\circ} \mathrm{W}, 34.4830^{\circ} \mathrm{N}, 28$ Apr. 1979; MAR-ECO - HamPelFish, No. 34849351, $35.4000^{\circ} \mathrm{W}$, $34.3330^{\circ}$ N, 28 Apr. 1979; MAR-ECO - HamPelFish, No. 34849352, 35.3670 W, $34.3499^{\circ}$ N, 28 Apr. 1979; MNHN, No. 1980-1346, $35.4000^{\circ}$ W, $34.3330^{\circ}$ N, Apr. 1979; MAR-ECO - HamPelFish, No. 34849349 , $39.4830^{\circ} \mathrm{W}, 33.0670^{\circ} \mathrm{N}, 27$ Apr. 1979.

Nannobrachium atrum (Tåning, 1928) - Lanternfish; (1), Divertido, Escolarinho or Lobisomem (2), Romeirinho (3)

Nannobrachium cuprarium (Tåning, 1928) - No common name; (1) (2), Romeirinho (3)

Nannobrachium lineatum (Tåning, 1928) - No common name; (1) (2), Romeirinho (3)

Nannobrachium nigrum Günther, 1887 - No common name.

The species $N$. nigrum is regarded as being present off Mainland Portugal (occurrence reported at GBIF: ID 350188791 ). The specimen was caught at $37^{\circ} 32^{\prime} 31.20^{\prime \prime} \mathrm{N}, 12^{\circ} 57^{\prime} 3.60^{\prime \prime} \mathrm{W}$ and is deposited at Yale University Peabody Museum (catalogue number YPM ICH 004979). According to Zahuranec (2000), N. nigrum is found in the tropical Pacific, excluding the Eastern Tropical Pacific Region, throughout the East Indian archipelago as far west as the extreme eastern tropical Indian Ocean. For this reason, the occurrence of N. nigrum is very doubtful and the species was not accounted for in the total number of species in the current list.

Notolychnus valdiviae (Brauer, 1904) - No common name; (1), Divertido, Escolarinho or Lobisomem (2), Romeirinho(3)

$\square \quad$ Notoscopelus bolini Nafpaktitis, 1975 - Lanternfish; (1), Divertido, Escolarinho or Lobisomem (2) Notoscopelus caudispinosus (Johnson, 1863) - Lobisomem; Divertido, Escolarinho or Lobisomem (2), Romeirinho (3)

$\square \quad$ Notoscopelus elongatus (Costa, 1844) - No common name; (1)

The subspecies Notoscopelus elongatus kroyeri (Malm, 1861), reported from the Azores and Madeira, should probably be considered as Notoscopelus kroyeri (Malm, 1861). The specimen collected in 1969, at the south coast of Portugal, MNHN 1978-0444, $-8.8500^{\circ} \mathrm{W}, 36.4329^{\circ} \mathrm{N}$, was identified as $N$. elongatus.

Notoscopelus kroyeri (Malm, 1861) - Lanternfish; (1), Divertido, Escolarinho or Lobisomem(2), (3) The subspecies Notoscopelus elongatus kroyeri (Malm, 1861) is synonymous with the species $N$. kroyeri, it is reported off Madeira, in the PECS area (Josephine Bank), MCZ, No. 104269, -13.3166 W, 36.666 N, 14 Jul. 1972.

$\square \quad$ Notoscopelus resplendens (Richardson, 1845) - Patchwork lampfish; (1), Divertido, Escolarinho or Lobisomem (2), Romeirinho (3)

Protomyctophum arcticum (Lütken, 1892) - Lanternfish; (1), Divertido, Escolarinho or Lobisomem (2) Scopelopsis multipunctatus Brauer, 1906 - No common name; (3)

Symbolophorus rufinus (Tåning, 1928) - No common name; (2)

S. rufinus is reported from the Azores, in the PECS area (Plato Seamount, 38 NM SSW and 100 NM south of the Konstatinov Ridge), SOC, Discovery No. 1022209_86_FIS_785001, 30.0841 ${ }^{\circ}$ W, 33.1292 ${ }^{\circ}$ N, 1980.10.30; SOC, Discovery No. 1022217_85_FIS_785001, $30.0625^{\circ} \mathrm{W}, 33.0633^{\circ} \mathrm{N}, 31$ Oct. 1980 ; SOC, Discovery No. 1022216_84_FIS_785001, 30.0358 ${ }^{\circ}$ W, $33.0900^{\circ}$ N, 31 Oct. 1980; SOC, Discovery No. 1022824_84_FIS_785001, $31.5233^{\circ}$ W, $32.9400^{\circ}$ N, 3 Nov. 1980; SOC, Discovery No. 1022825_85 FIS_785001, $31.5567^{\circ} \mathrm{W}, 32.9758^{\circ} \mathrm{N}, 3$ Nov. 1980 ; MCZ, No. $103498,30.6833^{\circ} \mathrm{W}, 31.7333^{\circ} \mathrm{N}, 6$ Sep. 1973 ;

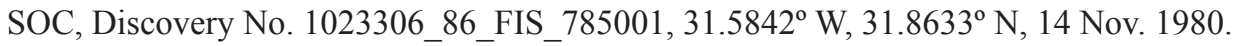

Symbolophorus veranyi (Moreau, 1888) - Large-scale lantern fish; (1), Divertido, Escolarinho or Lobisomem (2), Romeirinho(3) 
Taaningichthys bathyphilus (Tåning, 1928) - No common name; (2), Romeirinho (3)

Taaningichthys minimus (Tåning, 1928) - No common name; Escolarinho (2), Romeirinho (3)

$\square \quad$ Taaningichthys paurolychnus Davy, 1972 - No common name; (2), Romeirinho (3)

Order Lampriformes

Family Lampridae

* Lampris guttatus (Brünnich, 1788) - Opah; Peixe-cravo (1) (2) (3), Joaninha (2)

Family Stylephoridae

Stylephorus chordatus Shaw, 1791 - Tube-eye; (2)

Family Lophotidae

* Lophotus lacepede Giorna, 1809 - Crested oarfish; (1) (3)

Family Radiicephalidae

Radiicephalus elongatus Osório, 1917 - Tapertail; (2) (3)

Family Trachipteridae

Trachipterus arcticus (Brünnich, 1788) - Dealfish; Rei-dos-arrenques (1), (2) (3)

* Trachipterus trachypterus (Gmelin, 1789) - Mediterranean dealfish; Peixe-tábua (1), (2) (3)

$\diamond \quad$ Zu cristatus (Bonelli, 1819) - Scalloped ribbonfish; (2) (3)

Family Regalecidae

* Regalecus glesne Ascanius, 1772 - King of herrings; Relangueiro (1), Peixe-real, Regaleco or Reidos-arenques (2), (3)

Order Polymixiiformes

Family Polymixiidae

$\square \quad$ Polymixia nobilis Lowe, 1838 - Stout beardfish; Salmonete-do-alto(2) (3)

Order Gadiformes

Family Macrouridae

Asthenomacrurus victoris Sazonov \& Shcherbachev, 1982 - No common name; (2)

Bathygadus favosus Goode \& Bean, 1886 - No common name; (1) (2), Lagartixa-do-mar(3)

This species is recorded off the Azores, in the PECS area (in the Mid-Atlantic Ridge: 170 NM west of the

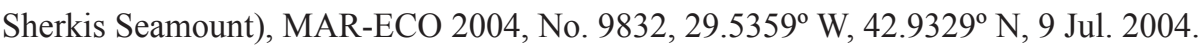

Bathygadus melanobranchus Vaillant, 1888 - Vaillant's grenadier; (1), Peixe-rato or Rato(2), (3)

This species is often confused with B. favosus. According to Iwamoto (2003), B. melanobranchus is most similar to B. favosus in its external features, but differs in counts of pyloric caeca (9-12 compared with 16-27 in B. favosus).

Cetonurus globiceps (Vaillant, 1884) - Globehead grenadier; (1), Peixe-rato or Rato(2), Peixelagartixa (3)

* Caelorinchus caelorincus (Risso, 1810) - Hollowsnout grenadier; Lagartixa-do-mar(1) (3); Ratobicudo (2), Lagartixa (3) 
Caelorinchus labiatus (Köelher, 1896) - Spearsnouted grenadier; (1), Peixe-rato or Rato(2)

Caelorinchus occa (Goode \& Bean, 1885) - Spear-snouted grenadier; (1), Peixe-rato or rato(2)

Coryphaenoides armatus (Hector, 1875) - Abyssal grenadier; (1), Peixe-rato or Rato (2), Lagartixade-natura (3)

Coryphaenoides asper Günther, 1877 - No common name;

The species $C$. asper is regarded as being present off the Azores (GBIF occurrences). Two specimens are deposited in the fish collections of the Muséum National d'Histoire Naturelle, MNHN No. 2009-0420, $25.4680^{\circ} \mathrm{W}, 39.4180^{\circ} \mathrm{N}, 17$ Oct. 1971 ; MNHN, No. $2009-0419,25.4680^{\circ} \mathrm{W}, 39.4180^{\circ} \mathrm{N}, 17$ Oct. 1971. In 2009 the specimens were re-evaluated and identified as Coryphaenoides sp. The occurrences from the Azores are therefore considered as doubtful and the presence of this species off the Azores should not be accepted. For this reason this species is not counted in the present list.

Coryphaenoides brevibarbis (Goode \& Bean, 1896) - Shortbeard brenadier; (2)

Coryphaenoides carapinus Goode \& Bean, 1883 - Carapine grenadier; (1), Peixe-rato or Rato (2), (3) Coryphaenoides guentheri (Vaillant, 1888) - Günther's grenadier; (1), Peixe-rato or Rato (2)

Coryphaenoides leptolepis Günther, 1877 - No common name; (1), Peixe-rato or Rato(2)

Coryphaenoides mediterraneus (Giglioli, 1893) - Mediterranean grenadier; (1), Peixe-rato or Rato (2) Coryphaenoides profundicolus (Nybelin, 1957) - Deepwater grenadier; (1) (3)

$\square \quad$ Coryphaenoides rudis Günther, 1878 - Madeira brenadier; (2) (3)

Coryphaenoides rupestris Gunnerus, 1765 - Roundnose grenadier; Lagartixa-da-rocha, (2) (3)

The occurrence of the species C. rupestris is reported off Madeira, in the PECS area (Josephine Bank), BMNH, No. 1995.7.25.17, $14.3002^{\circ} \mathrm{W}, 36.7336^{\circ} \mathrm{N}$; 11 Apr. 1972; SOC, Discovery No. 785705_210_FIS_119003, $14.5199^{\circ} \mathrm{W}, 36.7824^{\circ} \mathrm{N}, 12$ Apr. 1972.

$\square \quad$ Coryphaenoides thelestomus Maul, 1951 - Roughlip grenadier; (3)

Coryphaenoides zaniophorus (Vaillant, 1888) - Thickbeard grenadier; (1), Peixe-lagartixa (3)

Echinomacrurus mollis Roule, 1916 - No common name; (3)

Gadomus arcuatus (Goode \& Bean, 1886) - Doublethread grenadier; (1) (2)

* Gadomus dispar (Vaillant, 1888) - No common name; (1) (2)

* Gadomus longifilis (Goode \& Bean, 1885) - Treadfin grenadier; (1), Peixe-rato or Rato (2), (3)

$\square \quad$ Hymenocephalus gracilis Gilbert \& Hubbs, 1920 - Graceful grenadier; (2)

There are records of $\mathrm{H}$. gracilis off the Azores, in the PECS area (Great Meteor Tablemount), SDSC, No. $22989,28.6399^{\circ} \mathrm{W}, 30.0849^{\circ} \mathrm{N}, 26$ Jul. 1967 ; SDSC, No. $16268,28.6000^{\circ} \mathrm{W}, 30.0333^{\circ} \mathrm{N}, 1$ Jul. 1982; SDSC, No. $9118,28.6066^{\circ} \mathrm{W}, 29.9633^{\circ} \mathrm{N}, 26$ Feb. 1970 ; SDSC, No. $21253,28.3316^{\circ} \mathrm{W}, 29.9358^{\circ} \mathrm{N}, 10 \mathrm{Sep} .1998$; SDSC, No. 9116, $28.3850^{\circ} \mathrm{W}, 29.9149^{\circ} \mathrm{N}, 8$ Sep. 1998; SDSC, No. 22988, $28.3966^{\circ} \mathrm{W}, 29.8366^{\circ} \mathrm{N}, 22 \mathrm{Jul}$. 1967; SDSC, No. 9117, 28.3816 W, 29.7699 N, 8 Sep. 1998.

* Hymenocephalus italicus Giglioli, 1884 - Glasshead grenadier; Lagartixa-prateada(1), Peixe-rato or Rato(2), (3)

* Malacocephalus laevis (Lowe, 1843) - Softhead grenadier; Peixe-rato(1) (2), Rato-redondo(2), Lagartixa-do-mar (3)

- Nezumia aequalis (Günther, 1878) - Common Atlantic grenadier; Lagartixa-do-mar(1), Ratoredondo(2), Lagartixa (3)

Nezumia bairdii (Goode \& Bean, 1877) - Marlin-spike grenadier; (2) (3)

Nezumia longebarbata (Roule \& Angel, 1933) - Bluntnose grenadier; Lagartixa-do-mar(3)

Nezumia sclerorhynchus (Valenciennes, 1838) - Roughtip grenadier; Lagartixa-áspera (1), Peixerato or Rato(2), (3)

Odontomacrurus murrayi Norman, 1939 - Roundhead grenadier; Peixe-rato or Rato (2), Cobaia (3)

Paracetonurus flagellicauda (Koefoed, 1927) - No common name; (2)

$\square \quad$ Sphagemacrurus grenadae (Parr, 1946) - Pugnose grenadier; (1) (2)

The presence of S. grenadae is reported from the Azores, in the PECS area, Plateau Seamount (Shcherbachev et al. 1985) and Hyeres Seamount (Kukuev 2002), $29.0000^{\circ} \mathrm{W}, 31.0000^{\circ} \mathrm{N}$.

Sphagemacrurus hirundo (Collett, 1896) - Swallow grenadier; Peixe-rato or Rato(2), (3)

Squalogadus modificatus Gilbert \& Hubbs, 1916 - No common name; (2) 
Trachonurus sulcatus (Goode \& Bean, 1885) - Bristly grenadier; (1), Lagartixa-negra(2)

Trachonurus villosus (Günther, 1877) - Bristly grenadier; (1), Peixe-rato or Rato (2), (3)

* Trachyrincus scabrus (Rafinesque, 1810) - Roughsnout grenadier; Peixe-lima (1)

\section{Family Moridae}

Antimora rostrata (Günther, 1878) - Blue antimora; Mora-azul(1), (2)

Eretmophorus kleinenbergi Giglioli, 1889 - No common name; (2)

E. kleinenbergi is reported off the Azores, in the PECS area (Maxwell Fracture Zone), ISH, No. 583-1982, $27.1500^{\circ} \mathrm{W}, 47.8800^{\circ} \mathrm{N}$.

* $\square$ Gadella maraldi (Risso, 1810) - Gadella; Abrótea-de-natura (1) (3), Gadela(2)

Guttigadus latifrons (Holt \& Byrne, 1908) - No common name; (2)

* Halargyreus johnsonii Günther, 1862 - Slender codling; (1) (2), Abrótea-de-natura (3)

Laemonema robustum Johnson, 1862 - Robust mora; Abrótea or Abrótea-de-natura (3)

- Laemonema yarrellii (Lowe, 1838) - No common name; (2), Abrótea-de-natura (3)

The occurrence of $L$. yarrellii is reported from the Azores, in the PECS area (sixteen records in the Great Meteor Tablemount), USNM, No. 304412, $28.5000^{\circ} \mathrm{W}, 29.8330^{\circ} \mathrm{N}, 1970.02 .26$; SDSC, No. 22998, $28.4966^{\circ}$ W, $29.8366^{\circ}$ N, 18 Jul. 1967; SDSC, No. 22999, $28.6667^{\circ}$ W, $30.0499^{\circ}$ N, 25 Jul. 1967; SDSC, No. 16289, $28.2566^{\circ} \mathrm{W}, 29.9733^{\circ} \mathrm{N}, 26$ Jun. 1982.

$\diamond \quad$ Lepidion eques (Günther, 1887) - North Atlantic codling; (2)

Bañón et al. (2012) discovered that the COI DNA sequencing along with morphological and meristic analyses strongly suggest that there are no specific differences between the Atlantic Lepidion eques and the Mediterranean species L. lepidion (Risso, 1810). Therefore, they proposed $L$. eques as a junior synonym of $L$. lepidion.

Lepidion guentheri (Giglioli, 1880) - Morid cod; (1), Juliana-pequena (2), (3) Lepidion schmidti Svetovidov, 1936 - Schmidt's cod; (2)

* $\square$ Mora moro (Risso, 1810)-Common mora; Mora (1), Escamuda-branca, Melg or Juliana (2), Abróteado-alto, Robaldo-branco or Buzia (3)

Physiculus dalwigki Kaup, 1858 - Black codling; Abrótea-de-natura (1), (2) (3)

Rhynchogadus hepaticus (Facciolà, 1884) - No common name; (2)

Family Melanonidae

$\diamond \quad$ Melanonus zugmayeri Norman, 1930 - Arrowtail; (1) (2) (3)

Family Merlucciidae

ㅁyconus brachycolus Holt \& Byrne, 1906 - No common name; (1) (2) (3)

- Macruronus maderensis Maul, 1951 - No common name; (3)

* $\square$ Merluccius merluccius (Linnaeus, 1758) - European hake; Pescada-branca (1), (2) (3)

Family Phycidae

* Phycis blennoides (Brünnich, 1768) - Greater forkbeard; Abrótea-do-alto (1) (2) (3), Abrótea (1), Juliana or Melga (2)

* $\square$ Phycis phycis (Linnaeus, 1766) - Forkbeard; Abrótea-da-costa (1), Abrótea(2) (3)

Family Gadidae

* Ciliata mustela (Linnaeus, 1758) - Fivebearded rockling; Laibeque-de-cinco-barbilhos (1)

* Gadiculus argenteus argenteus Guichenot, 1850 - Silvery pout; Badejinho (1), (2) 
* Gaidropsarus biscayensis (Collett, 1890) - Mediterranean bigeye rockling; Laibeque-olhogrande (1), (3)

Gaidropsarus granti (Regan, 1903) - Grant’s rockling; Viúva-do-alto or Aranha-do-alto (2), (3)

$\square \quad$ Gaidropsarus guttatus (Collet, 1890) - No common name; Aranha-da-pedra or Viúva (2), Abróteade-poça (3)

$\square \quad$ Gaidropsarus macrophthalmus (Günther, 1867) - Bigeye rockling; (1)

* Gaidropsarus mediterraneus (Linnaeus, 1758) - Shore rockling; Laibeque (1)

* Gaidropsarus vulgaris (Cloquet, 1824) - Three-bearded rockling; Laibeque-de-três-barbilhos (1), (3)

* Merlangius merlangus (Linnaeus, 1758) - Whiting; Badejo (1)

* $\diamond$ Micromesistius poutassou (Risso, 1827) - Blue whiting; Verdinho (1) (2), Pichelim(2)

Molva dypterygia (Pennant, 1784) - Blue ling; Donzela-azul(1), (2)

This species is regarded as being present north of the Azores (Hareide \& Garnes 2001), in the PECS area (Mid-Atlantic Ridge, $43^{\circ}-44^{\circ} \mathrm{N}$ ).

* Molva macrophthalma (Rafinesque, 1810) - Spanish ling; Donzela-do-Mediterrâneo (1), Pescadados-Açores(2)

* Molva molva (Linnaeus, 1758) - Ling; Maruca (1)

* Pollachius pollachius (Linnaeus, 1758) - Pollack; Juliana (1)

* Raniceps raninus (Linnaeus, 1758) - Tadpole fish; Rainúnculo-negro(1)

* Trisopterus luscus (Linnaeus, 1758) - Bib; Faneca (1)

* Trisopterus minutus (Linnaeus, 1758) - Poor cod; Fanecão(1)

\section{Order Ophidiiformes \\ Family Carapidae}

Carapus acus (Brünnich, 1768) - Pearl fish; (1), Peixe-cobrelo(2) (3)

$\square \quad$ Echiodon dentatus (Cuvier, 1829) - No common name; (2) (3)

E. dentatus is reported off Madeira (Josephine Bank) and to the south of the Azores (Great Meteor Tablemount), but in all those records the specimens were captured outside their EEZ's, in the PECS area, SDSC, No. 23048, $14.2533^{\circ} \mathrm{W}, 36.7050^{\circ} \mathrm{N}, 2$ Jul. 1967; SDSC, No. 23049, $28.4966^{\circ} \mathrm{W}, 29.8366^{\circ} \mathrm{N}, 18$ Jul. 1967; SDSC, No. $23050,28.3966^{\circ} \mathrm{W}, 29.8366^{\circ} \mathrm{N}$; 22 Jul. 1967; SDSC, No. 23051, $28.5250^{\circ} \mathrm{W}, 30.0500^{\circ} \mathrm{N}, 25$ Jul. 1967; SDSC, No. $9310,28.3850^{\circ} \mathrm{W}, 29.9149^{\circ} \mathrm{N}, 8$ Sep. 1998.

$\diamond \quad$ Echiodon drummondii Thompson, 1837 - No common name; (2)

Family Ophidiidae

Acanthonus armatus Günther, 1878 - No common name; (2)

Barathrites iris Zugmayer, 1911 - No common name; (2)

Bassozetus compressus (Günther, 1878) - No common name; (1) (3)

Bassozetus levistomatus Machida, 1989 - No common name; (3)

B. levistomatus is reported off Madeira, in the PECS area (233 NM WSW of Madeira in the Madeira Plain),

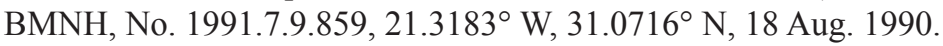

Bassozetus taenia (Günther, 1887) - No common name; (3)

B. laticeps is reported off Madeira, in the PECS area (220 NM WSW of Madeira in the Madeira Plain),

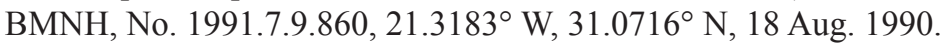

Bathyonus laticeps (Günther, 1878) - No common name; (2) (3)

B. laticeps is reported off the Azores, in the PECS area (85 NM northeast of the Marsala Seamount) (Koefoed 1932), $33.0166^{\circ} \mathrm{W}, 34.9833^{\circ} \mathrm{N}$.

$\diamond \quad$ Benthocometes robustus (Goode \& Bean, 1886) - No common name; (1), Abadejo-cometa(2)

Brotulotaenia brevicauda Cohen, 1974 - No common name; (2)

Brotulotaenia crassa Parr, 1934 - No common name; (1) (2) (3)

Brotulotaenia nigra Parr, 1933 - No common name; (2) (3) 
This species is reported from the Azores, in the PECS area (94 NM southwest of the Konstantinov Ridge), SOC, Discovery No. 1023328_86_FIS_078001, 31.5900 W, 32.0316 ${ }^{\circ}$ N, 16 Nov. 1980.

Holcomycteronus squamosus (Roule, 1916) - No common name; (2) (3)

Lamprogrammus niger Alcock, 1891 - No common name; (2)

Monomitopus metriostoma (Vaillant, 1888) - No common name; (1) (2)

* Ophidion barbatum Linnaeus, 1758 - Snake blenny; Peixe-cobrelo(1), (3)

$\diamond \square$ Parophidion vassali (Risso, 1810) - No common name; (2) (3)

Penopus microphthalmus (Vaillant, 1888) - No common name; (2)

Porogadus miles Goode \& Bean, 1885 - Slender cuskeel; (2)

Spectrunculus crassus (Vaillant, 1888) - No common name; (2)

Spectrunculus grandis (Günther, 1877) - Pudgy cuskeel; (1) (2) (3)

Family Bythitidae

$\square \quad$ Bellottia apoda Giglioli, 1883 - No common name; (1) (2) (3)

This species occurs off the Azores, in the PECS area (Great Meteor Tablemount), SDSC, No. 23047, 28.4966 ${ }^{\circ}$ W, $29.8366^{\circ} \mathrm{N}, 18 \mathrm{Jul} .1967$.

Cataetyx alleni (Byrne, 1906) - No common name; (1)

$\square \quad$ Cataetyx laticeps Koefoed, 1927 - No common name; (2)

Grammonus ater (Risso, 1810) - No common name; (2)

Family Aphyonidae

Aphyonus gelatinosus Günther, 1878 - No common name; (2) Barathronus multidens Nielsen, 1984 - No common name; (1) Barathronus parfaiti (Vaillant, 1888) - No common name; (2) Meteoria erythrops Nielsen, 1969 - No common name; (2) Nybelinella erikssoni (Nybelin, 1957) - No common name; (1)

The species $N$. erikssoni is regarded as being present off the Portuguese mainland EEZ, based on its geographical distribution (Van der Land et al. 2001).

Sciadonus galatheae (Nielsen, 1969) - No common name; (2)

The species S. galatheae is regarded as being present off the coast of Azores (Rannou et al. 1974; Nielsen \& Møller 2008), MNHN, No. 1973-0033.

Sciadonus pedicellaris Garman, 1899 - No common name; (2)

Family Parabrotulidae

Leucobrotula adipata Koefoed, 1952 - No common name; (1) (2)

Parabrotula plagiophthalma Zugmayer, 1911 - No common name; (2) (3)

Order Batrachoidiformes

Family Batrachoididae

* Halobatrachus didactylus (Bloch \& Schneider, 1801) - Lusitanian toadfish; Charroco or Xarroco (1), (3)

Order Lophiiformes

Family Lophiidae

* Lophius budegassa Spinola, 1807 - Blackbellied angler; Tamboril-sovaco-preto (1), (2)

* Lophius piscatorius Linnaeus, 1758 - Angler; Tamboril(1) (2), Peixe-diabo or Peixe-engana(2) 
Family Antennariidae

Antennarius multiocellatus (Valenciennes, 1837) - Longlure frogfish; (2)

$\square \quad$ Antennarius nummifer (Cuvier, 1817) - Spotfin frogfish; (2) (3)

․ Fowlerichthys radiosus Garman, 1896 - Singlespot frogfish; (2) (3)

$\diamond \quad$ Fowlerichthys senegalensis Cadenat, 1959 - Senegalese frogfish; (2)

Histrio histrio (Linnaeus, 1758) - Sargassumfish; (1) (2) (3)

Family Chaunacidae

Chaunacops coloratus (Garman, 1899) - No common name; (2)

This species occurs off the Azores, in the PECS area (68 NM west of the Oceanographer Fracture Zone), MNHN, No. 1996-0225, 36.3670 W, 34.8170 N, 2 Sep. 1995.

Chaunacops roseus (Barbour, 1941) - No common name; (2)

$* \diamond \square$ Chaunax pictus Lowe, 1846 - No common name; Sapo-mole (1), (2) (3)

Chaunax suttkusi Caruso, 1989 - No common name; (2)

Family Caulophrynidae

Caulophryne jordani Goode \& Bean, 1896 - No common name; (2)

The species C. jordani is regarded as being present to the southwest of Madeira (Bertelsen, E. in Whitehead et al. 1986), but was caught outside the EEZ and outside the proposed extension of the Portuguese continental shelf.

Caulophryne polynema Regan, 1930 - No common name; (2) (3)

Family Neoceratiidae

Neoceratias spinifer Pappenheim, 1914 - No common name; (2)

There is a record of this species from off the Azores, in the PECS area (81 NM northeast of the Marsala Seamount), $33.0166^{\circ} \mathrm{W}, 34.9833^{\circ} \mathrm{N}$ (Koefoed 1932).

Family Melanocetidae

Melanocetus johnsonii Günther, 1864 - Humpback anglerfish; (1) (2) (3)

Melanocetus murrayi Günther, 1887 - No common name; (2) (3)

Family Himantolophidae

$\square \quad$ Himantolophus albinares Maul, 1961 - No common name; (2), Farol(3)

H. albinares occurs off the Azores, in the PECS area (45 NM southwest of the Oceanographer Fracture Zone),

MAR-ECO - HamPelFish, No. 34850546, 35.4830 W, 34.3499 N, 28 Apr. 1979.

Himantolophus brevirostris (Regan, 1925) - No common name; (2)

Himantolophus compressus (Osório, 1912) - No common name; (1) (3)

* Himantolophus groenlandicus Reinhardt, 1837 - No common name; Peixe-de-farol(1), (2) (3)

$\square \quad$ Himantolophus mauli Bertelsen \& Krefft, 1988 - No common name; (3)

Family Diceratiidae

Bufoceratias wedli (Pietschmann, 1926) - No common name; (1) (3)

Family Oneirodidae

Chaenophryne draco Beebe, 1932 - Smooth dreamer; (1) (2) (3) 
Chaenophryne longiceps Regan, 1925 - No common name; (2) (3)

Chaenophryne ramifera Regan \& Trewavas, 1932 - No common name; (2)

Ctenochirichthys longimanus Regan \& Trewavas, 1932 - No common name; (3)

Dolopichthys allector Garman, 1899 - No common name; (2) (3)

A single specimen was observed to the northeast of the Azores, in the PECS area (64 NM NNW of the Kings Trough), MAR-ECO - HamPelFish, No. 34848312, 22.2670 W, 44.9000 N, 3 May 1979.

Dolopichthys danae Regan, 1926 - No common name; (3)

Dolopichthys jubatus Regan \& Trewavas, 1932 - No common name; (2) (3)

Dolopichthys karsteni Leipertz \& Pietsch, 1987 - No common name; (1) (2) (3)

This species occurs off the Azores, in the PECS area (156 NM southwest of the Altair Seamount), MCZ, No. 149624, 35.5950 $\mathrm{W}, 42.0950^{\circ} \mathrm{N}, 3$ Jul. 1978.

Dolopichthys longicornis Parr, 1927 - No common name; (2)

Dolopichthys pullatus Regan \& Trewavas, 1932 - No common name; (2)

D. pullatus is recorded off the Azores, in the PECS area (25 NM WNW of the Sherkis Seamount and 108 NM SSW of the Olympus Knoll), MAR-ECO - HamPelFish, No. $34848316,26.2469^{\circ} \mathrm{W}, 43.2420^{\circ} \mathrm{N}$, 9 Jun. 1982; MAR-ECO - HamPelFish, No. 34848317, 28.4419º W, $43.6969^{\circ}$ N, 10 Jun. 1982.

Leptacanthichthys gracilispinis (Regan, 1925) - No common name; (2) (3)

Lophodolos acanthognathus Regan, 1925 - No common name; (1) (2)

Microlophichthys microlophus (Regan, 1925) - No common name; (2) (3)

Oneirodes anisacanthus (Regan, 1925) - No common name; (3)

Oneirodes clarkei Swinney \& Pietsch, 1988 - No common name; (3)

Oneirodes eschrichtii Lütken, 1871 - Bulbous dreamtail; (1) (2) (3)

Oneirodes macronema (Regan \& Trewavas, 1932) - No common name; (2)

Oneirodes macrosteus Pietsch, 1974 - No common name; (2)

Oneirodes myrionemus Pietsch, 1974 - No common name; (3)

Oneirodes posti Bertelsen \& Grobecker, 1980 - No common name; (2)

This species occurs off the Azores, in the PECS area (114 NM south of the Lucky Strike Seamount), MAR-

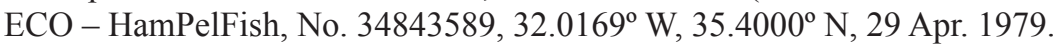

Pentherichthys venustus (Regan \& Trewavas, 1932) - No common name; (3)

Phyllorhinichthys micractis Pietsch, 1969 - No common name; (2)

Puck pinnata Pietsch, 1978 - No common name; (2)

This species occurs off the Azores, in the PECS area (107 NM northwest of the Atlantis Seamount), MAR-

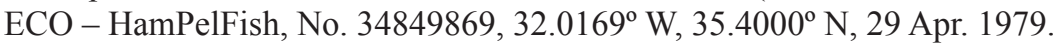

Spiniphryne gladisfenae (Beebe, 1932) - No common name; (2)

Family Thaumatichthyidae

Lasiognathus amphirhamphus Piestsch, 2005 - No common name; (2)

The species L. amphirhamphus (Holotype - BMNH 2003.11.16.12, $29.8450^{\circ} \mathrm{W}, 32.3700^{\circ} \mathrm{N}$ ) is regarded as being present on the Madeira Abyssal Plain (Pietsch 2005) off the southwest coast of Madeira Island, but the geographic coordinates of the occurrence is off the Azores, in the PECS area (54 NM southwest of the Plato Seamount).

Lasiognathus beebei Regan \& Trewavas, 1932 - No common name; (3)

- Lasiognathus saccostoma Regan, 1925 - No common name; (2) (3)

Family Centrophrynidae

Centrophryne spinulosa Regan \& Trewavas, 1932 - Horned lantern fish; (3)

Family Ceratiidae

Ceratias holboelli Krøyer, 1845 - Kroyer's deep sea anglerfish; (2) 
Cryptopsaras couesii Gill, 1883 - Triplewart seadevil; (2) (3)

Family Gigantactinidae

Gigantactis ios Bertelsen, Pietsch \& Lavenberg, 1981 - No common name; (3)

Gigantactis vanhoeffeni Brauer, 1902 - No common name; (2)

Family Linophrynidae

Haplophryne mollis (Brauer, 1902) - No common name; (2) (3)

Linophryne arcturi (Beebe, 1926) - No common name; (3)

Linophryne brevibarbata Beebe, 1932 - No common name; (2) (3)

Linophryne lucifer Collett, 1886 - No common name; (3)

Linophryne macrodon Regan, 1925 - No common name; (2)

$\square \quad$ Linophryne maderensis Maul, 1961 - No common name; (3)

$\square \quad$ Linophryne polypogon Regan, 1925 - No common name; (3)

Linophryne racemifera Regan \& Trewavas, 1932 - No common name; (3)

Linophryne sexfilis Bertelsen, 1973 - No common name; (3)

Photocorynus spiniceps Regan, 1925 - No common name; (3)

Order Mugiliformes

Family Mugilidae

$\square \quad$ Chelon labrosus (Risso, 1827) - Thicklip grey mullet; Tainha-liça(1), Tainha (1) (2) (3), Mugem(2) (3), Muja (2) (3), Muge (3)

* Liza aurata (Risso, 1810) - Golden grey mullet; Tainha-garrento(1)(2) (3), Tainha or Tainhaamarela (2) (3), Muge or Mugem(3)

* Liza ramada (Risso, 1827) - Thinlip grey mullet; Tainha-fataça(1), Tainha (2), Muge (3)

Wirtz et al. (2008) mention the need to find a specimen to confirm its presence at Madeira.

* Liza saliens (Risso, 1810) - Leaping mullet; Tainha-de-salto (1)

* Mugil cephalus Linnaeus, 1758 - Flathead grey mullet; Tainha-olhalvo(1), Tainha (3)

Wirtz et al. (2008) consider this a very doubtful record for Madeira.

Oedalechilus labeo (Cuvier, 1829) - Boxlip mullet; Tainha-sabão, (3)

Order Atheriniformes

Family Atherinidae

* Atherina boyeri Risso, 1810 - Big-scale sand smelt; Peixe-rei-do-Mediterrâneo (1), Peixe-rei or Piarda (1) (3)

The presence of $A$. boyeri off Madeira seems very doubtful (Wirtz et al. 2008).

$\square \quad$ Atherina hepsetus Linnaeus, 1758 - Mediterranean sand smelt; Peixe-rei-do-alto (1) (3), Guelro (3)

* Atherina presbyter Cuvier, 1829 - Sand smelt; Peixe-rei (1), (2), Guelro (3)

Order Beloniformes

Family Exocoetidae

Cheilopogon cyanopterus (Valenciennes, 1847) - Margined flyingfish; (1)

Cheilopogon exsiliens (Linnaeus, 1771) - Flying fish; (1), Peixe-voador (2), (3)

$\square \quad$ Cheilopogon furcatus (Mitchill, 1815) - Spotfin flyingfish; Peixe-voador or Voador(2) (3)

* Cheilopogon heterurus (Rafinesque, 1810) - Mediterranean flyingfish; Peixe-voador(1), (3) 
* Cheilopogon pinnatibarbatus pinnatibarbatus (Bennett, 1831) - Bennett's flyingfish; Peixevoador(1) (2), (3)

$\square \quad$ Exocoetus obtusirostris Günther, 1866 - Oceanic two-wing flyingfish; (1), Peixe-voador(2), (3)

$\square \quad$ Exocoetus volitans Linnaeus, 1758 - Tropical two-wing flyingfish; Peixe-voador, (2), Voador(3)

* Hirundichthys rondeletii (Valenciennes, 1847) - Black wing flyingfish; (1) (2) (3)

Hirundichthys speculiger (Valenciennes, 1847) - Mirrorwing flyingfish; (3)

Family Hemiramphidae

$\square \quad$ Hemiramphus balao Lesueur, 1821 - Balao halfbeak; (3)

Family Belonidae

* $\square$ Belone belone (Linnaeus, 1761) - Garfish; Agulha (1) (3), Peixe-agulha (1) (2), Agulha(2)

Belone svetovidovi Collette \& Parin, 1970 - Short-beaked garfish; Agulha (1)

Platybelone argalus argalus (Lesueur, 1821) - Keeltail needlefish; Peixe-agulha(2)

Tylosurus acus acus (Lacepède, 1803) - Agujon needlefish; Peixe-agulha (2), Agulha (3)

Strongylura acus is a synomym of T. acus acus. Albuquerque (1954-1956) considered the presence of T. acus acus off Madeira as very doubtful. Santos et al. (1997) wrote that the identity of the species and its synonyms for the Azores needed further confirmation.

\section{Family Scomberesocidae}

* $\square$ Scomberesox saurus saurus (Walbaum, 1792) - Atlantic saury; Agulhão(1) (2), Ratinho-do-alto(2), Ratinho or Catutinho (3)

Scomberesox simulans (Hubbs \& Wisner, 1980) - Dwarf saury; (1) (2) (3)

Order Stephanoberyciformes

Family Melamphaidae

Melamphaes indicus Ebeling, 1962 - No common name; (3)

Melamphaes falsidicus Kotlyar, 2011 - No common name; (2) (3)

Kotlyar (2011) described the species M. falsidicus from the northern Atlantic Ocean, where it was sampled between $34^{\circ} \mathrm{N}$ and $58^{\circ} \mathrm{N}$. According to Kotlyar (2011), this species was previously defined as Melamphaes microps (Günther, 1878), which is probably a variant of M. falsidicus. The records of the species M. microps off the Azores and in Madeira should probably be considered as M. falsidicus.

Melamphaes polylepis Ebeling, 1962 - No common name; (2)

The species M. polylepis was recorded off the Azores, in the PECS area (10 NM north of the Maxwell Fracture Zone and 15 NM NNW of the Olympus Knoll), MAR-ECO - HamPelFish, No. 34850193, 27.8029

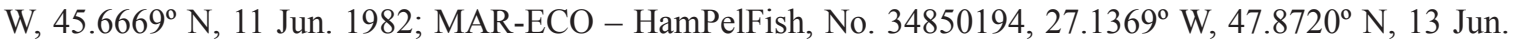
1982. Kotlyar (2011) indicated that the Atlantic population of this species is isolated from the Indian-Pacific region, and the comparative analysis of specimens sampled in different geographical regions would be quite interesting, allowing a reliable species definition.

$\square \quad$ Melamphaes longivelis Parr, 1933 - Ridgehead; (2) (3)

Melamphaes pumilus Ebeling, 1962 - No common name; (2)

This species occurs off the Azores, in the PECS area (118 NM southeast of the Cruiser Tablemount), SOC, Discovery No. 1126123 85 FIS 516001, 25.3408 W, $31.4258^{\circ}$ N, 29 Jun. 1985.

Melamphaes simus Ebeling, 1962 - Ridgehead; (1) (2) (3)

$\square \quad$ Melamphaes suborbitalis (Gill, 1883) - Ridgehead; (1), Peixe-rã(2) (3)

Melamphaes typhlops (Lowe, 1843) - Ridgehead; Peixe-rã (1) (2) (3)

* $\square$ Poromitra capito Goode \& Bean, 1883 - Ridgehead; Peixe-rã (1) (2) (3)

Poromitra crassiceps (Günther, 1878) - Crested bigscale; (2) (3) 
Poromitra megalops (Lütken, 1878) - Ridgehead; (1) (2)

This species occurs off the Portuguese mainland, in the PECS area (50 NM SSW of the Almeida Carvalho Seamount), SOC, Discovery No. 1103601_84_FIS_658002, 14.9616º W, 39.4000 N, 1 Apr. 1984.

Poromitra nigriceps (Zugmayer, 1911) - No common name; (1) (2)

Scopeloberyx opisthopterus (Parr, 1933) - Ridgehead; (1) (2) (3)

Scopeloberyx robustus (Günther, 1887) - Ridgehead; Peixe-rã (1) (2), (3)

Scopeloberyx rubriventer (Koefoed, 1953) - Ridgehead; (2)

Scopelogadus beanii (Günther, 1887) - Ridgehead; (2) (3)

Scopelogadus mizolepis mizolepis (Günther, 1878) - Bigscale; (2)

Family Stephanoberycidae

Acanthochaenus luetkenii Gill, 1884 - Prickdefish; (2)

Family Rondeletiidae

Rondeletia bicolor Goode \& Bean, 1895 - No common name; (2)

This species occurs off the Azores, in the PECS area (60 NM WNW of the Marsala Seamount), MCZ, No. 62168, 36.4483 ${ }^{\circ} \mathrm{W}, 34.9350^{\circ} \mathrm{N}, 27$ Aug. 1984.

Rondeletia loricata Abe \& Hotta, 1963 - Redmouth whalefish; (2) (3)

\section{Family Cetomimidae}

Cetichthys indagator (Rofen, 1959) - No common name; (3)

Cetomimus gillii Goode \& Bean, 1895 - No common name; (2)

This species occurs off the Azores, in the PECS area (60 NM WNW of the Marsala Seamount), MAR-ECO

- HamPelFish, No. 34847825, 35.4830 W, 34.3499 N, 28 Apr. 1979.

Cetomimus hempeli Maul, 1969 - Whalefish; (3)

Cetostoma regani Zugmayer, 1914 - Pink flabby whalefish; (1) (2) (3)

Ditropichthys storeri (Goode \& Bean, 1895) - No common name; (2)

The species D. storeri is reported to occur off the Azores, in the PECS area (107 NM southeast of the Cruiser

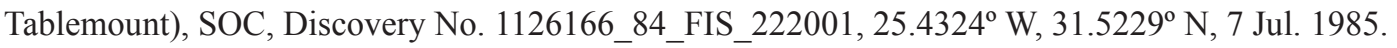

Gyrinomimus myersi Parr, 1934 - No common name; (2)

Procetichthys kreffti Paxton, 1989 - No common name; (2)

The presence of the species $P$. kreffti is reported off the Azores, in the PECS area (95 NM northwest of the

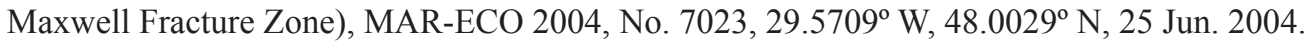

\section{Family Mirapinnidae}

Eutaeniophorus festivus (Bertelsen \& Marshall, 1956) - Festive ribbonfish; (2) (3)

This species is recorded off the Azores, in the PECS area (126 NM east of the Hyeres Seamount), SOC, Discovery No. 1126173_84_FIS_262001, 25.3366º W, 31.0083º N, 7 Jul. 1985.

Mirapinna esau Bertelsen \& Marshall, 1956 - Hairyfish; (2)

Parataeniophorus gulosus Bertelsen \& Marshall, 1956 - No common name; (1) (2) (3)

Family Megalomycteridae

Ataxolepis apus Myers \& Freihofer, 1966 - No common name; (2) 
Order Beryciformes

Family Anoplogasteridae

$\square \quad$ Anoplogaster cornuta (Valenciennes, 1833) - Common fangtooth; (1) (2) (3)

Family Diretmidae

Diretmichthys parini (Post \& Quéro, 1981) - Parin's spinyfish; (2) (3)

* Diretmus argenteus Johnson, 1864 - Silver spinyfin; (1) (2) (3)

Family Trachichthyidae

Gephyroberyx darwinii (Johnson, 1866) - Darwin's slimehead; (2), Imperatriz or Peixe-vidro (3) Hoplostethus atlanticus Collett, 1889 - Orange roughy; Olho-de-vidro-laranja (1), Olho-de-vidro or Relógio(2)

Hoplostethus cadenati Quéro, 1974 - Black slimehead; Olho-de-vidro-preto(1)

* Hoplostethus mediterraneus mediterraneus Cuvier, 1829 - Mediterranean slimehead; Olho-devidro (1) (2), Relógio (2), Alfonsim-do-alto (3)

\section{Family Berycidae}

* B Beryx decadactylus Cuvier, 1829 - Alfonsino; Imperador(1) (2), Alfonsim(2), Alfonsim-da-costalarga (3)

* $\square$ Beryx splendens Lowe, 1834 - Splendid alfonsino; Imperador-de-costa-estreita (1), Alfonsim(2), Alfonsim-da-costa-estreita (3)

Family Holocentridae

Sargocentron hastatum (Cuvier, 1829) - Red squirrelfish; Esquilo-real (1)

Order Zeiformes

Family Oreosomatidae

Allocyttus verrucosus (Gilchrist, 1906) - Warty dory; (2)

The species A. verrucosus has been reported from the north of the Azores (Hareide \& Garnes 2001), in the PECS area (Mid-Atlantic Ridge, $43^{\circ}-44^{\circ} \mathrm{N}$ ).

$\square \quad$ Neocyttus helgae (Holt \& Byrne, 1908) - False boarfish, (2) (3)

Neocyttus rhomboidalis Gilchrist, 1906 - Spiky oreo; (3)

Albuquerque (1954-1956) mentioned the capture of a specimen to the north of Madeira (Maul 1948c, 1949), identified as $N$. rhomboidalis. Nevertheless, this specimen is distinct from the other specimens named by other authors. In the North Atlantic, $N$. rhomboidalis is replaced by $N$. helgae, ranging southward to Madeira. The possible occurrence of $N$. rhomboidalis in this area, as reported by Quéro (1982), seems doubtful.

\section{Family Parazenidae}

* Cyttopsis rosea (Lowe, 1843) - Rosy dory; Galo-de-natura (1) (2), Peixe-galo (2) (3), Peixe-galo-denatura (3)

Family Zeniontidae (Zenionidae)

* Zenion hololepis (Goode \& Bean, 1896) - No common name; Galito-rosado (1) 
Family Grammicolepididae

* Grammicolepis brachiusculus Poey, 1873 - Thorny tinselfish; (1) (2)

Family Zeidae

$* \diamond \square$ Zenopsis conchifer (Lowe, 1852) - Silvery John dory; Galo-branco (1) (2), Peixe-galo (2), Peixe-galode-natura (3)

* Zeus faber Linnaeus, 1758 - John dory; Galo-negro(1), Peixe-galo (1) (2) (3)

\section{Order Gasterosteiformes}

Family Gasterosteidae

* Gasterosteus aculeatus aculeatus Linnaeus, 1758 - Three-spined stickleback; Esgana-gata(1)

* Spinachia spinachia (Linnaeus, 1758) - Sea stickleback; Esgana-gata-marinha (1)

Family Syngnathidae

* Entelurus aequoreus (Linnaeus, 1758) - Snake pipefish; Cavalo-marinho (1) (2)

$\diamond \quad$ Hippocampus erectus Perry, 1810 - Lined seahorse; Cavalo-marinho (2)

* Hippocampus guttulatus Cuvier, 1829 - Long-snouted seahorse; (1), Cavalo-marinho(2) (3), Cavalinho-do-mar(3)

* $\triangleright$ Hippocampus hippocampus (Linnaeus, 1758) - Short-snouted seahorse; Cavalo-marinho (1) (2) (3)

* Nerophis lumbriciformis (Jenyns, 1835) - Worm pipefish; Marinha (1), (2)

The species $N$. lumbriciformis is regarded as being present off the Azores (records in OBIS). The specimen was caught outside the EEZ (155 NM southwest of the Georgiy Zima Seamount) and is deposited in the Muséum National d'Histoire Naturelle at Paris, MNHN, No. $0000-6074,20.0^{\circ} \mathrm{W}, 40.0^{\circ} \mathrm{N}$. This appears to be a very doubtful record, which needs confirmation.

Nerophis maculatus Rafinesque, 1810 - No common name; Cavalo-marinho(1), (2)

* $\square$ Nerophis ophidion (Linnaeus, 1758) - Straightnosed pipefish; Cavalo-marinho (1), (3)

* Syngnathus abaster Risso, 1827 - Black-striped pipefish; (1) (2)

The species $S$. abaster is regarded as being present off the Azores (records in OBIS). The specimen was caught outside the EEZ (155 NM southwest of the Georgiy Zima Seamount) and is deposited in Muséum National d'Histoire Naturelle at Paris, MNHN, No. $1955-0044,20.0^{\circ} \mathrm{W}, 40.0^{\circ} \mathrm{N}$. This appears to be a very doubtful record, which needs confirmation.

* $\square$ Syngnathus acus Linnaeus, 1758 - Great pipefish; Marinha-comum(1) (2), Chicote(2), Agulhinha (3) Syngnathus pelagicus Linnaeus, 1758 - No common name; (2)

This species was recorded from the Azores, in the PECS area (50 NM north and 87 NM west of the Atlantis Seamount, $45 \mathrm{NM}$ to the northwest of the Konstantinov Ridge), ZMUC, No. $310,30.0^{\circ} \mathrm{W}, 35.0^{\circ} \mathrm{N}$; ZMUC, No. $312,30.0^{\circ} \mathrm{W}, 35.0^{\circ} \mathrm{N}$; ZMUC, No. $357,34.4200^{\circ} \mathrm{W}, 32.2700^{\circ} \mathrm{N}$; ZMUC, No. $381,32.0^{\circ} \mathrm{W}, 34.0^{\circ} \mathrm{N}$; ZMUC, No. $384,32.0^{\circ} \mathrm{W}, 39.0^{\circ} \mathrm{N}$.

Syngnathus phlegon Risso, 1827 - No common name; (1)

* Syngnathus rostellatus Nilsson, 1855 - Nilsson's pipefish; Marinha-cabeça-chata (1)

* Syngnathus typhle Linnaeus, 1758 - Deep-snouted pipefish; Marinha-focinho-grosso (1)

Family Aulostomidae

$\square \quad$ Aulostomus strigosus Wheeler, 1955 - Atlantic cornetfish; Trombeta (3) 
Family Fistulariidae

$* \diamond \square$ Fistularia petimba Lacepède, 1803 - Red cornetfish; Corneta-rosada (1), (2)

New record for the Portuguese mainland waters, the first specimen of $F$. petimba (Fig. 3) (1150 mm TL and $555 \mathrm{~g}$ ) was caught by fishermen from Sesimbra, using trammel nets (100 $\mathrm{mm}$ mesh size, at lint), at a depth of $48 \mathrm{~m}$, on the $22^{\text {th }}$ of January $2008\left(38^{\circ} 27.096^{\prime} \mathrm{N}, 9^{\circ} 14.284^{\prime} \mathrm{W}\right)$.

Fistularia tabacaria Linnaeus, 1758 - Cornetfish; Corneta-malhada, (2)

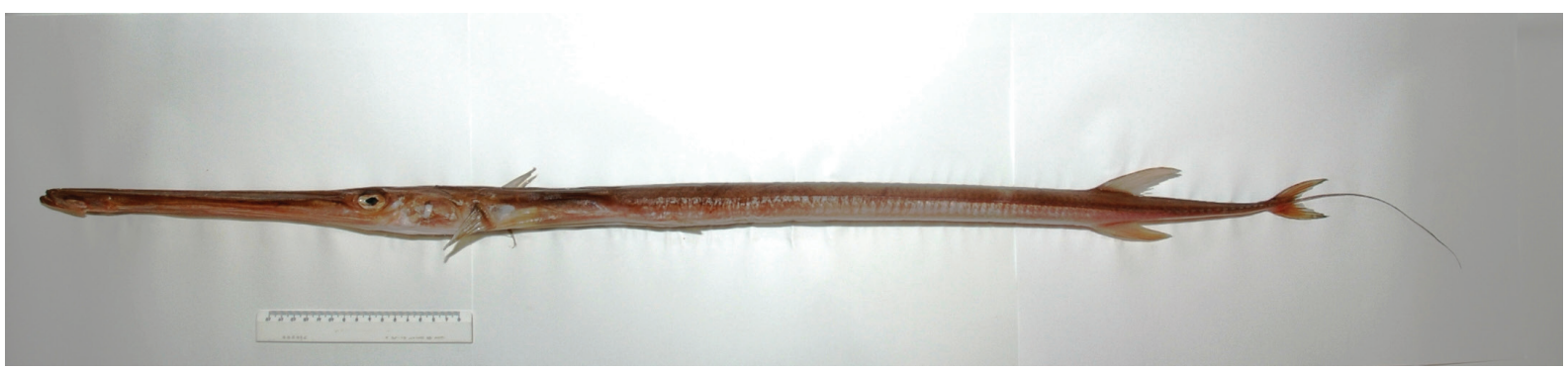

Fig. 3. Fistularia petimba Lacepède, 1803.

Family Macroramphosidae

* $\square$ Macroramphosus gracilis (Lowe, 1839) - Slender snipefish; Trombeteiro (1) (2) (3)

* Macroramphosus scolopax (Linnaeus, 1758) - Longspine snipefish; Trombeteiro (1) (2) (3), Peixetrombeteiro(2)

Robalo et al. (2009) suggested that the genus Macroramphosus is represented by a single species in the northeastern Atlantic, M. scolopax, with different morphotypes interbreeding off the central and southwestern Portuguese coast. Dyer \& Westneat (2010) indicated the distribution of the species M. gracilis as being the western Central Atlantic and this species seems to be sympatric with M. scolopax, all around the world.

\section{Order Scorpaeniformes}

Family Dactylopteridae

* Dactylopterus volitans (Linnaeus, 1758) - Flying gurnard; Cabrinha-de-leque(1), (2) (3) Wirtz el al. (2008) considered this as a doubtful record for Madeira.

\section{Family Scorpaenidae}

* Helicolenus dactylopterus dactylopterus (Delaroche, 1809) - Blackbelly rosefish; Cantarilholegítimo (1), Boca-negra (2) (3)

Phenacoscorpius nebris Eschmeyer, 1965 - No common name; (3)

This species is recorded off Madeira, in the PECS area (Josephine Bank), SDSC, No. 16587, $14.2266^{\circ} \mathrm{W}$, $36.7183^{\circ} \mathrm{N}$.

* Pontinus kuhlii (Bowdich, 1825) - Offshore rockfish; Cantarilho-requeime (1), Brage, Cantarilho or Cântaro (2), Requeme or Vermelho(3)

Scorpaena azorica Eschmeyer, 1969 - No common name; (2)

- Scorpaena canariensis (Sauvage, 1878) - No common name; (2) (3)

* Scorpaena elongata Cadenat, 1943 - Slender rockfish; Rascasso-rosado(1) Scorpaena laevis Troschel, 1866 - Senegalese rockfish; (2) (3)

$\square \quad$ Scorpaena loppei Cadenat, 1943 - Cadenat's rockfish; (1) (2) (3) 
S. loppei occurs off the Azores (Great Meteor Tablemount) and off Madeira (Josephine Bank), in the PECS area, SDSC, No. $23054,28.4966^{\circ} \mathrm{W}, 29.8366^{\circ} \mathrm{N}$; SDSC, No. $23056,28.5250^{\circ} \mathrm{W}, 30.0500^{\circ} \mathrm{N}$; SDSC, No. $23055,28.4000^{\circ} \mathrm{W}, 30.0183^{\circ} \mathrm{N}$; SDSC, No. $23052,14.2533^{\circ} \mathrm{W}, 36.7050^{\circ} \mathrm{N}$; SDSC, No. $23053,14.2916^{\circ} \mathrm{W}$, $36.6700^{\circ} \mathrm{N}$.

$\square \quad$ Scorpaena maderensis Valenciennes, 1833 - Madeira rockfish; (1), Rascasso or Coça(2), Rocaz(3)

* $\square$ Scorpaena notata Rafinesque, 1810 - Small red scorpionfish; Rascasso-escorpião(1), Rascasso or Coça(2), (3)

Scorpaena plumieri Bloch, 1789 - Pacific spotted scorpionfish; (2) (3)

* Scorpaena porcus Linnaeus, 1758 - Black scorpionfish; Rascasso-de-pintas(1), Rascasso or Coça (2), (3)

* $\square$ Scorpaena scrofa Linnaeus, 1758 - Red scorpionfish; Rascasso-vermelho(1), Rocaz(2), Palhaço or Peixe-carneiro (3)

Scorpaena stephanica Cadenat, 1943 - Spotted-fin rockfish; (3)

This species occurs off Madeira, in the PECS area (Josephine Bank), SOC, Discovery No. 785703_210_ FIS_766003, $14.3033^{\circ} \mathrm{W}, 36.7391^{\circ} \mathrm{N}, 11$ Apr. 1972.

$\diamond \quad$ Scorpaenodes arenai Torchio, 1962 - No common name; (2)

* $\square$ Setarches guentheri Johnson, 1862 - Channeled rockfish; (1) (2), Requeme-de-natura (3)

Trachyscorpia cristulata cristulata (Goode \& Bean, 1896) - Atlantic thornyhead; (1)

$\diamond \square$ Trachyscorpia cristulata echinata (Köhler, 1896) - Spiny scorpionfish, (1) (2) (3)

\section{Family Triglidae}

* Chelidonichthys cuculus (Linnaeus, 1758) - Red gurnard; Cabra-vermelha(1), Cabra (2) (3), Cabrinha or Ruivo(2)

* $\square$ Chelidonichthys lucerna (Linnaeus, 1758) - Tub gurnard; Cabra-cabaço(1), (3)

* $\square$ Chelidonichthys obscurus (Walbaum, 1792) - Longfin gurnard; Cabra-de-bandeira (1), (2) (3)

* Eutrigla gurnardus (Linnaeus, 1758) - Grey gurnard; Cabra-morena (1), (3)

* Lepidotrigla cavillone (Lacepède, 1801) - Large-scaled gurnard; Ruivo(1)

* Lepidotrigla dieuzeidei Blanc \& Hureau, 1973 - Spiny gurnard; Ruivo-espinhoso(1)

* $\square$ Trigla lyra Linnaeus, 1758 - Piper gurnard; Cabra-lira (1), Cabra (3)

Santos et al. (1997) indicated that the record of T. lyra from the Azores is based on a probable misidentification of Chelidonichthys cuculus (Linnaeus, 1758) by Sampaio (1904). The presence of this species off the Azores needs further documentation.

* Trigloporus lastoviza (Bonnaterre, 1788) - Straked gurnard; Cabra-riscada(1), (2), Cabrinha or Cabra (3)

\section{Family Peristediidae}

* Peristedion cataphractum (Linnaeus, 1758) - African armoured searobin; Cabra-de-casca (1), (2) (3)

Family Cottidae

Myoxocephalus scorpius (Linnaeus, 1758) - Shorthorn sculpin; Escorpião(1)

Nobre (1935) and Albuquerque (1954-1956) indicated the presence of this species off the Portuguese mainland, considering it as rare.

* Taurulus bubalis (Euphrasen, 1786) - Longspined bullhead; Escorpião-roco(1)

Family Psychrolutidae

Cottunculus thomsonii (Günther, 1882) - Pallid sculpin; (1) 
Family Cyclopteridae

Cyclopterus lumpus Linnaeus, 1758 - Lumpfish; Peixe-lapa (1)

Eumicrotremus spinosus (Fabricius, 1776) - Atlantic spiny lumpsucker; (2)

Family Liparidae

Paraliparis copei copei Goode \& Bean, 1896 - No common name; (2)

Paraliparis membranaceus Günther, 1887 - No common name

The holotype of $P$. membranaceus was caught on the $10^{\text {th }}$ of January 1876, off Cabo San Vicente, Sarmiento

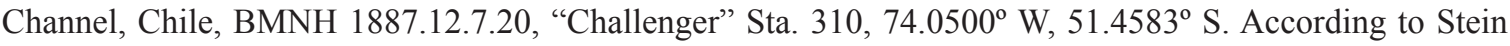
(2005), information on the collection area was obscure but unequivocal (Murray 1895). Over time, erroneous reports of the occurrence of P. membranaceus accumulated in literature. In fact, Günther's (1887) description omitted mentioning that it was from Chile (“... off Cape St. Vincent, Station 310 ...”). Subsequent authors did not verify the actual collection data, and even the Natural History Museum online catalogue included incorrect collection data that have subsequently been corrected (Stein 2005). Burke (1930) also omitted the information that the specimen was from the fjord waters of southern Chile, and Nobre (1935) and Albuquerque (1954-1956) referred to Günther and Burke. Still later, Lindberg (1973) misinterpreted "Cape St. Vincent" as meaning "off Portugal", i.e. the Cape St. Vincent, which is the best known geographic feature of the south coast of Portugal. Stein \& Able (1986) cited Lindberg. The species P. membranaceus was omitted from the reviews of Chilean liparids (Stein et al. 1991) and of Southern Ocean species (Andriashev 1998, 2003). The P. membranaceus record off mainland Portugal is erroneous. For this reason this species is not included in this list.

Paraliparis nigellus Chernova \& Møller, 2008 - No common name; (2)

\section{Order Perciformes \\ Family Howellidae}

Prokofiev (2006) suggested that Howella, Pseudohowella and Bathysphyraenops should be placed in the family Howellidae, which contains three genera from the Percichthyidae and the Acropomatidae. Prokofiev $(2007 \mathrm{a}, \mathrm{b})$ discussed the systematic position of the genus Howella and revised the diagnosis of the family Howellidae. According to Eschmeyer (2013), the family contains nine valid species.

Bathysphyraenops simplex Parr, 1933 - No common name; (1)

Howella atlantica Post \& Quéro, 1991 - No common name; (1) (2) (3)

Post \& Quéro (1991) reviewed the species Howella brodiei Ogilby, 1899, and demonstrated the existence of two subspecies: H. brodiei atlantica (Post \& Quéro, 1991) from the North and tropical Atlantic and $H$. brodiei brodiei Ogilby, 1899 from the Indian and Pacific Oceans. The authors also showed that the number of scales between the origin of the second dorsal fin and the lateral line separates the Atlantic populations of Howella into two different species: Howella brodiei atlantica and Howella sherborni (Norman, 1930). Eschmeyer (2013) raised the subspecies Howella brodiei atlantica (Post \& Quéro, 1991) to species level: Howella atlantica (Post \& Quéro, 1991). The records of H. brodiei in the northeast Atlantic should probably be considered as belonging to $H$. atlantica .

$\square \quad$ Howella sherborni (Norman, 1930) - No common name; (2) (3)

This species occurs off the Azores, in the PECS area (72 NM southwest of the Albany Seamount).

Family Moronidae

* Dicentrarchus labrax (Linnaeus, 1758) - European seabass; Robalo-legítimo or Robalo(1)

* Dicentrarchus punctatus (Bloch, 1792) - Spotted seabass; Robalo-baila or Baila (1) 
Family Polyprionidae

* $\square$ Polyprion americanus (Bloch \& Schneider, 1801) - Wreckfish; Cherne(1) (2) (3)

Family Serranidae

* $\square$ Anthias anthias (Linnaeus, 1758) - Swallowtail seaperch; Canário-do-mar(1) (2), Folião or Piriquito (2), Castanheta-do-alto or Imperador(3)

Epinephelus aeneus (Geoffroy Saint-Hilaire, 1817) - White grouper; Garoupa-legítima (1)

Epinephelus caninus (Valenciennes, 1843) - Dogtooth grouper; Mero-gigante (1)

Epinephelus costae (Steindachner, 1878) - Goldblotch grouper; (1)

* Epinephelus marginatus (Lowe, 1834) - Dusky grouper; Mero (1) (2) (3)

ㅁ Mycteroperca fusca (Lowe, 1838) - Island grouper; Badejo (2) (3)

Mycteroperca phenax Jordan \& Swain, 1884 - Scamp; (2)

Mycteroperca rubra (Bloch, 1793) - Mottled grouper; Garoupa-chumbo(1)

* $\square$ Serranus atricauda Günther, 1874 - Blacktail comber; Serrano-de-rolo (1), Garoupa (2) (3)

* $\square$ Serranus cabrilla (Linnaeus, 1758) - Comber; Serrano-alecrim(1), Garoupa-do-alto (2), Garoupa (3)

* Serranus hepatus (Linnaeus, 1758) - Brown comber; Serrano-ferreiro(1)

Serranus scriba (Linnaeus, 1758) - Painted comber; Serrano-riscado(1), (2) (3)

Family Callanthiidae

$* \diamond \square$ Callanthias ruber (Rafinesque, 1810) - Parrot seaperch; Canarinho-do-mar(1), Papagaio(2), Castanheta-amarela-do-alto(2) (3)

Family Priacanthidae

$\diamond \quad$ Cookeolus japonicus (Cuvier, 1829) - Longfinned bullseye; (2)

$\square \quad$ Heteropriacanthus cruentatus (Lacepède, 1801) - Glasseye; Fura-vasos-da-rocha, Fura-vasos (3)

$\diamond \quad$ Priacanthus arenatus Cuvier, 1829 -Atlantic bigeye; Fura-vasos-vulgar or Catalufa(2), (3)

Family Apogonidae

$\square \quad$ Apogon imberbis (Linnaeus, 1758) - Cardinal fish; Alcarraz (1) (2), Folião or Cardeal(2), Alfonsinhoda-Costa or Alcaraz (3)

\section{Family Epigonidae}

Epigonus constanciae (Giglioli, 1880) - No common name; (2) (3)

Epigonus denticulatus Dieuzeide, 1950 - Pencil cardinal; (1) (2)

The species E. denticulatus is regarded as being present to the north of the Azores (Hareide \& Garnes 2001), in the PECS area (Mid-Atlantic Ridge, $43^{\circ}-44^{\circ} \mathrm{N}$ ).

$* \square$ Epigonus telescopus (Risso, 1810) - Black cardinal fish; Olhudo(1), Escamuda, Escuro, Jordão or Preto (2), Robaldo, Robaldo-do-alto or Robalo-preto (3)

Microichthys coccoi Rüppell, 1852 - No common name; (2)

Family Pomatomidae

* $\square$ Pomatomus saltatrix (Linnaeus, 1766) - Bluefish; Anchova (1) (2), Enchova(2) (3) 
Family Coryphaenidae

$\square \quad$ Coryphaena equiselis Linnaeus, 1758 - Pompano dolphinfish; Doirado-pampo(1), Dourado(1) (2), Doirado or Doirado-amarelo (2), Dourada or Delfim (3)

$\square \quad$ Coryphaena hippurus Linnaeus, 1758 - Common dolphinfish; Doirado(1) (2), Dourado(2) (3)

Family Echeneidae

* $\triangleright$ Echeneis naucrates Linnaeus, 1758 - Live sharksucker; Rémora (1) (2) (3), Agarrador or Pegador(2)

* Phtheirichthys lineatus (Menzies, 1791) - Slender suckerfish; (1), Agarrador, Pegador or Rémora (2)

$\square \quad$ Remora albescens (Temminck \& Schlegel, 1850) - White suckerfish; (1), Agarrador, Rémora (2), Pegador(2) (3), Chupa-sangue (3)

* Remora brachyptera (Lowe, 1839) - Spearfish remora; Pegador(1) (2) (3), Agarrador(2), Chupasangue (3)

$* \diamond \square$ Remora osteochir (Cuvier, 1829) - Marlinsucker; (1), Agarrador, Pegador or Rémora (2), (3)

* Remora remora (Linnaeus, 1758) - Sharksucker; Pegador(1), Rémora(1) (2) (3), Agarrador(2), Pegador(2) (3)

\section{Family Carangidae}

The limits of carangid genera are in many cases poorly defined. This is in part a consequence of some fundamental problems in the delimitation of generic and sub-generic taxa within the family (Gunn 1990). A particular case in point is the separation of the large genus Caranx Lacepède into a series of smaller genera including Carangoides Bleeker, Selar Bleeker, Alepes Swainson, Atule Jordan \& Jordan, Decapterus Sleeker, Pseudocaranx Bleeker, Ulua Jordan \& Snyder, Uraspis Bleeker and Caranx (sensu stricto) (Gunn 1990).

Alectis alexandrinus (Geoffroy Saint-Hilaire, 1817) - Alexandria pompano; Xareu-enxada (1) Campogramma glaycos (Lacepède, 1801) - Vadigo; Xareu-palheta (1), (3)

- Caranx crysos (Mitchill, 1815) - Blue runner; Xareu-azul (1) (2), Írio-de-serra or Xareu-gacesse (2), (3)

$\square \quad$ Caranx hippos (Linnaeus, 1766) - Crevalle jack; Xareu-macoa (1), (2), Charéu or Encharéu-denatura (3)

$\square \quad$ Caranx latus Agassiz, 1831 - Horse-eye jack; (3)

$\square \quad$ Caranx lugubris Poey, 1860 - Black jack; (2), Encharéu or Charéu (3) Caranx rhonchus Geoffroy Saint-Hilaire, 1817 - False scad; Charro-amarelo(1), (3) This record of this species from off Madeira needs verification (Wirtz et al. 2008).

Caranx ruber (Bloch, 1793) - Bar jack; Xareu-carvoeiro, (2)

Carangoides ruber (Bloch, 1793) is synonym of Caranx ruber, which some sources (ITIS) still cite as valid.

$\square \quad$ Caranx sexfasciatus Quoy \& Gaimard, 1825 - Bigeye trevally; Charéu-de-natura or Encharéu (3)

The species C. sexfasciatus is referred to as being present off Madeira by Maul (1948a), with the indication of very rare occurrence. There are an additional twelve records of the species in the Atlantic Ocean. According to Nichols (1938), C. sexfasciatus is a circumtropical species. Hureau \& Tortonese (1979) reported that Maul's identification might be questionable.

$\diamond \square$ Decapterus macarellus (Cuvier, 1833) - Mackerel scad; Cavala-preta(2), (3)

- Decapterus punctatus (Cuvier, 1829) - Round scad; Charro-moiro, (2) (3)

This species is recorded off the Azores, in the PECS area (87 NM west of the Great Meteor Tablemount), ROM, No. 23918, 30.2500 W, 30.2916 N, 29 Jan. 1966.

$\diamond$ Elagatis bipinnulata (Quoy \& Gaimard, 1825) - Rainbow runner; Fogueteiro-arco-iris(1) (2), Salemão(2)

Lichia amia (Linnaeus, 1758) - Leerfish; Palombeta or Doirada (1), (2) (3)

* $\quad$ Naucrates ductor (Linnaeus, 1758) - Pilotfish; Peixe-piloto (1) (2), Romeiro (1) (2) (3) 
$\square \quad$ Pseudocaranx dentex (Bloch \& Schneider, 1801) - White trevally; Xareu-bicudo or Xaréu(2), Encharéu(2) (3), Charéu (3)

Selene dorsalis (Gill, 1863) - African moonfish; Corcovado-africano(1), (3)

$\square \quad$ Seriola dumerili (Risso, 1810) - Greater amberjack; Charuteiro-catarino(1), Írio or Lírio(2), Charuteiro (3)

$\square \quad$ Seriola fasciata (Bloch, 1793) - Lesser amberjack; (2), Charuteiro(3)

$\square \quad$ Seriola lalandi Valenciennes, 1833 - Yellowtail amberjack; Enchova (1), Charuteiro (3)

- Seriola rivoliana Valenciennes, 1833 - Longfin yellowtail; (1), Írio or Lírio (2), Charuteiro(3)

* Trachinotus carolinus (Linnaeus, 1766) - Florida pompano; Sereia-da-Florida, (2)

* Trachinotus ovatus (Linnaeus, 1758) - Pompano; Sereia-camochilo(1), Cabra, Plombeta or Prombeta (2), Trombeta, Facaio or Ranhosa (3)

Trachurus lathami Nichols, 1920 - Rough scad; Carapau-rugoso, (2)

Very rare off the Azores, with only one record, MCZ, No. 166359, $23.9166^{\circ} \mathrm{W}, 36.6000^{\circ} \mathrm{N}, 26$ Jun. 1969.

* Trachurus mediterraneus (Steindachner, 1868) - Mediterranean horse mackerel; Carapau-doMediterrâneo (1)

* $\square$ Trachurus picturatus (Bowdich, 1825) - Blue jack mackerel; Carapau-negrão(1), Chicharro-doalto(2), Chicharro (2) (3)

* $\square$ Trachurus trachurus (Linnaeus, 1758) - Atlantic horse mackerel; Carapau(1), (2), Chicharrocharéu(3)

Family Bramidae

* Brama brama (Bonnaterre, 1788) - Atlantic pomfret; Xaputa (1), Chaputa (2), Freira (1) (2) (3)

Pteraclis carolinus Valenciennes, 1833 - Fanfish; (2), Freira or Xaputa (3)

There are records of the species $P$. carolinus off the Azores, in the PECS area (90 NM northwest of the Hyeres Seamount and 44 NM west of the Oceanographer Fracture Zone), MCZ, No. $76279,30.6166^{\circ} \mathrm{W}, 31.8333^{\circ} \mathrm{N}$,

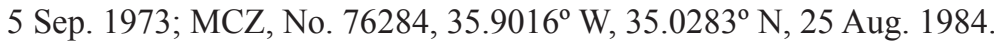

$\diamond \quad$ Pterycombus brama Fries, 1837 - Atlantic fanfish; (1) (2) (3)

$\square \quad$ Taractes asper Lowe, 1843 - Rough pomfret; (1) (2) (3)

Taractes rubescens (Jordan \& Evermann, 1887) - Dark pomfret; (2) (3)

$\diamond \square$ Taractichthys longipinnis (Lowe, 1843) - Big-scale pomfret; Xaputa-galhuda(1) (2), Xaputa(2), Freira-de-natura (3)

\section{Family Caristiidae}

Caristius macropus (Bellotti, 1903) - Manefish; (2)

This species is recorded off the Azores, in the PECS area (112 NM south of the Lucky Strike Seamount, 11 NM and 54 NM north of the Maxwell Fracture Zone), MAR-ECO - HamPelFish, No. 34843840, $32.0169^{\circ} \mathrm{W}$, $35.4000^{\circ}$ N, 29 Apr. 1979; MAR-ECO - HamPelFish, No. 34843841, $27.1369^{\circ}$ W, $47.8720^{\circ}$ N, 13 Jun. 1982; MAR-ECO - HamPelFish, No. $34843842,27.5330^{\circ} \mathrm{W}, 48.5880^{\circ} \mathrm{N}$.

$\square \quad$ Paracaristius maderensis (Maul, 1949) - No common name; (2) (3)

Platyberyx mauli Kukuev, Parin \& Trunov, 2012 - No common name; (3)

According to Kukuev et al. (2012), the specimen described by Maul in 1949 as Platyberyx opalescens Zugmayer, 1911 concerns $P$. mauli. The latter differs from $P$. opalescens in the structure of a branchial apparatus (morphology and number of gill rakers and more numerous teeth on the vomer) and body proportions (body depth, eye diameter, head length and position of the pelvic fins).

- Platyberyx opalescens Zugmayer, 1911 - No common name; Aia(1), (2) (3)

According to Kukuev et al. (2012), reliable catches of $P$. opalescens are known from Portugal to northern Mauritania and suggest that the range of the species includes the near-bottom waters of the continental slope (bathial-pelagic ichthyocene) of the East Atlantic. According to Santos et al. (1997), the presence of this species off the Azores is based on the identification of a $63 \mathrm{~mm}$ specimen caught by the R/V "Michael Sars" at 
$29.7833^{\circ} \mathrm{W}, 36.8833^{\circ} \mathrm{N}$ (St. 56). According to Eschmeyer (2013) the range of the species is wider (Greenland and eastern Atlantic).

Family Lutjanidae

Etelis carbunculus Cuvier, 1828 - Deep-water red snapper; Vermelho-salmão (3)

The species E. carbunculus is referenced for Madeira by Maul (1948a). No other records have been reported from the Atlantic Ocean.

Etelis oculatus (Valenciennes, 1828) - Queen snapper; (3)

This species is reported from Madeira, based on a single record kept at the Natural History Museum (BMNH 1857.6.13.112, identified as Serranus oculatus Valenciennes, 1828). S. oculatus is a synonym E. oculatus.

Lutjanus goreensis (Valenciennes, 1830) - Gorean snapper; Luciano-da-Goreia, (3)

Rhomboplites aurorubens (Cuvier, 1829) - Vermilion snapper; (2)

$R$. aurorubens is recorded off the Azores, in the PECS area (82 NM west of the Great Meteor Tablemount), ROM, No. 23910, $30.2500^{\circ} \mathrm{W}, 30.2916^{\circ} \mathrm{N}, 29$ Jan. 1966.

\section{Family Lobotidae}

Lobotes surinamensis (Bloch, 1790) - Tripletail; Furriel(1) (2), Lobotes or Peixe-folha (2), (3)

Family Haemulidae

* Parapristipoma humile (Bowdich, 1825) - Guinean grunt; Roncador-canela (1), (3)

- Parapristipoma octolineatum (Valenciennes, 1833) - African striped grunt; Riscado(1), (3)

Plectorhinchus mediterraneus (Guichenot, 1850) - Rubberlip grunt; Pombo (1)

$* \diamond \square$ Pomadasys incisus (Bowdich, 1825) - Bastard grunt; Roncador-bravura(1), Roncador(1) (3), Ronqueirão(2), Músico(3),

\section{Family Sparidae}

* $\square$ Boops boops (Linnaeus, 1758) - Bogue; Boga-do-mar(1), Boga (2) (3)

Dentex canariensis Steindachner, 1881 - Canary dentex; Dentão-das-Canárias (1)

* Dentex dentex (Linnaeus, 1758) - Common dentex; Capatão-legítimo (1), (2) (3)

$\square \quad$ Dentex gibbosus (Rafinesque, 1810) - Pink dentex; Capatão-de-bandeira (1), (3)

* Dentex macrophthalmus (Bloch, 1791) - Large-eye dentex; Cachucho(1)

Dentex maroccanus Valenciennes, 1830 - Morocco dentex; Cachucho-dentão or Dentão-deMarrocos (1)

* Diplodus annularis (Linnaeus, 1758) - Annular seabream; Sargo-alcorraz(1), (3)

* Diplodus bellottii (Steindachner, 1882) - Senegal seabream; Sargo-do-Senegal or Mucharra (1)

$\square \quad$ Diplodus cervinus cervinus (Lowe, 1838) - Zebra seabream; Sargo-veado (1) (3) Diplodus puntazzo (Walbaum, 1792) - Sharpsnout seabream; Sargo-bicudo(1)

Diplodus sargus cadenati de la Paz, Bauchot \& Daget, 1974 - Moroccan white seabream; Sargolegítimo (1), Sargo (2), (3)

Diplodus sargus sargus (Linnaeus, 1758) - White seabream; Sargo-legítimo-do-Mediterrâneo(1), (2), Sargo or Sargo-branco (3)

$* \diamond \square$ Diplodus vulgaris (Geoffroy Saint-Hilaire, 1817) - Common two-banded seabream; Sargo-safia (1), (2), Seifia (3)

* Lithognathus mormyrus (Linnaeus, 1758) - Sand steenbras; Ferreira (1) (3), Riscado (3)

* $\square$ Oblada melanura (Linnaeus, 1758) - Saddled seabream; Dobradiça (1), Dobrada (3)

* Pagellus acarne (Risso, 1827) - Axillary seabream; Besugo (1) (2) (3)

Pagellus bellottii Steindachner, 1882 - Red pandora; Bica-buço(1) 
* $\square$ Pagellus bogaraveo (Brünnich, 1768) - Blackspot seabream; Goraz(1) (2) (3), Carapau or Peixão(2)

$* \square$ Pagellus erythrinus (Linnaeus, 1758) - Common Pandora; Bica (1) (3), (2)

* Pagrus auriga Valenciennes, 1843 - Redbanded seabream; Pargo-sêmola (1), (3)

Pagrus caeruleostictus (Valenciennes, 1830) - Bluespotted seabream; Pargo-ruço (1)

* Pagrus pagrus (Linnaeus, 1758) - Red porgy; Pargo-legítimo (1), Pargo (1) (2) (3)

* $\square$ Sarpa salpa (Linnaeus, 1758) - Salema; Salema (1) (2) (3)

* Sparus aurata Linnaeus, 1758 - Gilthead seabream; Dourada (1), (2) (3)

* $\square$ Spondyliosoma cantharus (Linnaeus, 1758) - Black seabream; Choupa (1) (3), (2)

\section{Family Centracanthidae}

$* \diamond \square$ Centracanthus cirrus Rafinesque, 1810 - Curled picarel; Boqueirão (1) (2) (3)

* Spicara maena (Linnaeus, 1758) - Blotched picarel; Trombeiro-choupa (1) Spicara melanurus (Valencienneas, 1830) - Blackspot picarel; Trombeiro-malha-redonda, (3) Spicara smaris (Linnaeus, 1758) - Picarel; Trombeiro-boga (1)

Family Sciaenidae

* Argyrosomus regius (Asso, 1801) - Meagre; Corvina-legítima (1), (2) Sciaena umbra Linnaeus, 1758 - Brown meagre; Roncadeira-preta (1), (3) Umbrina canariensis Valenciennes, 1843 - Canary drum; Calafate-das-Canárias (1), (3) Umbrina cirrosa (Linnaeus, 1758) - Shi drum; Calafate-de-riscas (1) Umbrina ronchus Valenciennes, 1843 - Fusca drum; (1) (3)

\section{Family Mullidae}

* Mullus barbatus barbatus Linnaeus, 1758 - Red mullet; Salmonete-da-vasa (1), Salmonete(2) (3)

* $\square$ Mullus surmuletus Linnaeus, 1758 - Surmullet; Salmonete-legítimo(1), Salmonete(2), Salmonetevermelho(2) (3), Salmonete-do-rolo (3)

\section{Family Kyphosidae}

Kyphosus incisor (Cuvier, 1831) - Yellow sea chub; Patruça, Preguiçosa or Salema-do-Brasil(2), (3) $\square \quad$ Kyphosus sectatrix (Linnaeus, 1758) - Bermuda sea chub; Preguiçosa-branca (1), Patruça or Salemado-Brasil(2), Preguiçosa(2) (3)

\section{Family Chaetodontidae}

Chaetodon ocellatus Bloch, 1787 - No common name; (2) This species is recorded off the Azores, in the PECS area (88 NM WNW of the Great Meteor Tablemount), ROM, No. 23917, 30.2500 ${ }^{\circ}$ W, $30.2916^{\circ}$ N, 29 Jan. 1966.

Chaetodon sedentarius Poey, 1860 - Reef butterflyfish; Peixe-borboleta(2)

Family Cepolidae

* Cepola macrophthalma (Linnaeus, 1758) - Red bandfish; Suspensório(1)

Family Pomacentridae

$\square \quad$ Abudefduf luridus (Cuvier, 1830) - Canary damsel; (1), Castanheta-preta(2) (3), Castanheta-ferreira (3)

$\square \quad$ Abudefduf saxatilis (Linnaeus, 1758) - Sergeant-major; (3)

* $\square$ Chromis chromis (Linnaeus, 1758) - Damselfish; Castanheta (1) (3) 
- Chromis limbata (Valenciennes, 1833) - Azores chromis; Castanheta-amarela (2), Castanheta-báia or Castanheta-branca (3)

\section{Family Labridae}

* Acantholabrus palloni (Risso, 1810) - Scale-rayed wrasse; Bodião-do-alto (1) (2), Truta-do-alto(3)

$\square \quad$ Bodianus scrofa (Valenciennes, 1839) - Barred hogfish; Bodião-dente-de-cão, Peixe-cão (2) (3), Gaio or Viola(2)

Bodianus speciosus (Bowdich, 1825) - Blackbar hogfish; Peixe-cão or Coa (3)

Centrolabrus caeruleus Azevedo, 1999 - Emerald wrasse; (2)

* Centrolabrus exoletus (Linnaeus, 1758) - Rock cook; Bodião(1)

$\square \quad$ Centrolabrus trutta (Lowe, 1834) - Emerald wrasse; Bodião-verde or Maracoto(2), Truta-verde or Truta-da-costa (3)

* $\square$ Coris julis (Linnaeus, 1758) - Mediterranean rainbow wrasse; Judia (1), Lambaz(2), Peixe-rei(2) (3)

Ctenolabrus rupestris (Linnaeus, 1758) - Goldsinny-wrasse; Bodião-rupestre (1)

* Labrus bergylta Ascanius, 1767 - Ballan wrasse; Bodião-reticulado(1), Bodião-vermelho(2), Trutada-costa or Truta-vermelha (3)

Labrus merula Linnaeus, 1758 - Brown wrasse; Bodião-fusco(1), (2)

* $\square$ Labrus mixtus Linnaeus, 1758 - Cuckoo wrasse; Bodião-canário (1), Peixe-rei-do-alto(2), Truta-doalto (3)

Labrus viridis Linnaeus, 1758 - Green wrasse; Bodião-torto (1)

- Lappanella fasciata (Cocco, 1833) - Pointed spotty; (2), Bodiano, Verdelhão or Verdelho(3)

* Symphodus bailloni (Valenciennes, 1839) - Baillon's wrasse; Bodião (1)

Symphodus cinereus (Bonnaterre, 1788) - Grey wrasse; Bodião-cinzento(1)

$\square \quad$ Symphodus mediterraneus (Linnaeus, 1758)-Axillary wrasse; Bodião-do-Mediterrâneo (1), Abadejo or Costureira (2), Trombetão(3)

* Symphodus melops (Linnaeus, 1758) - Corkwing; Bodião-vulgar(1), (2)

Symphodus ocellatus (Forsskål, 1775) - No common name; Bodião-de-pinta(1)

Symphodus roissali (Risso, 1810) - Five-spotted wrasse; Bodião-manchado (1)

Symphodus rostratus (Bloch, 1791) - No common name; Bodião-das-ervas (1)

Symphodus tinca (Linnaeus, 1758) - East Atlantic peacock wrasse; Bodião-pavão(1)

$\square \quad$ Thalassoma pavo (Linnaeus, 1758) - Ornate wrasse; Bodião-verde (1), Bodião, Bodeão, Rainha, Torcida or Verdugo (2), Cagão, Peixe-de-rolo or Peixe-verde (3)

$\square \quad$ Xyrichtys novacula (Linnaeus, 1758) - Pearly razorfish; Mordedor(1); Bodião-da-areia or Peixepadre (2), Papagaio (3)

Family Scaridae

$\square \quad$ Sparisoma cretense (Linnaeus, 1758) - Parrotfish; Papagaio-velho(1), Veja or Véja (2), Bodião (3)

Family Zoarcidae

Lycenchelys alba (Vaillant, 1888) - No common name; (2)

L. alba is reported off the Azores, in the PECS area (56 NM southeast of the Antialtair Seamount). Holotype collected in 1883 as Lycodes albus Vaillant, 1888, MNHN, No. 1886-0590, $21.2830^{\circ}$ W, $42.2500^{\circ}$ N. L. albus is a synonym of Lycenchelys alba.

Melanostigma atlanticum Koefoed, 1952 - Atlantic soft pout; (1)

The species M. atlanticum is regarded as being present in the Portuguese mainland EEZ based on its geographical distribution (Van der Land et al. 2001).

Pachycara thermophilum Geistdoerfer, 1994 - No common name; (2)

Pachycara saldanhai Biscoito \& Almeida, 2004 - No common name; (2) 
This species occurs off the Azores, in the PECS area (Rainbow Hydrothermal Vent Field), USNM, No.

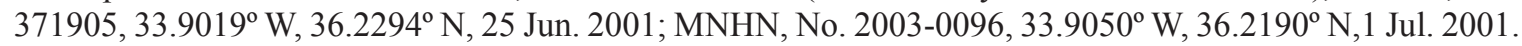

Family Chiasmodontidae

* Chiasmodon niger Johnson, 1864 - Black swallower; (1) (2), Pancinha or Sapinho-de-fundura (3)

Dysalotus alcocki MacGilchrist, 1905 - No common name; (2)

Dysalotus oligoscolus Johnson \& Cohen, 1974 - No common name; (2)

There are records of this species off the Azores, in the PECS area (13 NM NNW of the Olympus Knoll and 68 NM southwest of the Hayes Fracture Zone), MAR-ECO - HamPelFish, No. $34848750,27.8029^{\circ} \mathrm{W}, 45.6669^{\circ}$ N, 11 Jun. 1982; MAR-ECO - HamPelFish, No. 34848746, 39.6829 W, 32.9830 N, 27 Apr. 1979.

Kali indica Lloyd, 1909 - No common name; (2)

This species is recorded off the Azores, in the PECS area (96 NM northwest of the Maxwell Fracture Zone), MAR-ECO 2004, No. 6971, 29.5709 W, 48.0029 N, 25 Apr. 2004.

Kali kerberti (Weber, 1913) - No common name; (2)

$K$. kerberti is reported off the Azores, in the PECS area (13 NM northwest of the Olympus Knoll), MAR-ECO - HamPelFish, No. $34844461,27.8029^{\circ}$ W, $45.6669^{\circ}$ N, 11 Jun. 1982: identified as Kali normani (Parr, 1931). Melo (2008) re-evaluated $K$. kerberti as valid and identifies it as senior synonym of $K$. normani.

Kali macrodon (Norman, 1929) - No common name; (2) (3)

Kali macrura (Parr, 1933) - No common name; (2) (3)

Kali parri Johnson \& Cohen, 1974 - No common name; (2)

The species K. parri is reported to occur off the Azores, in the PECS area (65 NM southwest of the Hayes Fracture Zone), MAR-ECO - HamPelFish, No. 34844921, 39.6829 W, 32.9830º N, 27 Apr. 1979.

$\square \quad$ Pseudoscopelus altipinnis Parr, 1933 - No common name; (1) (2) (3)

Pseudoscopelus obtusifrons (Fowler, 1934) - No common name; (2)

Pseudoscopelus scutatus Krefft, 1971 - No common name; (2)

Family Ammodytidae

* Ammodytes tobianus Linnaeus, 1758 - Sandeel; Galeota-menor (1)

* Gymnammodytes cicerelus (Rafinesque, 1810) - Mediterranean sand eel; Galeota-da-areia (1), (2)

$\square \quad$ Gymnammodytes semisquamatus (Jourdain, 1879) - Smooth sand eel; Frachão or Galeota (1)

* Hyperoplus lanceolatus (Le Sauvage, 1824) - Great sand eel; Galeota-maior@1)

Family Trachinidae

* E Echiichthys vipera (Cuvier, 1829) - Lesser weever fish; Peixe-aranha-menor(1), Peixe-aranha(2), Aranha (3)

* Trachinus araneus Cuvier, 1829 - Spotted weever; Peixe-aranha-pontuado (1)

* $\square$ Trachinus draco Linnaeus, 1758 - Greater weever fish; Peixe-aranha-maior(1), Aranha (3)

Trachinus radiatus Cuvier, 1829 - Starry weever; Peixe-aranha-raiado(1)

Family Uranoscopidae

* $\square$ Uranoscopus scaber Linnaeus, 1758 - Stargazer; Cabeçudo (1), Papa-tabaco (3)

Family Tripterygiidae

$\square \quad$ Tripterygion delaisi Cadenat \& Blache, 1970 - Black-faced blenny; Cabrito (1), Caboz-de-trêsdorsais(2), $\mathrm{Caboz}^{(3)}$

Tripterygion tripteronotum (Risso, 1810) - No common name; Cabrito (3) 
Family Blenniidae

* Blennius ocellaris Linnaeus, 1758 - Butterfly blenny; Marachomba-borboleta (1); Caboz-ocelado(2)

* Coryphoblennius galerita (Linnaeus, 1758) - Montagu's blenny; Marachomba (1), Caboz-decrista (2), Caboz (3)

Hypleurochilus bananensis (Poll, 1959) - No common name; (1)

Hypleurochilus fissicornis (Quoy \& Gaimard, 1824) - No common name; (2)

Lipophrys canevae (Vinciguerra, 1880) - No common name; (1)

* Lipophrys pholis (Linnaeus, 1758) - Shanny; Marachomba-frade (1); Caboz-gigante (2), Caboz(3)

$\square \quad$ Lipophrys trigloides (Valenciennes, 1836) - No common name; (1), Caboz(2) (3) Microlipophrys dalmatinus (Steindachner \& Kolombatovic, 1883) - No common name; (1)

$\square \quad$ Ophioblennius atlanticus (Valenciennes, 1836) - Redlip blenny; Rói-anzóis (2), Caboz or Velha (3)

* Parablennius gattorugine (Brünnich, 1768) - Tompot blenny; Marachomba-babosa (1), (2), Caboz (3)

- Parablennius incognitus (Bath, 1968) - No common name; (1), Caboz-das-cracas (2), (3)

- Parablennius parvicornis (Valenciennes, 1836) - Rock-pool blenny; Caboz-das-poças (2), (3)

Parablennius pilicornis (Cuvier, 1829) - Ringneck blenny; (1)

Parablennius rouxi (Cocco, 1833) - No common name; (1)

- Parablennius ruber (Valenciennes, 1836) - Portuguese blenny; Caboz-português (1) (2), Cabozlusitano (2), (3) Caboz

$\square \quad$ Parablennius sanguinolentus (Pallas, 1814) - Rusty blenny; (1), Caboz-das-poças (2), Caboz(3)

Parablennius tentacularis (Brünnich, 1768) - Tentacled blenny; (1)

This species is regarded as being present off mainland Portugal, based on its geographical distribution (Eschmeyer 2013) and because the presence of $P$. tentacularis has been reported off the Portuguese coast (Gordo \& Cabral 2001).

* Salaria pavo (Risso, 1810) - Peacock blenny; Marachomba-pavão(1), (3)

Family Clinidae

* Clinitrachus argentatus (Risso, 1810) - Cline; Peixe-macaco (1)

Family Labrisomidae

$\square \quad$ Labrisomus nuchipinnis (Quoy \& Gaimard, 1824) - Hairy blenny; (3)

Family Gobiesocidae

Apletodon dentatus (Facciolà, 1887) - Small-headed clingfish; Pegador(1)

Apletodon incognitus Hofrichter \& Patzner, 1997 - No common name; (1), Peixe-ventosa-dosouriços (2), (3)

$\diamond \quad$ Apletodon pellegrini (Chabanaud, 1925) - Chubby clingfish; Peixe-ventosa (2), Sugador(3)

- Diplecogaster bimaculata (Bonnaterre, 1788) - Two-spotted clingfish; Pegador(1), (3)

Diplecogaster bimaculata pectoralis Briggs, 1955 - No common name; Peixe-ventosa(2)

* Lepadogaster candolii Risso, 1810 - Connemarra clingfish; (1) (3)

Almada et al. (2008) indicated the need to remove the species L. candollei from the genus Lepadogaster. Canestrini (1864) had previously suggested placing L. candollei in a different genus (Mirbelia), distinct from Lepadogaster. In turn Briggs (1955), in his detailed revision, although placing L. candollei in Lepadogaster noted that: " $L$. candollei is well separated from the other three forms [of Lepadogaster] which are very closely related to each other".

* $\square$ Lepadogaster lepadogaster (Bonnaterre, 1788) - Shore clingfish; Sugador(1) (3), Chupa-sangue (3) The species Lepadogaster zebrina Lowe, 1839 is a synonym of L. lepadogaster, according to Almada et al. (2008). All records of L. zebrine should be considered as L. lepadogaster. 
- Lepadogaster purpurea (Bonnaterre, 1788) - Cornish sucker; Sugador(1), (2) (3)

This species is recorded off the Azores, in the PECS area (157 NM southwest of the Georgiy Zima Seamount), VLIZ, Tisbe No. $318571,20.0^{\circ} \mathrm{W}, 40.0^{\circ} \mathrm{N}$.

Opeatogenys gracilis (Canestrini, 1864) - No common name; (1)

\section{Family Callionymidae}

* Callionymus lyra Linnaeus, 1758 - Common dragonet; Peixe-pau-lira (1), (2)

* $\square$ Callionymus maculatus Rafinesque-Schmaltz, 1810 - Spotted dragonet; Peixe-pau-malhado(1)

Callionymus pusillus Delaroche, 1809 - No common name; Peixe-pau-rabudo(1)

Callionymus reticulatus Valenciennes, 1837 - Reticulate dragonet; Peixe-pau-listado (1), (2)

* Callionymus risso Lesueur, 1814 - No common name; Peixe-pau-pintado (1)

- Protogrammus sousai (Maul, 1972) - No common name; (2)

This species is reported off the Azores, in the PECS area (Great Meteor Tablemount), SDSC, No. 23042, $28.4833^{\circ} \mathrm{W}, 29.8258^{\circ} \mathrm{N}$; SDSC, No. 23041, $28.4966^{\circ} \mathrm{W}, 29.8366^{\circ} \mathrm{N}$; SDSC, No. $23043,28.4000^{\circ} \mathrm{W}$, $30.0183^{\circ} \mathrm{N}$.

* $\square$ Synchiropus phaeton (Günther, 1861) - No common name; Peixe-pau-rosa (1) (2), (2) (3)

Family Draconettidae

- Centrodraco acanthopoma (Regan, 1904) - No common name; (1) (2) (3)

There are records of $C$. acanthopoma off the Azores, in the PECS area (Great Meteor Tablemount), SDSC, No. 23046, $28.5249^{\circ} \mathrm{W}, 30.0499^{\circ} \mathrm{N}, 25$ Jul. 1967; SDSC, No. 9286, $28.6667^{\circ} \mathrm{W}, 30.0499^{\circ} \mathrm{N}, 25$ Jul. 1967; SDSC, No. 23045, 28.6667 W, 30.0499 ${ }^{\circ}$ N, 25 Jul. 1967.

\section{Family Gobiidae}

* Aphia minuta (Risso, 1810) - Transparent goby; Caboz-transparente (1)

Buenia jeffreysii (Günther, 1867) - Jeffrey's goby; (1)

- Chromogobius britoi Van Tassell, 2001 - Brito's goby; (1) (3)

$\square \quad$ Crystallogobius linearis (Düben, 1845) - Crystal goby; (1) (3)

$\square \quad$ Deltentosteus collonianus (Risso, 1820) - No common name; (1)

* Deltentosteus quadrimaculatus (Valenciennes, 1837) - Four-spotted goby; Caboz-de-quatromanchas (1)

$\square \quad$ Gnatholepis thompsoni Jordan, 1904 - No common name; (1) (3)

Gobius ater Bellotti, 1888 - Bellotti's goby; (1)

* Gobius auratus Risso, 1810 - Golden goby; Caboz-dourado or Caboz-das-pedras (1), (3)

Gobius bucchichi Steindachner, 1870 - Bucchich's goby; (1)

* Gobius cobitis Pallas, 1814 - Giant goby; Caboz-cabeçudo(1)

Gobius cruentatus Gmelin, 1789 - Red-mouthed goby; Caboz-boca-vermelha(1)

$\square \quad$ Gobius gasteveni Miller, 1974 - Steven's goby; (1), Caboz-de-escama (3)

* Gobius niger Linnaeus, 1758 - Black goby; Caboz-negro(1)

* Gobius paganellus Linnaeus, 1758 - Rock Goby; Caboz-da-rocha(1); Bochecha or Joana(2), Caboz(2) (3), Velha (3)

$\square \quad$ Gobius roulei de Buen, 1928 - Roule's goby; (1) (3)

Gobius xanthocephalus Heymer \& Zander, 1992 - No common name; (1)

* Gobiusculus flavenscens (Fabricius, 1779) - Two-spotted goby; Caboz or Alcaboz(1)

Lebetus guilleti (Le Danois, 1913) - Guillet's goby; (1) (3)

Lebetus scorpioides (Collet, 1874) - Diminutive goby; (1)

* Lesueurigobius friesii (Malm, 1874) - Fries's goby; Caboz-de-escama-grande (1)

$\square \quad$ Lesueurigobius heterofasciatus Maul, 1971 - Striped goby; (3) 
Lesueurigobius sanzi (de Buen, 1918) - Sanzo's goby; Caboz-de-bandeira(1)

Lesueurigobius suerii (Risso, 1810) - Lesueur's goby; (1) (2) (3)

$\square \quad$ Mauligobius maderensis (Valenciennes, 1837) - No common name; (2), Caboz-de-escama (3)

Pomatoschistus knerii (Steindachner, 1861) - Kner's goby; (1)

Pomatoschistus lozanoi (de Buen, 1923) - Lozano's goby; (1)

Pomatoschistus marmoratus (Risso, 1810) - Marbled goby; (1)

Pomatoschistus microps (Kroyer, 1838) - Common goby; Caboz(1)

* Pomatoschistus minutus (Pallas, 1770) - Sand goby; Caboz-da-areia (1)

- Pomatoschistus pictus (Malm, 1865) - Painted goby; (1), Góbio-da-areia (2), (3)

$\square \quad$ Thorogobius ephippiatus (Lowe, 1839) - Leopard-spotted goby; (1), Bochecha-pintada (2), Caboz or Caboz-de-escama (3)

Vanneaugobius canariensis Van Tassel, Miller \& Brito, 1988 - No common name; (3)

Family Luvaridae

Luvarus imperialis Rafinesque, 1810 - Luvar; (1), Boquinho or Peixe-sol(2), Boquinho(3)

Family Acanthuridae

Acanthurus monroviae Steindachner, 1876 - Monrovia doctorfish; Unha (1)

Family Scombrolabracidae

$* \diamond \square$ Scombrolabrax heterolepis Roule, 1921 - Longfin escolar; (1) (2) (3)

New record for Portuguese mainland waters; the first specimen of S. heterolepis (Fig. 4) was caught on the $15^{\text {th }}$ of November 2008 ( $300 \mathrm{~mm}$ TL and $375.63 \mathrm{~g}$ ), using a bottom trawl $\left(39^{\circ} 59.257^{\prime} \mathrm{N}, 10^{\circ} 3.025^{\prime} \mathrm{W}\right)$ and other specimens were collected in commercial fisheries in 2008 and 2009. Bold Systems Sample ID MLFPI62, available on the Barcode of Life Data Systems (BOLD; www.barcodinglife.org, under the project titled "Fish of Portugal and Italy [MLFPI]").

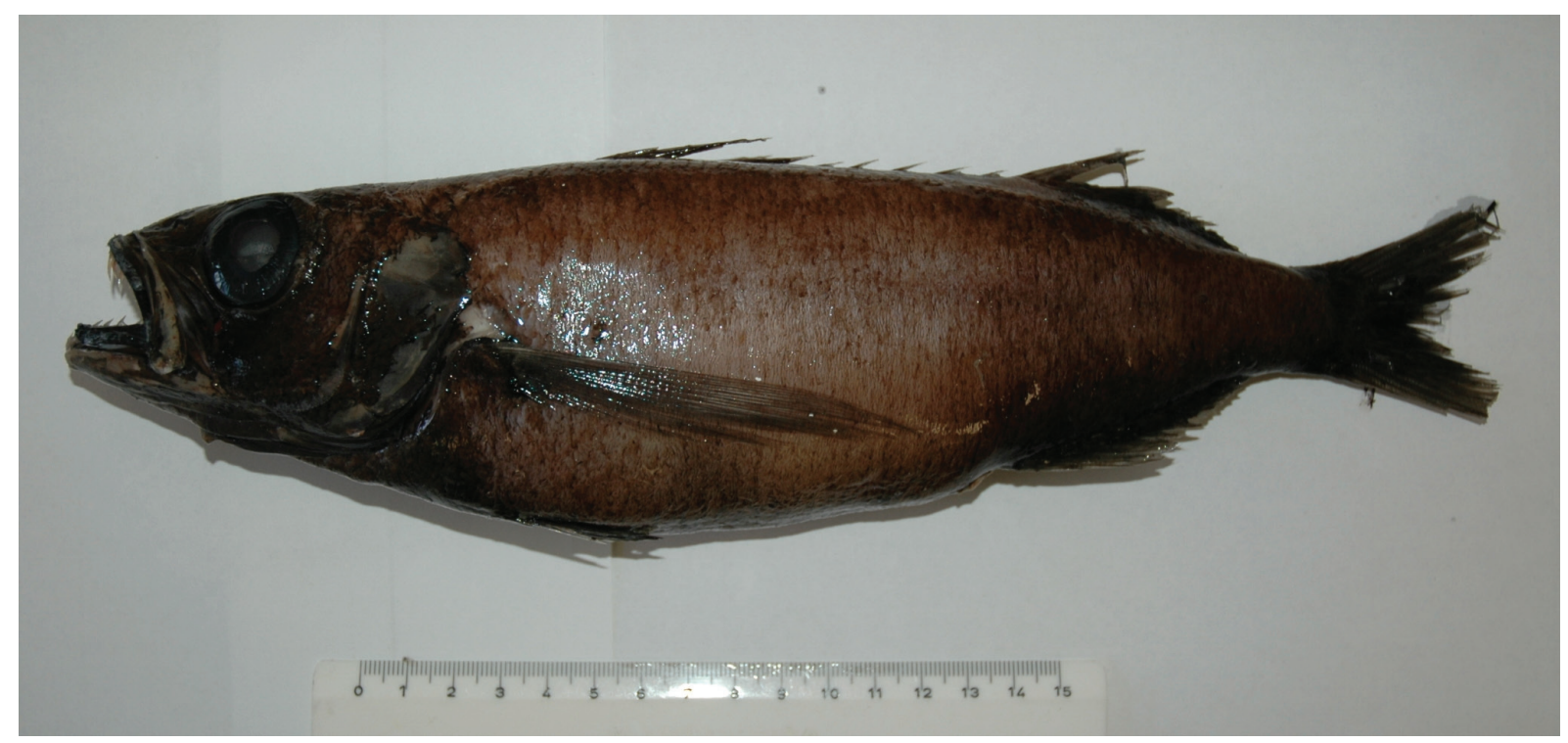

Fig. 4. Scombrolabrax heterolepis Roule, 1921. 
Family Sphyraenidae

Sphyraena barracuda (Edwards, 1771) - Great barracuda; Bicuda-gigante (3)

* Sphyraena sphyraena (Linnaeus, 1758) - European barracuda; Bicuda (1), (2)

$\square \quad$ Sphyraena viridensis Cuvier, 1829 - Yellowmouth barracuda; (1), Bicuda(2) (3)

According to Wirtz (1998), the records of S. sphyraena, reported from Madeira, are likely to be confused with the common S. viridensis.

\section{Family Gempylidae}

$\square \quad$ Diplospinus multistriatus Maul, 1948 - Striped escolar; (2) (3)

$\square \quad$ Gempylus serpens Cuvier, 1829 - Snake mackerel; Escolar(2), Coelho-de-natura (3)

$* \diamond \square$ Lepidocybium flavobrunneum (Smith, 1843) - Escolar; Escolar-preto(1), Escolar(2), Escolar-denatura (3)

$\square \quad$ Nealotus tripes Johnson, 1865 - Black snake mackerel; (2), Peixe-coelho-de-natura(3)

* $\square$ Nesiarchus nasutus Johnson, 1862 - Black gemfish; Geribé (1), Escolar(2), Coelho-de-natura (1) (3)

$\square \quad$ Promethichthys prometheus (Cuvier, 1832) - Roudi escolar; Escolar-branco(1), Coelho-do-alto(2), Peixe-coelho(2) (3)

Ruvettus pretiosus Cocco, 1833 - Oilfish; Escolar(1) (2) (3)

\section{Family Trichiuridae}

* Aphanopus carbo Lowe, 1839 - Black scabbardfish; Peixe-espada-preto (1) (2), Espada or Espadapreto (3)

Aphanopus intermedius Parin, 1983 - Intermediate scabbardfish; (2) (3)

Benthodesmus elongatus (Clarke, 1879) - Elongate frostfish; Espada-de-má-água (1), Espadabranca-de-natura (3)

* Benthodesmus simonyi (Steindachner, 1891) - Simony’s frostfish; (1); Peixe-espada (2), (3)

* Lepidopus caudatus (Euphrasen, 1788) - Silver scabbard fish; Peixe-espada-branco(1) (2), Talabarte(2), Espada-branca (3)

* Trichiurus lepturus Linnaeus, 1758 - Largehead hairtail; Lírio (1)

\section{Family Scombridae}

$\diamond \quad$ Acanthocybium solandri (Cuvier, 1832) - Wahoo; Cavala-da-Índia(1) (2) (3)

* $\square$ Auxis rochei rochei (Risso, 1810) - Bullet tuna; Judeu (1) (2) (3)

* $\square$ Auxis thazard thazard (Lacepède, 1800) - Frigate tuna; Judeu-liso(1), Chapouto (3)

Euthynnus alletteratus (Rafinesque, 1810) - Little tunny; Merma (1)

$\square \quad$ Katsuwonus pelamis (Linnaeus, 1758) - Skipjack tuna; Gaiado (1) (2) (3), Bonito (1) (2)

* $\square$ Sarda sarda (Bloch, 1793) - Atlantic bonito; Sarrajão (1) (3), Serra (1) (2), Cerda or Serralhão (3)

* $\square$ Scomber colias Gmelin, 1789 - Atlantic chub mackerel; Cavala (1) (2) (3)

* $\square$ Scomber scombrus Linnaeus, 1758 - Atlantic mackerel; Sarda (1), Cavala (2) (3)

- Thunnus alalunga (Bonnaterre, 1788) - Albacore; Atum-voador(1) (2) (3), Voador(1) (2) (3), Avoador(3)

$\square \quad$ Thunnus albacares (Bonnaterre, 1788) - Yellowfin tuna; Atum-albacora (1) (3), Albacora (1) (2), Alvacor or Galha-à-ré(2)

Thunnus atlanticus (Lesson, 1831) - Blackfin tuna; Albacorinha, (2)

The species T. atlanticus occurs off the Azores, in the PECS area (41 NM west of the Ocenographer Fracture Zone), MCZ, No. $75623,35.8133^{\circ} \mathrm{W}, 35.0750^{\circ} \mathrm{N}, 26$ Aug. 1984.

$\square \quad$ Thunnus obesus (Lowe, 1839) - Bigeye tuna; Atum-patudo (1) (3), Patudo (1) (2) (3), Albacora, Alvacor or Alvacora (2) 
Thunnus thynnus (Linnaeus, 1758) - Atlantic bluefin tuna; Atum-rabilho (1), Albacora, Alvacor, Rabão or Rabilo(2), Atum-rabil or Rabilho (3)

\section{Family Xiphiidae}

* $\square$ Xiphias gladius Linnaeus, 1758 - Swordfish; Espadarte (1) (2), Peixe-agulha (1) (3), Agulhão(2)

Family Istiophoridae

Istiophorus platypterus (Shaw \& Nodder, 1792) - Sailfish; Veleiro-do-Atlântico (1), (2) (3)

Istiophorus albicans (Latreille, 1804) is a synonym of I. platypterus according to Collette et al. (2006).

Kajikia albida (Poey, 1860) - Atlantic white marlin; Espadim-branco-do-Atlântico(1), Espadimbranco or Marlin-branco(2), Peto(3)

Makaira nigricans Lacepède, 1802 - Blue marlin; Espadim-azul-do-Atlântico (1), Espadim-azul (1) (2), (3)

Tetrapturus belone Rafinesque, 1810 - Mediterranean spearfish; Espadim-do-Mediterrâneo, Peto (3) Tetrapturus georgii Lowe, 1841 - Roundscale spearfish; Espadim-peto(1), (2), Peito(3)

$\diamond \quad$ Tetrapturus pfluegeri Robins \& de Sylva, 1963 - Longbill spearfish; Espadim-bicudo(1), (2)

\section{Familly Centrolophidae}

* $\square$ Centrolophus niger (Gmelin, 1789) - Rudderfish; Liro-preto (1), (2), Liro (3)

Hyperoglyphe perciformis (Mitchill, 1818) - Barrelfish; Liro (1) (2)

- Schedophilus maculatus Günther, 1860 - Pelagic butterfish; (2) (3)

- Schedophilus medusophagus (Cocco, 1839) - Cornish blackfish; Liro-mole (1), (2) (3)

* $\square$ Schedophilus ovalis (Cuvier, 1833) - Imperial blackfish; Liro-imperial (1), Choupa (2), Lírio or Liro (3)

Family Nomeidae

* Cubiceps gracilis (Lowe, 1843) - Driftfish; Tirone (1), (2) (3)

Nomeus gronovii (Gmelin, 1789) - Man-of-war fish; Pequeno-argonauta(2)

Psenes cyanophrys Valenciennes, 1833 - Freckled driftfish; (3)

Psenes maculatus Lütken, 1880 - Silver driftfish; (2)

\section{Family Tetragonuridae}

Tetragonurus atlanticus Lowe, 1839 - Bigeye squaretail; (1) (2), Escolar-de-natura(3)

$\square \quad$ Tetragonurus cuvieri Risso, 1810 - Smalleye squaretail, (1) (2), Escolar-de-natura (3)

Family Stromateidae

* Stromateus fiatola Linnaeus, 1758 - Blue butterfish; Pampo-godinho (1), (2) (3)

Family Caproidae

* Antigonia capros Lowe, 1843 - Deepbody boarfish, Periquito (1), (2) (3)

$* \square$ Capros aper (Linnaeus, 1758) - Boarfish; Pimpim (1) (2), Peixe-pau (2), Tem-te-em-pé(3)

Order Pleuronectiformes

Family Citharidae

* Citharus linguatula (Linnaeus, 1758) - Atlantic spotted flounder; Carta-de-bico(1), (3) 
Family Scophthalmidae

* $\square$ Lepidorhombus boscii (Risso, 1810) - Fourspotted megrim; Areeiro-de-quatro-manchas (1), (2) (3) The species L. boscii is recorded off Madeira (Josephine Bank) and to the northeast of the Azores (157 NM southwest of the Georgiy Zima Seamount), both outside their respective EEZ's. MNHN, No. 1956-0007, $20.0^{\circ} \mathrm{W}, 40.0^{\circ} \mathrm{N}$; MNHN, No. $1956-0008,20.0^{\circ} \mathrm{W}, 40.0^{\circ} \mathrm{N}$, both specimens captured in June 1955; FISH 1556599 (Shcherbachev et al. 1985).

* Lepidorhombus whiffiagonis (Walbaum, 1792) - Megrim; Areeiro(1) (2), Solha-da-fundura (2)

* Scophthalmus maximus (Linnaeus, 1758) - Turbot; Pregado(1), (3)

* Scophthalmus rhombus (Linnaeus, 1758) - Brill; Rodovalho(1)

* Zeugopterus punctatus (Bloch, 1787) - Topknot; Rodovalho-bruxa(1)

* Zeugopterus regius (Bonnaterre, 1788) - Eckström's topknot; Bruxa(1)

Family Paralichthyidae

Syacium papillosum (Linnaeus, 1758) - Dusky flounder; (2)

This species is recorded off the Azores, in the PECS area (82 NM west of the Great Meteor Tablemount), ROM, No. 23911, 30.2500 W, 30.2916º N, 29 Jan. 1966.

Family Pleuronectidae

Microstomus kitt (Walbaum, 1792) - Lemon sole; Solha-limão(1)

* Platichthys flesus (Linnaeus, 1758) - European flounder; Solha-das-pedras or Patruça (1)

* Pleuronectes platessa Linnaeus, 1758 - European plaice; Solha(1), (2)

Family Bothidae

* $\square$ Arnoglossus imperialis (Rafinesque, 1810) - Imperial scaldfish; Carta-imperial (1), (2) (3)

* Arnoglossus laterna (Walbaum, 1792) - Scald fish; Carta-do-Mediterrâneo (1)

* Arnoglossus rueppelii (Cocco, 1844) - Ruppell's scaldback; Carta-estreita (1), (2) (3)

* Arnoglossus thori Kyle, 1913 - Thor's scaldfish; Carta-pontuada (1), (3)

* B Bothus podas (Delaroche, 1809) - Wide-eyed flounder; Carta-de-olhos-grandes (1) (2), Solha (2) (3)

Chascanopsetta lugubris Alcock, 1894 - Pelican flounder; Carta-pelicano (1)

Monolene microstoma Cadenat, 1937 - Smallmouth moonflounder; Carta-de-boca-pequena, (3)

This species is reported from Madeira, in the PECS area (Josephine Bank), based on a single observation,

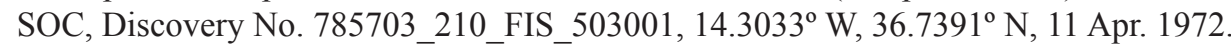

Family Soleidae

* Bathysolea profundicola (Vaillant, 1888) - Deepwater sole; Linguado-da-fundura (1)

Buglossidium luteum (Risso, 1810) - Solenette; Língua-de-gato (1), (2)

The species B. luteum has been recorded from the northeast of the Azores, in the PECS area (157 NM southwest of the Georgiy Zima Seamount), MNHN, No. 1959-0604, 20.0 $0^{\circ} \mathrm{W}, 40.0^{\circ} \mathrm{N}$.

* Dicologlossa cuneata (Moreau, 1881) - Wedge sole; Língua (1), (3)

* Dicologlossa hexophthalma (Bennett, 1831) - Ocellated wedge sole; Linguado-de-olhos (1), (3)

* Microchirus azevia (de Brito Capello, 1867) - Bastard sole; Azevia (1)

* Microchirus boscanion (Chabanaud, 1926) - Lusitanian sole; Azevia-marginada (1), (3)

* $\square$ Microchirus ocellatus (Linnaeus, 1758) - Foureyed sole; Azevia-de-malhas (1), Solha-linguado(3)

* Microchirus variegatus (Donovan, 1808) - Thickback sole; Azevia-raiada or Raposo (1), (3)

Microchirus wittei Chabanaud, 1950 - Banded sole; Azevia-raiada-africana (3)

* Monochirus hispidus Rafinesque, 1814 - Whiskered sole; Cascarra(1)

* Pegusa impar (Bennett, 1831) - Adriatic sole; (1) 
* Pegusa lascaris (Risso, 1810) - Sand sole; Linguado-da-areia (1), (2) (3)

* Solea senegalensis Kaup, 1858 - Senegalese sole; Linguado-branco(1), (3)

* Solea solea (Linnaeus, 1758) - Common sole; Linguado-legítimo(1), (3)

* Synaptura lusitanica lusitanica de Brito Capello, 1868 - Portuguese sole; Língua-de-vaca or Linguado-português (1)

Synapturichthys kleinii (Risso, 1827) - Klein's sole; (1)

\section{Family Cynoglossidae}

․ Symphurus insularis Munroe, Brito \& Hernández, 2000 - No common name; (2) (3)

* $\square$ Symphurus nigrescens Rafinesque, 1810 - Tonguesole; Língua-avessa (1), Língua(2) Symphurus reticulatus Munroe, 1990 - No common name; (3)

Order Tetraodontiformes Family Balistidae

* $\square$ Balistes capriscus Gmelin, 1789 - Grey triggerfish; Cangulo-cinzento (1), Peixe-porco (2) (3), Peixeburro (3)

Balistes punctatus Gmelin, 1789 - Bluespotted triggerfish; Cangulo-pintado, (3)

This seems a doubtful record for Madeira, which needs confirmation (Wirtz et al. 2008).

Balistes vetula Linnaeus, 1758 - Queen triggerfish; Peixe-porco (2)

$\diamond \quad$ Canthidermis maculata (Bloch, 1786) - Ocean triggerfish; Peixe-porco(2)

$\square \quad$ Canthidermis sufflamen (Mitchill, 1815) - No common name; (2) (3)

The species C. sufflamen occurs off the Azores, in the PECS area (82 NM west of the Great Meteor Tablemount), ROM, No. 23921, 30.2500 ${ }^{\circ}$ W, $30.2916^{\circ}$ N, 29 Jan. 1966.

Family Monacanthidae

$\diamond \square$ Aluterus monoceros (Linnaeus, 1758) - Unicorn leatherjacket filefish; Peixe-porco (2), (3)

$\diamond \square$ Aluterus scriptus (Osbeck, 1765) - Scribbled leatherjacket filefish; (2) (3)

$\diamond \square$ Stephanolepis hispidus (Linnaeus, 1766) - Planehead filefish; Peixe-porco(2), Peixe-porcogalhudo (3)

Stephanolepis setifer (Bennett, 1831) - Pygmy filefish; (3)

Family Ostraciidae

Acanthostracion notacanthus (Bleeker, 1863) - Island boxfish; (2)

Acanthostracion quadricornis (Linnaeus, 1758) - Scrawled cowfish; (2)

This species is recorded off the Azores, in the PECS area (82 NM west of the Great Meteor Tablemount), ROM, No. 23915, 30.2500 ${ }^{\circ}$ W, 30.2916 N, 29 Jan. 1966.

\section{Family Tetraodontidae}

Canthigaster capistrata (Lowe, 1839) - Sharpnose puffer; (2), Porquinho or Sapo (3)

$\diamond \quad$ Canthigaster rostrata (Bloch, 1786) - Sharpnose puffer; Peixe-balão or Porquinho(1) (2), Sapinho(2) All Canthigaster specimens reported so far from Madeira belong to Canthigaster capistrata (Moura \& Castro 2002). There is a doubtful record from off the Portuguese mainland that requires confirmation.

Ephippion guttifer (Bennett, 1831) - Prickly puffer; (1) (3)

$\square \quad$ Lagocephalus laevigatus (Linnaeus, 1758) - Smooth puffer; Baiacu-verde(1), (3)

* Lagocephalus lagocephalus lagocephalus (Linnaeus, 1758) - Oceanic puffer; (1), Peixe-balão(2), Sapo(3) 
$\square \quad$ Sphoeroides marmoratus (Lowe, 1838) - Guinean puffer; (1), Peixe-balão, Sopapo or Sapo(2), Sapinho(3)

The records for Sphoeroides spengleri (Bloch, 1785) should be replaced by S. marmoratus in the eastern Atlantic (P. Wirtz, pers. comm. Oct. 2004, in FishBase, Froese \& Pauly 2012).

* Sphoeroides pachygaster (Müller \& Troschel, 1848) - Blunthead puffer; Peixe-bola (1), Peixe-balão or Sapo-do-alto (2), (3)

Family Diodontidae

$\diamond \square$ Chilomycterus atringa (Linnaeus, 1758) - Porcupine fish; Peixe-porco (1), (2), Sapo (3)

$\diamond \square$ Chilomycterus reticulatus (Linnaeus, 1758) - Spotfin burrfish; Peixe-porco (1), (2), Sapo (3)

Chilomycterus schoepfii (Walbaum, 1792) - No common name; (2)

This species is recorded off the Azores, in the PECS area (82 NM west of the Great Meteor Tablemount), ROM, No. 23914, 30.2500 W, $30.2916^{\circ} \mathrm{N}, 29$ Jan. 1966.

* Chilomycterus spinosus spinosus (Linnaeus, 1758) - No common name; (1)

Diodon eydouxii Brisout de Barneville, 1846 - Pelagic porcupinefish; Peixe-ouriço(2)

$\diamond$ Diodon holocanthus Linnaeus, 1758 - Longspined porcupinefish; Peixe-ouriço-de-crista, (2)

$* \diamond \square$ Diodon hystrix Linnaeus, 1758 - Spot-fin porcupinefish; Peixe-ouriço(1) (2), Sapo-grande(1) (3), Peixe-balão-espinhoso (2), Sapo or Sapo-de-espinhos (3)

Family Molidae

$\square \quad$ Masturus lanceolatus (Liénard, 1840) - Sharptail mola; Peixe-lua-rabudo(1) (2), Peixe-lua (2) (3), Peixe-porco (3)

* Mola mola (Linnaeus, 1758) - Ocean sunfish; Peixe-lua(1) (2) (3)

$* \diamond \square$ Ranzania laevis (Pennant, 1776) - Slender sunfish; Peixe-lua-comprido (1) (2), Peixe-lua (2), (3)

\section{Discussion}

The present checklist compiles for the first time an annotated list of fish species occurring both in the Portuguese EEZ and territorial waters, and in the area corresponding to the proposed extension of the Portuguese continental shelf (PECS). Based solely on updated information on species occurrences, this new checklist results in an addition of over 200 species to the Portuguese marine ichthyofauna, which corresponds to a relevant increase of about $28 \%$ compared to earlier and current compilations (Froese \& Pauly 2012; Magalhães \& Rogado 1993). To this primary list we add 130 new species records from the area corresponding to the PECS. Therefore, the grand total of 1191 marine fishes included here represent approximately $88 \%$ of the 1349 species listed for Europe in the European Register of Marine Species (Costello et al. 2006), although the surveyed areas do not match completely.

The great increase in species numbers in the current checklist can be justified in a number of ways. Part of this increase is a consequence of new records for deep-sea fishes obtained from recent deepsea surveys. We also recovered a number of old records and found new relatively recent records in the literature that had not yet been included in databases or that had not been considered in previous checklists (e.g., Vieira et al. 2012; Menezes et al. 2012).

There is growing scientific evidence that, as a result of global warming, the traditional pattern of species distribution seems to have altered in the last decades (since about the mid-1980s). In general, a warmer oceanic temperature would result in poleward advances of species with an affinity to warm water (Lusitanian) and the retreat of species with an affinity to cooler water (Boreal) (Brander et al. 2003; Southward et al. 2005; Stefansdottir et al. 2010). 
From our data we could not infer any global trend of this kind, since most of the records in the checklist lack historical occurrence data. However, it is possible to identify some poleward expansions towards the Iberian coast and the Azores among tropical Atlantic fishes, such as occurred, for example, with Fistularia petimba (Azevedo et al. 2004; Bañon \& Sande 2008), Zenion hololepis (Martins et al. 2012) and Acanthurus monroviae (Horta e Costa \& Gonçalves 2013). Conversely, there is evidence of the contraction of southern range limits of boreal species towards higher latitudes, and the decrease of their records, as for example observed for Salmo salar and Cyclopterus lumpus.

Quéro et al. (1998) investigated the alteration of the northward range extension of fish species associated with the warming of European Atlantic waters since 1963. The upper slope species have made regular northward range extensions off southern Portugal to northwestern Ireland, more and less rapidly, taking about thirty years for Cyttopsis roseus and Zenopsis conchifer and only six years for Sphoeroides pachygaster. Another example of these alterations is the substantial increase in the abundance of a subtropical species, the boarfish Capros aper, that correlates with the temperature increase on the northeast Atlantic continental shelf (Blanchard et al. 2005; Coad et al. 2012).

Owing to the fact that marine ecosystems are influenced by many factors, the interpretation of alterations in species ranges may not be simple. Some of these factors are strongly correlated, as for biogeochemical cycles $\left(\mathrm{CO}_{2}\right)$ and the eduction of the $\mathrm{pH}$ of surface seawater (acidification); primary production, nutrient availability and foodwebs; overfishing, recruitment and phenological relationships. The establishment of invasive species may also have a far-reaching effect. Those factors may provide a growing contribution to the threatened biodiversity at a global scale because of the cumulative impact of different variables. The NRIC (National and Regional Implementation Committees / Census of Marine Life) reported overfishing, habitat loss, and pollution (contamination by xenobiotics and eutrophication), to be the greatest threats to marine biodiversity, followed by alien species and the impacts of warming due to climate change (Costello et al. 2010).

In the present work we chose to apply a large spatial scale to our biogeographical analysis. Globally, the biogeographical composition analysis revealed that the Atlantic group is the largest, with almost the $46.01 \%$ of the total species represented. The Lusitanian $(21.49 \%)$ and the African (5.96\%) groups are also important. Six other groups are minor, including the Boreal group, the Mediterranean group, the Macaronesian group, the Atlantic/African group, the Mediterranean/African group and the Arctic group.

By providing a biogeographic classification of the species in the current checklist, we aim at contributing to the understanding of the distribution of species and habitats for the purposes of scientific research, conservation and management, and ultimately for policy definition. This is particularly relevant given that the effective application of the proposed extension of the EEZ may result in one of the largest EEZ of the world, covering a major section of the northeast Atlantic. Scientifically, this biogeographic classification can provide a basis for hypotheses and further scientific studies on the origin and evolution of deep-sea fauna assemblages and the linkage between species communities and open-ocean and deepsea environments. From a policy perspective, such a classification is a necessary component, when considering area-based management options, such as marine protected areas, particularly when assessing representativity of a potential network (Vierros et al. 2009).

\section{Acknowledgements}

We are grateful to Pedro Victor and to the fishermen from Peniche and Sesimbra, who provided some fish specimens, in particular the species Bajacalifornia megalops, Salmo salar, Setarches guentheri and Scombrolabrax heterolepis. We thank Alvaro Pesqueira (skipper and fisher) and Miguel Caetano for providing the specimens of Fistularia petimba. We wish to acknowledge the constructive comments of the scientific editor of this paper and two anonymous reviewers, which helped improve our manuscript. 
This work was supported by FEDER through POFC-COMPETE and by national funds from "Fundação para a Ciência e a Tecnologia (FCT)" in the scope of the grants FCOMP-01-0124-FEDER-010596 and PEst-C/BIA/UI4050/2011. M. Landi's work was supported by the FCT fellowship SFRH/ $\mathrm{BPD} / 45246 / 2008$.

\section{References}

Albuquerque R.M. 1954-1956. Peixes de Portugal e Ilhas Adjacentes (Chaves para a sua determinação). Portugaliae Acta Biológica, Lisboa.

Almaça C. 1998. On the sturgeon, Acipenser sturio, in the Portuguese rivers and seas. Folia Zoologica 37: 183-191.

Almada F., Henriques M., Levy A., Pereira A., Robalo J. \& Almada V.C. 2008. Reclassification of Lepadogaster candollei based on molecular and meristic evidence with a redefinition of the genus Lepadogaster. Molecular Phylogenetics and Evolution 46: 1151-1156. http://dx.doi.org/10.1016/j.ympev.2007.05.021

Andriashev A.P. 1998. A review of recent studies of Southern Ocean Liparidae (Teleostei: Scorpaeniformes). Cybium, 22 (3): 255-266.

Andriashev A.P. 2003. Liparid fishes (Liparidae, Scorpaeniformes) of the Southern Ocean and adjacent waters. Results of Russian Antarctic Expeditions 9. Explorations of the Fauna of the Seas, 53 (61): $1-477$.

Arruda L.M. 1997. Checklist of the marine fishes of the Azores. Arquivos do Museu Bocage, Nova Série 3 (2): 3-164.

Aschliman N.C., Nishida M., Miya M., Inoue J.G., Rosana K.M. \& Naylor G.J.P. 2012. Body plan convergence in the evolution of skates and rays (Chondrichthyes: Batoidea). Molecular Phylogenetics and Evolution 63 (1): 28-42. http://dx.doi.org/10.1016/j.ympev.2011.12.012

Azevedo J.M.N., Raposeiro P.M. \& Rodrigues L. 2004. First records of Fistularia petimba and Diodon eydouxii for the Azores, with notes on the occurrence of three additional species. Journal of Fish Biology 65 (4): 1180-1184. http://dx.doi.org/10.1111/j.0022-1112.2004.00523.x

Bañón R. \& Sande C. 2008. First record of the red cornetfish Fistularia petimba (Syngnathiformes: Fistularidae) in Galician waters: a northernmost occurrence in the eastern Atlantic. Journal of Applied Ichthyology 24 (1): 106-107. http://dx.doi.org/10.1111/j.1439-0426.2007.00918.x

Bañón R., Arronte J.C., Vázquez-Dorado S., Del Río J.L. \& Carlos A. 2012. DNA barcoding of the genus Lepidion (Gadiformes: Moridae) with recognition of Lepidion eques as a junior synonym of Lepidion lepidion. Molecular Ecology Resources 13: 189-199. http://dx.doi.org/10.1111/1755-0998.12045

Bauchot M.-L. \& Saldanha L. 1986. Congridae (including Heterocongridae). In: Whitehead P.J.P., Bauchot M.-L., Hureau J.-C., Nielsen J. \& Tortonese E. (eds) Fishes of the North-Eastern Atlantic and the Mediterranean 2: 567-574. UNESCO, Paris.

Blanchard F. \& Vandermeirsch F. 2005. Warming and exponential abundance increase of the subtropical fish Capros aper in the Bay of Biscay (1973-2002). Comptes Rendus Biologies 328: 505-509. http:// dx.doi.org/10.1016/j.crvi.2004.12.006

Bonnet C. 1850. Mémoire sur le Royaume de l'Algarve. Academia Real das Sciencias, 2a Série, 2, Lisboa.

Brander K., Blom G., Borges M.F., Erzini K., Henderson G., MacKenzie B.R., Mendes H., Ribeiro J., Santos A.M.P. \& Toresen R. 2003. Changes in fish distribution in the eastern North Atlantic: Are we 
seeing a coherent response to changing temperature? International Council for the Exploration of the Sea - Marine Science Symposium 219: 261-270.

Brauer A. 1906. Wissenschaftliche Ergebnisse der Deutschen Tiefsee-Expedition auf dem Dampfer „Valdivia“ 1898-1899. Jena, Naumburg.

Briggs J.C. 1955. A Monograph of the Clingfish (Order Xenopterygii). Stanford Ichthyological Bulletin 6: 33-39.

Canestrini G. 1864. Studi sui Lepadogaster del Mediterraneo. Archivio per la Zoologia, L'Anatomia e la Fisiologia 3: 177-196.

Capello F.B. 1867a. Catálogo dos peixes de Portugal que existem no Museu de Lisboa. Jornal de Sciencias Mathematicas, Physicas e Naturaes 1 (3): 233-264.

Capello F.B. 1867b. Catálogo dos peixes de Portugal que existem no Museu de Lisboa. Jornal de Sciencias Mathematicas, Physicas e Naturaes 1 (4): 307-313.

Capello F. B. 1868. Catálogo dos peixes de Portugal que existem no Museu de Lisboa. Jornal de Sciencias Mathematicas, Physicas e Naturaes 2 (5): 51-63.

Capello F. B. 1869a. Catálogo dos peixes de Portugal que existem no Museu de Lisboa. Jornal de Sciencias Mathematicas, Physicas e Naturaes 2 (6): 131-153.

Capello F. B. 1869b. Appendice ao Catálogo dos peixes de Portugal que existem no Museu de Lisboa. Jornal de Sciencias Mathematicas, Physicas e Naturaes 2 (7): 223-228.

Capello F.B. 1873. Segundo apêndice ao catálogo dos peixes de Portugal. Jornal de Sciencias Mathematicas, Physicas e Naturaes 4 (16): 307-311.

Capello F.B. 1876. Terceiro apêndice ao catálogo dos peixes de Portugal. Jornal de Sciencias Mathematicas, Physicas e Naturaes 5 (19): 165-167.

Capello F.B. 1880. Catálogo dos peixes de Portugal. Memórias da Academia de Sciencias de Lisboa 6: 1-73.

Castro J.M.O. 1967. Nomenclatura Portuguesa do Pescado. Gabinete de Estudos das Pescas, Lisboa.

Coad J.O. \& Hüssy K. 2012. Boom in boarfish abundance: Insight from otolith analysis. In: ICES CM 2012/J:10: 1-24. International Council for the Exploration of the Sea (ICES).

Collette B.B., McDowell J.R. \& Graves J.E. 2006. Phylogeny of Recent Billfishes (Xiphioidei). Bulletin of Marine Science 79 (3): 455-468.

Compagno L.J.V. 1984a. FAO Species Catalogue. Sharks of the world. An annotated and illustrated catalogue of shark species known to date. Part 1- Hexanchiformes to Lamniformes. FAO Fisheries Synopsis 125: 1-249, Rome.

Compagno L.J.V. 1984b. FAO Species Catalogue. Sharks of the world. An annotated and illustrated catalogue of shark species known to date. Part 2- Carcharhiniformes. FAO Fisheries Synopsis 125: 251655 , Rome.

Compagno L.J.V. 1999. Checklist of living elasmobranchs. In: Hamlett, W.C. (ed.) Sharks, Skates, and Rays: The Biology of Elasmobranch Fishes: 471-498. John Hopkins University Press, Maryland.

Compagno L.J.V. 2003. Sharks. In: Carpenter K.E. (ed.) The living marine resources of the Western Central Atlantic. Volume 1. Introduction, molluscs, crustaceans, hagfishes, sharks, batoid fishes, and chimaeras. FAO species identification guide for fishery purposes and American Society of Ichthyologist and Herpetologists Special Publication No.5: 357-505. Food and Agriculture Organization, Rome.

Costa M.E. 2007. Chondrichthyes. In: Borges,T.C. (ed.) Biodiversidade nas Pescas do Algarve (Sul de Portugal). Impriluz, Lisboa. 
Costa F.O., Landi M., Martins R., Costa M.H., Costa M.E., Carneiro M., Alves M.J., Steinke D. \& Carvalho G.R. 2012. A ranking system for reference libraries of DNA Barcodes: Application to marine fish species from Portugal. PLoS ONE 7 (4): 1-9. http://dx.doi.org/10.1371/journal.pone.0035858

Costello M.J., Bouchet P., Emblow C.S. \& Legakis, A. 2006. European marine biodiversity inventory and taxonomic resources: state of the art and gaps in knowledge. Marine Ecology Progress Series 316: 257-268. http://dx.doi.org/10.3354/meps316257

Costello M.J., Coll M., Danovaro R., Halpin P., Ojaveer H. \& Miloslavich P. 2010. A census of marine biodiversity knowledge, resources, and future challenges. PLOS ONE 5 (8): 1-15. http://dx.doi. org/10.1371/journal.pone.0012110

Declaração de Rectificação n. ${ }^{\circ}$ 52/2006, de 18 de Agosto. Diário da República 159 I Série-A: 5849-5863.

Dyer B.S. \& Westneat M.W. 2010. Taxonomy and biogeography of the coastal fishes of Juan Fernández Archipelago and Desventuradas Islands, Chile. Revista de Biología Marina y Oceanografía, 45 (S1): 589-617. http://dx.doi.org/10.4067/S0718-19572010000400007

Ebert D.A. \& Stehmann M.F.W. 2013. Sharks, batoids, and chimaeras of the North Atlantic. FAO Species Catalogue for Fishery Purposes no. 7, Food and Agriculture Organization, Rome.

Ellis J.R., Engelhard G.H. \& Pinnegar J.K. 2008. Ecotypology of fishes in the eastern North Atlantic. RECLAIM, 26. Available from: http://www.climateandfish.eu

Eschmeyer W.N. (ed.) 2013. The Catalog of Fishes. California Academy of Sciences, San Francisco, California, U.S.A. Available from: http://researcharchive.calacademy.org/research/ichthyology/catalog/ fishcatmain.asp and http://collections.calacademy.org/ich/

Fowler H.W. 1936. The Marine Fishes of West Africa. Vol. LXX, Part I and Part II. The American Museum of Natural History, New York.

Froese R. \& Pauly D. 2012. Global Information System on Fishes - FISHBASE (version Dec. 2012). Available from: http://www.fishbase.org

Gomes J.M. \& Olim S. 2007. Actinopterygii. In: Borges, T.C. (ed.) Biodiversidade nas Pescas do Algarve (Sul de Portugal). Impriluz, Lisboa.

Gonçalves B.C. 1941. Colecção Oceanográfica de D. Carlos I - Peixes. Travaux da la Station de Biologie Maritime de Lisbonne 46: 1-108.

Gordo L.S. \& Cabral H.N. 2001. The fish assemblage structure of a hydrologically altered coastal lagoon: The Óbidos lagoon (Portugal). Hydrobiologia 459: 125-133.

Gunn J.S. 1990. A revision of selected genera of the Family Carangidae (Pisces) from Australian Waters. Records of the Australian Museum Suppl. 12: 1-77. http://dx.doi.org/10.3853/j.0812-7387.12.1990.92.

Hareide N.-R. \& Garnes G. 2001. The distribution and catches rates of deep water fish along the MidAtlantic Ridge from 43 to $61^{\circ} \mathrm{N}$. Fisheries Research 51:297-310.

Hernandez F. (ed.) 2013. EMODnet - European Marine Observation and Data Network. Vlaams Instituut Voor de Zee. Available from: http://bio.emodnet.eu/portal/index.php [accessed since 14 Feb. 2013].

Horta e Costa B. \& Gonçalves E.J. 2013. First occurrence of the Monrovia doctorfish Acanthurus monroviae (Perciformes: Acanthuridae) in European Atlantic waters. Marine Biodiversity Records 6 (20): 1-4. http://dx.doi.org/10.1017/S1755267213000055

Hureau J.C. \& Monod Th. 1979a. Check-list of the Fishes of the North-Eastern Atlantic and the Mediterranean (CLOFNAM I). United Nations Educational, Scientific and Cultural Organization, Paris. 
Hureau J.C. \& Monod Th. 1979b Check-list of the Fishes of the North-Eastern Atlantic and the Mediterranean (CLOFNAM II). United Nations Educational, Scientific and Cultural Organization, Paris.

Hureau J.C. \& Tortonese E. 1979. Carangidae. In: Hureau J.C. \& Monod Th. (1979a) Check-list of the Fishes of the North-Eastern Atlantic and the Mediterranean (CLOFNAM I): 373-384. United Nations Educational, Scientific and Cultural Organization, Paris.

Iglésias S.P., Toulhoat L. \& Sellos D.Y. 2010. Taxonomic confusion and market mislabelling of threatened skates: important consequences for their conservation status. Aquatic Conservation: Marine and Freshwater Ecosystems 20: 319-333. http://dx.doi.org/10.1002/aqc.1083

Iwamoto T. 2003. Family no 93 - Macrouridae. In: Smith J.L.B, Smith M.M. \& Heemstra P.C. (eds) Smith's Sea Fishes: 330-350. Struik, Cape Town.

Jonsson B. 2011. NOBANIS - Invasive Alien Species Fact Sheet - Oncorhynchus mykiss. Available from: http://www.nobanis.org/ Online Database of the European Network on Invasive Alien Species NOBANIS [accessed 10 Mar. 2013].

Koefoed E. 1932. Fishes from the sea-botton from the "Michael Sars" North Atlantic Deep-sea Expedition 1910. John Grieg (ed.), Bergen.

Kotlyar A.N. 2011. Revision of Genus Melamphaes (Melamphaidae). II. Multi-Raker Species: M. polylepis, M. falsidicus sp. nova, M. pachystomus sp. nova, M. macrocephalus, M. leprus. Journal of Ichthyology 51 (8): 569-580. http://dx.doi.org/10.1134/S0032945211050080

Kukuev E.I. 2002. Ichthyofauna research on underwater mountains within the North Atlantic Ridge and adjacent areas. 2002 Annual Science Conference, International Council for the Exploration of the Sea, Copenhagen, Denmark. International Council for the Exploration of the Sea - Committee Document CM2002/M: 05: 1-19.

Kukuev E.I., Parin N.V. \& Trunov I.A. 2012. Materials for the revision of the Family Caristiidae (Perciformes). 2. Manefishes from the East Atlantic (Redescription of Platyberyx opalescens Zugmayer and description of two new species Platyberyx mauli sp. n. and Caristius andriashevi sp. n.). Journal of Ichthyology 52 (3): 185-199. http://dx.doi.org/10.1134/S0032945212010080

Magalhães F. \& Rogado L. (eds) 1993. Livro Vermelho dos Vertebrados de Portugal, Vol.III - Peixes Marinhos e Estuarinos. Instituto da Conservação da Natureza, Lisboa.

Martins R., Costa F.O., Murta A.C., Carneiro M. \& Landi M. 2012. First record of Zenion hololepis (Zenionidae) in Portuguese continental waters: the northernmost occurrence in the eastern Atlantic. Marine Biodiversity Records 5: 1-3. http://dx.doi.org/10.1017/S1755267211000522

Maul G.E. 1948a. Lista sistemática dos peixes assinalados nos mares da Madeira. In: Noronha A.C. \& Sarmento A.A. (ed.) Vertebrados da Madeira: 135-159. Junta Geral do Distrito Autónomo do Funchal, Funchal.

Maul G.E. 1948b. Monografia dos Peixes do Museu Municipal do Funchal. Ordem Isospondyli. Boletim do Museu Municipal do Funchal 3 (5): 5-41.

Maul G.E. 1948c. Quatro peixes novos dos mares da Madeira. Boletim do Museu Municipal do Funchal 3 (6): 41-55.

Maul G.E. 1949. Monografia dos Peixes do Museu Municipal do Funchal. Ordem Isospondyli, Conclusão. Boletim do Museu Municipal do Funchal 4 (9): 1-20.

Maul G.E. 1951a. Monografia dos Peixes do Museu Municipal do Funchal. Família Macrouridae e Merlucciidae. Boletim do Museu Municipal do Funchal 5 (12): 5-55.

Maul G.E. 1951b. Nota sobra as duas espécies de género Neoscopelus. Boletim do Museu Municipal do Funchal 5 (13): 56-63. 
Maul G.E. 1952a. Monografia dos Peixes do Museu Municipal do Funchal: Famílias Gadidae e Bregmacerotidae. Boletim do Museu Municipal do Funchal 6 (15): 5-51.

Maul G.E. 1952b. Monografia dos Peixes do Museu Municipal do Funchal. Additions to previously revised families. Boletim do Museu Municipal do Funchal 6 (16): 51-62.

Maul G.E. 1954a. Monografia dos Peixes do Museu Municipal do Funchal. Ordem Berycomorphi. Boletim do Museu Municipal do Funchal 7 (17): 1-41.

Maul G.E. 1954b. Monografia dos Peixes do Museu Municipal do Funchal. Additions to previously revised families. Boletim do Museu Municipal do Funchal 7 (18): 41-63.

Maul G.E. 1955. Monografia dos Peixes do Museu Municipal do Funchal. Ordem Heteromi. Boletim do Museu Municipal do Funchal 8 (20): 5-19.

Maul G.E. 1956a. Monografia dos Peixes do Museu Municipal do Funchal. Ordem Discocephali. Boletim do Museu Municipal do Funchal 9 (23): 5-75.

Maul G.E. 1956b. Monografia dos Peixes do Museu Municipal do Funchal. Additions to previously revised Orders or Families of Fishes of the Museu Municipal do Funchal (Stomiatidae, Astronesthidae, Paralepididae). Boletim do Museu Municipal do Funchal 9 (24): 75-96.

Maul G.E. 1957. Further addition to the previously revised family Searsidae. Boletim do Museu Municipal do Funchal 10 (25): 1-21.

Maul G.E. 1959. On a specimen of Bathylaco nigricans Good and Bean taken from the stomach of Aphanopus carbo. Museu Municipal do Funchal, Bocagiana 12 (4): 1-8.

Maul G.E. 1961. The ceratioid fishes in the collection of the Museu Municipal do Funchal (Melanocetidae, Himantolophidae, Oneirodidae, Linophrynidae). Boletim do Museu Municipal do Funchal 14 (50): 87159.

Maul G.E. 1962a. On a small collection of ceratioid fishes from off Dakar and two recently acquired specimens from a stomachs of Aphanopus carbo taken in Madeira (Melanocetidae, Himantolophidae, Diceratiidae, Oneirodidae, Ceratiidae). Boletim do Museu Municipal do Funchal 16 (54): 5-27.

Maul G.E. 1962b. Report on the fishes taken in Madeira and Canarian waters during the summer-autumn cruises of the "Discovery II" 1959 and 1961. I Ceratioid fishes (Melanocetidae, Himantolophidae, Oneirodidae, Gigantactinidae, Linophrynidae). Boletim do Museu Municipal do Funchal 16 (56): 33-46.

Maul G.E. 1965. On a new genus and species of Paralepidid from Madeira. Boletim do Museu Municipal do Funchal 19 (81): 55-61-

Maul G.E. 1971a. Report on the fishes taken in Madeira and Canarian waters during the summerautumn cruises of the "Discovery II" 1959 and 1961. III Order Iniomi I on a toothless, sexually mature Anotopterus. Museu Municipal do Funchal, Bocagiana 28: 1-15.

Maul G.E. 1971b. On a new Goby of the Genus Lesueurigobius from off the Atlantic coast of Marocco and Madeira (Percomorphi, Gobioidae, Gobiidae). Museu Municipal do Funchal, Bocagiana 29: 1-7.

Maul G.E. 1972. On a new species of eel of the genus Gnathophis (Apodes, Congridae) from the Meteor Seamount. Museu Municipal do Funchal, Bocagiana 31: 1-7.

Melo M.R.S. 2008. The genus Kali Lloyd (Chiasmodontidae: Teleostei) with description of new two species, and the revalidation of K. kerberti Weber. Zootaxa 1747: 1-33.

Menezes G.M., Rosa A., Melo O. \& Porteiro F.M. 2012. Annotated list of demersal fishes occurring at Sedlo seamount, Azores North-east Atlantic Central Ocean. Journal of Fish Biology 81: 1003-1018. http://dx.doi.org/10.1111/j.1095-8649.2012.03355.x 
Moser H.G. \& Charter S.R. 1996. Notacanthidae: spinyeels. In: Moser H.G. (ed.) The early stages of fishes in the California Current Region: 82-85. California Cooperative Oceanic Fisheries Investigations (CalCOFI) Atlas 33, La Jolla (California, USA).

Moura R.L. \& Castro R.M.C. 2002. Revision of Atlantic sharpnose pufferfishes (Tetraodontiformes: Tetraodontidae: Canthigaster), with description of three new species. Proceedings of the Biological Society of Washington 115: 32-50.

Muñoz-Chapuli R. \& Ramo F. 1989. Review of the Centrophorus sharks (Elasmobranchii, Squalidae) of the Eastern-Atlantic. Cybium 13 (1): 65-81.

Murray J. 1895. Report on the Scientific Results of the Voyage of HMS "Challenger". A Summary of the Scientific Results, Part. 2. Her Majesty's Stationery Office, London, 797-1608.

Murray J. \& Hjort J. 1912. Depths of the Ocean. Macmillan and Co., London. http://dx.doi.org/10.5962/ bhl.title. 6874

Naylor G.J.P., Caira J.N., Jensen K., Rosana K.A.M., Straube N. \& Lakner C. 2012. Elasmobranch phylogeny: A mitochondrial estimate based on 595 species. In: Carrier J.C., Musick J.A. \& Heithaus M.R. (eds) The Biology of Sharks and Their Relatives: 31-56. Chemical Rubber Company Press, New York.

Nelson J.S., 2006. Fishes of the World (Fourth Edition). John Wiley \& Sons, Inc., New Jersey.

Nichols, J.T. 1938. Notes on Carangin Fishes (III - On Caranx sexfasciatus Quoy \& Gaimard). American Museum Novitates 998: 1-6.

Nielsen J.G. \& Møller P.R. 2008. New and rare deep-sea ophidiiform fishes from the Solomon Sea caught by the Danish Galathea 3 Expedition. Steenstrupia 30 (1): 21-46.

Nobre A. 1935. Fauna Marinha de Portugal. I. Vertebrados (Mamiferos, Répteis e Peixes). Companhia Editora do Minho, Barcelos.

Nolf D. 2013. The Diversity of Fish Otoliths, Past and Present. Royal Belgian Institute of Natural Sciences, Brussels.

Osório B. 1888. Aditamento ao catálogo dos peixes de Portugal. Jornal de Sciencias Mathematicas, Physicas e Naturaes - $1^{\text {a }}$ Série, 12: 1-19.

Osório B. 1895. Segundo apêndice ao "Catálogo dos Peixes de Portugal" de Felix Capello. Jornal de Sciencias Mathematicas, Physicas e Naturaes 3: 254-269.

Osório B. 1896. Peixes de Matozinhos. Terceiro apêndice ao catálogo dos peixes de Portugal de F. Capello. Jornal de Sciencias Mathematicas, Physicas e Naturaes 4: 131-159.

Osório B. 1905. Notícia de uma espécie a juntar ao catálogo dos peixes de Portugal de F. Capello. Jornal de Sciencias Mathematicas, Physicas e Naturaes 7: 151-152.

Osório B. 1909. Contribuição para o conhecimento da fauna bathypelagica visinhas das costas de Portugal, Lisboa.

Parin N.V. \& Borodulina O.D. 1990. Survey of the genus Polymetme (Photichthyidae) with a description of two new species. Journal of Ichthyology 30 (6): 108-121.

Parin N.V. \& Borodulina O.D. 2000. Redescriptions and new data on the distribution of six rare and poorly known species of the mesopelagic fish genus Astronesthes (Astronesthidae). Journal of Ichthyology 40, Suppl. 1: 15-30.

Patterson D.J., Cooper J., Kirk P.M., Pyle R.L. \& Remsen D.P. (eds) 2010. Global Names Index. Available from: http://gni.globalnames.org/name_strings 
Pietsch T.W. 2005. New species of the ceratoniod anglerfish genus Lasiognathus Regan (Lophiiformes: Thaumatichthyidae) from the Eastern North Atlantic off Madeira. Copeia 2005 (1): 77-81.

Portaria n. ${ }^{\circ}$ 587/2006, de 22 de Junho - List of Authorized Commercial Names for Fishery and Aquaculture Products - Annex I. Diário da República 119 I, Série-B 4: 4421-4444.

Porteiro F.M. 2005 Biogeography and biodiversity of Stomiid fish in the North Atlantic. Ph.D. thesis, University of Liverpool, $U K$.

Porteiro F.M. 2009. A importância das campanhas oceanográficas do Príncipe Albert I do Mónaco para o conhecimento do Mar dos Açores. Boletim do Núcleo Cultural da Horta 18: 189-219.

Porteiro F.M., Menezes G.M., Afonso P., Monteiro J.G. \& Santos R.S. 2010. Marine fish (Chondrichthyes, Actinopterygii). In: Borges P.A.V., Costa A., Cunha R., Gabriel R., Gonçalves V., Martins A.F., Melo I., Parente M., Raposeiro P., Rodrigues P., Santos R.S., Silva L., Vieira P., \& Vieira V. A list of the terrestrial and marine biota from the Azores: 325-345. Principia, Cascais.

Post A. 1987. Pelagic transects of FRVs "Walther Herwig" and "Anton Dohrn" in the Atlantic Ocean 1966 to 1986. Mitteilungen aus dem Institut für Seefischerei durch BfaFi der Bundesforschungsanstalt für Fischerei 42: 1-68.

Post A. \& Quéro J.-C. 1991. Distribution et taxonomie des Howella (Perciformes, Percichthyidae) de l'Atlantique. Cybium 15 (2): 111-128.

Prokofiev A.M. 2006. Morphology of Howella sherborni (Perciformes, Percoidei) with comparative data on the families Acropomatidae, Epigonidae and Apogonidae. Kompanija Sputnik, Moscow.

Prokofiev A.M. 2007a. Osteology and some other morphological characters of Howella sherborni, with a discussion of the systematic position of the genus (Perciformes, Percoidei). Journal of Ichthyology 27 (6): 413-426. http://dx.doi.org/10.1134/S003294520706001X

Prokofiev A.M. 2007b. The osteology of Bathysphyraenops simplex and the diagnosis of the Howellidae (Perciformes: Percoidei) family. Journal of Ichthyology 47 (8): 556-578. http://dx.doi.org/10.1134/S0032945207080036

Quéro J.-C. 1982. Zeiformes. In: Maurin C. \& Quéro J.-C. (eds) Poisson des côtes nord-ouest africaines (Campagnes de la 'Thalassa' 1962, 1968, 1971 et 1073). Revue des Travaux de l'Institut des Pêches Maritimes 45: 5-71. Paris.

Quéro J.-C., Du Buit M.-H. \& Vayne J.-J. 1998. Les observations de poissons tropicaux et le réchauffement des eaux dans l'Atlantique européen. Oceanologica Acta 21 (2): 345-351. http://dx.doi.org/10.1016/S0399-1784(98)80021-2

Quéro J.-C., Porché P. \& Vayne J.-J. 2003. Guide des Poissons de l'Atlantique Européen. Delachaux et Niestlé, Paris.

Rannou M., Nielsen J.G. \& Hureau J-C. 1974. Note sur quelques Aphyonidae de l'Atlantique Nord (Téléostéens, Ophidioidei). Bulletin du Muséum National d'Histoire Naturelle 171 (247): 1249-1257.

Ratnasingham S. \& Hebert P.D.N. 2007. BOLD: The Barcode of Life Data System (http://www. barcodinglife.org). Molecular Ecology Notes 7: 355-364. http://dx.doi.org/10.1111/j.14718286.2007.01678.x

Robalo J.I., Santos C.S., Cabral H., Castilho R. \& Almada V.C. 2009. Genetic evidence fails to discriminate between Macroramphosus gracilis Lowe 1839 and Macroramphosus scolopax Linnaeus 1758 in Portuguese waters. Marine Biology 156: 1733-1737. http://dx.doi.org/10.1007/s00227-009$\underline{1197-y}$ 
Rogado L., Alexandrino P., Almeida P.R., Alves J., Bochechas J., Cortes R., Domingos I., Filipe F., Madeira J. \& Magalhães F. 2005. Peixes. In: Cabral M.J., Almeida J., Almeida P.R., Dellingar T., Ferrand de Almeida N., Oliveira M.E., Palmeirim J.M., Queiroz A., Rogado L. \& Santos-Reis M. (eds) Livro Vermelho dos Vertebrados de Portugal: 40-43. Instituto da Conservação da Natureza, Lisboa.

Sampaio A. S. 1904. Memoria sobre a Ilha Terceira. Imprensa Municipal, Angra do Heroísmo.

Sanches J.G. 1986. Nomenclatura e Diagnose dos Principais Peixes Marinhos de Portugal (Ciclóstomos, Seláceos e Holocéfalos). Publicações Avulsas do Instituto Nacional de Investigação das Pescas 9, INIP, Lisboa.

Sanches J.G. 1989. Nomenclatura Portuguesa de Organismos Aquáticos (Proposta para Normalização Estatística). Publicações Avulsas do Instituto Nacional de Investigação das Pescas 14, INIP, Lisboa.

Santos R.S., Porteiro F.M. \& Barreiros J.P. 1997. Marine fishes of the Azores: annotated checklist and bibliography. Arquipélago - Life and Marine Sciences. Suppl. 1: 1-244.

Seabra A.F. 1911. Catalogue systématique des Vertébrés du Portugal. V. Poissons. Bulletin de la Société Portugaise des Sciences Naturelles 53: 129-224.

Shcherbachev Y.N., Kukuev E.I. \& Shlibanov V.I. 1985. Composition of the benthic and demersal ichthyocenoses of the submarine mountains in the southern part of the North Atlantic Range. Jounal of Ichthyology 25: 110-125.

Smith D.G. 1989. Various eel families. In: Böhlke E.B. (ed.) Orders Anguilliformes and Saccopharyngiformes. Memoirs of the Sears Foundation of Marine Research 1(9), Yale Peabody Museum of Natural History, Connecticut.

Smith D.G. 2012. A checklist of the moray eels of the world (Teleostei: Anguilliformes: Muraenidae). Zootaxa 3474: 1-64.

Southward A.J., Langmead O., Hardman-Mountford N.J., Aiken J., Boalch G.T., Dando P.R., Genner M.J., Joint I., Kendall M., Halliday N.C., Harris R.P., Leaper R., Mieszkowska N., Pingree R.D., Richardson A.J., Sims D.W., Smith T., Walne A.W. \& Hawkins S.J. 2005. Long-term oceanographic and ecological research in the western English Channel. Advances in Marine Biology 47: 1-105. http://dx.doi.org/10.1016/S0065-2881(04)47001-1

Stefansdottir L., Solmundsson J., Marteinsdottir G., Kristinsson K.N. \& Jonasson J.P. 2010. Groundfish species diversity and assemblage structure in Icelandic waters during recent years of warming. Fisheries Oceanography 19: 42-62. http://dx.doi.org/10.1111/j.1365-2419.2009.00527.x

Stein D.L. 2005. Descriptions of four new species, redescription of Paraliparis membranaceus, and additional data on species of the fish family Liparidae (Pisces, Scorpaeniformes) from the west coast of South America and the Indian Ocean. Zootaxa 1019: 1-25.

Stein D.L. \& Able K.J. 1986. Liparididae. In: Whitehead P.J.P., Bauchot M.-L., Hureau J.-C., Nielsen J. $\&$ Tortonese E. (eds) Fishes of the North-eastern Atlantic and Mediterranean (FNAM III). UNESCO, Paris, 1275-1283.

Stein D.L., Melendez C.R. \& Kong U.I. 1991. A review of Chilean snailfishes (Liparididae, Scorpaeniformes) with descriptions of a new genus and three new species. Copeia 1991 (2): 358-373.

Swinney G.N. 1994. Comments on the Atlantic species of the genus Evermannella (Scopelomorpha, Aulopiformes, Evermannellidae) with a re-evaluation of the status of Evermanella melanoderma. Journal of Fish Biology 44: 809-819. http://dx.doi.org/10.1111/j.1095-8649.1994.tb01257.x

Vaillant L.L. \& Milne-Edwards A. 1888. Expeditions scientifiques du Travailleur et du Talisman pendant les années 1880, 1881, 1882, 1883: Poissons. G. Masson, Paris. 
Vandelli D. 1771. Hortus Olisiponensis Exhibens Plantas Exoticas Horti Regii Specimenque Historiae Naturalis Lusitaniae Cum novis generibus et Specibus. Manuscript. Available from: http://purl. $\mathrm{pt} / 15104 / 1 / \mathrm{P} 7 . \mathrm{html}$

Vandelli D. 1797. Flora et fauna Lusitanicae specimen. Memórias da Academia de Sciencias de Lisboa 1.

Vanden Berghe E., Van Guelpen L. \& Pohle G. 2005. NARMS - North Atlantic Register for Marine Species. Available from: http://www.vliz.be/vmdedata/narms/

Van der Land J., Costello M.J., Zavodnik D., Santos R.S., Porteiro F.M., Bailly N., Eschmeyer W.N. \& Froese R. 2001. Pisces, In: Costello M.J., Emblow C.S. \& White R.J. (eds) European Register of Marine Species: A Checklist of the Marine Species in Europe and A Bibliography of Guides to Their Identification 357-374. Collection Patrimoines Naturels 50, Paris.

Vieira R.P., Christiansen B., Christiansen S. \& Gonçalves J.M.S. 2012. First record of the deep-water whalefish Cetichthys indagator (Actinopterygii: Cetomimidae) in the North Atlantic Ocean. Journal of Fish Biology 81: 1133-1137. http://dx.doi.org/10.1111/j.1095-8649.2012.03378.x

Vierros M., Cresswell I., Briones E.E., Rice J. \& Ardron J. 2009. Global Open Oceans and Deep Seabed (GOODS) - Biogeographic Classification. United Nations Educational, Scientific and Cultural Organization - Intergovernmental Oceanographic Commission, IOC Technical Series 84, Paris.

Wirtz P. 1998. Twelve invertebrate and eight fish species new to the marine fauna of Madeira, and a discussion of the zoogeography of the area. Helgoländer wissenschaftliche Meeresuntersuchungen 52: 197-207.

Wirtz P., Fricke R. \& Biscoito M.J. 2008. The coastal fishes of Madeira Island - new records and an annotated check-list. Zootaxa 1715: 1-26.

WoRMS Editorial Board 2013. World Register of Marine Species. Available from http://www. marinespecies.org/ at VLIZ.

Zahuranec B.J. 2000. Zoogeography and Systematics of the Lanternfishes of the Genus Nannobrachium (Myctophidae: Lampanyctini). Smithsonian Contributions to Zoology 607. Smithsonian Institution Press, Washington D.C.

Manuscript received: 13 May 2013

Manuscript accepted: 8 November 2013

Published on: 6 February 2014

Topic editor: Rudy Jocqué

Desk editor: Kristiaan Hoedemakers

Printed versions of all papers are also deposited in the libraries of the institutes that are members of the EJT consortium: Muséum National d'Histoire Naturelle, Paris, France; National Botanic Garden of Belgium, Meise, Belgium; Royal Museum for Central Africa, Tervuren, Belgium; Natural History Museum, London, United Kingdom; Royal Belgian Institute of Natural Sciences, Brussels, Belgium; Natural History Museum of Denmark, Copenhagen, Denmark. 